\title{
Decoupled Scheme for Time-Dependent Natural Convection Problem II: Time Semidiscreteness
}

\author{
Tong Zhang and ZhenZhen Tao \\ School of Mathematics \& Information Science, Henan Polytechnic University, Jiaozuo 454003, China \\ Correspondence should be addressed to Tong Zhang; zhangtong0616@163.com
}

Received 28 May 2014; Revised 11 November 2014; Accepted 20 November 2014; Published 21 December 2014

Academic Editor: Jitao Sun

Copyright (C) 2014 T. Zhang and Z. Tao. This is an open access article distributed under the Creative Commons Attribution License, which permits unrestricted use, distribution, and reproduction in any medium, provided the original work is properly cited.

We study the numerical methods for time-dependent natural convection problem that models coupled fluid flow and temperature field. A coupled numerical scheme is analyzed for the considered problem based on the backward Euler scheme; stability and the corresponding optimal error estimates are presented. Furthermore, a decoupled numerical scheme is proposed by decoupling the nonlinear terms via temporal extrapolation; optimal error estimates are established. Finally, some numerical results are provided to verify the performances of the developed algorithms. Compared with the coupled numerical scheme, the decoupled algorithm not only keeps good accuracy but also saves a lot of computational cost. Both theoretical analysis and numerical experiments show the efficiency and effectiveness of the decoupled method for time-dependent natural convection problem.

\section{Introduction}

In this paper, we consider the following time-dependent natural convection problem in $\mathbb{R}^{2}$ whose coupled equations governing viscous incompressible flow and heat transfer for the incompressible fluid are Boussinesq system:

$$
\begin{gathered}
u_{t}-v \Delta u+(u \cdot \nabla) u+\nabla p=-k v^{2} j \theta+f, \quad \text { in } \Omega \times(0, T], \\
\operatorname{div} u=0, \quad \text { in } \Omega \times(0, T], \\
\theta_{t}-\lambda v \Delta \theta+u \cdot \nabla \theta=g, \quad \text { in } \Omega \times(0, T], \\
u=0, \quad \theta=0, \quad \text { on } \partial \Omega \times(0, T], \\
u(x, 0)=u_{0}, \quad \theta(x, 0)=\theta_{0}, \quad \text { on } \Omega \times\{0\},
\end{gathered}
$$

where $\Omega$ is a bounded convex polygonal domain, $u=\left(\begin{array}{l}u_{1} \\ u_{2}\end{array}\right)$ is the fluid velocity, $p$ is the pressure, $\theta$ is the temperature, $v>0$ is the viscosity, $k$ is the Grashof number, $\lambda=\operatorname{Pr}^{-1}, \operatorname{Pr}$ is the Prandtl number, $j=\left(\begin{array}{l}1 \\ 0\end{array}\right)$ is the vector of gravitational acceleration, $T>0$ is the final time, and $f$ and $g$ are forcing functions.

The time-dependent natural convection problem (1) is an important system with dissipative nonlinear terms in atmospheric dynamics (see [1]). Since this system not only contains the velocity and pressure but also includes the temperature filed, finding the numerical solution of problem (1) becomes a difficult task. For the study of problem (1), many researchers have developed several kinds of efficient numerical schemes, for example, the standard Galerkin finite element method (FEM) [2], the projection-based stabilized MFEM [3, 4], and the references therein. Here, we need to point out that all these numerical schemes for problem (1) are coupled. It means that we need to find the variables $u, p$, and $\theta$ of (1) simultaneously; as a consequence, a large nonlinear algebra system is formed. In general, it is expensive to find the numerical solutions of the coupled nonlinear system directly in standard Galerkin FEM.

The decoupled algorithm is an efficient numerical scheme for the multivarious problems. There are many advantages for the decoupled method. For example, it allows us to search the algorithm components flexibly and conveniently in terms of physical, mathematical, and numerical properties for each variable. It is suitable for today's computing environment because it can efficiently and effectively exploit the existing computing resources, including both hardware and software. The decoupled method can be used in parallel in the conventional sense; other appealing reasons were discussed in [5]. The decoupled algorithm has been successfully applied to the 
multidomain problem, for example, $\mathrm{Mu}$ and his coworkers $[5,6]$ for the Stokes-Darcy problem, Layton and his coauthors $[7,8]$ for the groundwater-surface water flows, and Zhang et al. $[9,10]$ for coupling fluid flow with porous media flow. In view of the efficiency of the decoupled scheme, we try to extend it to solve the time-dependent natural convection problem (1). The decoupled time semidiscrete scheme is closely related to the usual temporal extrapolation method $[8,11]$. Thanks to the decoupled scheme, we can decouple the complex and nonlinear problem into two small linear subproblems, and the coefficient matrix of each subproblem is symmetric; therefore, these subproblems can be solved easier than the origin problem.

In this paper we establish the optimal error estimates for velocity, pressure, and temperature for problem (1) in both coupled and decoupled numerical schemes. Firstly, problem (1) is discrete in standard Galerkin finite element formulation based on the backward Euler scheme; then a large and nonlinear algebraic system is formed. Secondly, in order to simplify the computation, we adopt the decoupled and linearized algorithm to solve problem (1). Namely, the temporal extrapolation technique is used to treat the nonlinear terms, and then problem (1) is split into two subproblems, each subproblem can be solved easier than the origin problem. Furthermore, compared with the coupled scheme, these two subproblems which were obtained by using the decoupled method can be solved in parallel.

Under the conditions of (A1)-(A6) which are presented in Section 2, the numerical solution $\left(u^{d, n}, p^{d, n}, \theta^{d, n}\right)$ of the decoupled method at the time level $t_{n}=n \Delta t$ satisfies the following error estimates for all time step $\Delta t>0$ :

$$
\begin{aligned}
& \left\|\nabla\left(u\left(t_{n}\right)-u^{d, n}\right)\right\|_{0}+\left\|p\left(t_{n}\right)-p^{d, n}\right\|_{0}+\left\|\nabla\left(\theta\left(t_{n}\right)-\theta^{d, n}\right)\right\|_{0} \\
& \quad \leq C \Delta t
\end{aligned}
$$

where $C$ is a generic constant depending on the data $(\Omega, \nu, k$, $\left.\lambda, u_{0}, \theta_{0}, f_{\infty}, g_{\infty}, T\right)$ independent of $\Delta t$ and it may stand for different values at different places:

$$
\begin{array}{ll}
f_{\infty}=\sup _{t \geq 0}\left\{|f(t)|+\left|f_{t}(t)\right|\right\}, \quad f_{t}=\frac{d f}{d t}, \\
g_{\infty}=\sup _{t \geq 0}\left\{|g(t)|+\left|g_{t}(t)\right|\right\}, \quad g_{t}=\frac{d g}{d t} .
\end{array}
$$

Under the conditions of (A1)-(A6), the numerical solution $\left(u^{n}, p^{n}, \theta^{n}\right)$ of the coupled method satisfies the following error estimates for all $\Delta t>0$ :

$$
\begin{aligned}
& \left\|\nabla\left(u\left(t_{n}\right)-u^{n}\right)\right\|_{0}+\left\|p\left(t_{n}\right)-p^{n}\right\|_{0}+\left\|\nabla\left(\theta\left(t_{n}\right)-\theta^{n}\right)\right\|_{0} \\
& \quad \leq C \Delta t
\end{aligned}
$$

From (2) and (4), we can see that the coupled and decoupled algorithms have the same order of approximation. While there are only two small linear subproblems that need to be solved in the decoupled algorithm, a lot of memory and computational work can be saved.

The outline of this paper is as follows. We recall some basic notations and results for problem (1) in Section 2. Section 3 is devoted to present the coupled and decoupled algorithms for problem (1). Stabilities of both the coupled and decoupled schemes are established in Section 4. Optimal error estimates of numerical solutions in both the coupled and decoupled numerical schemes are presented in Sections 5 and 6 , respectively. Finally, we provide some numerical results to verify the efficiency and effectiveness of the decoupled algorithm for time-dependent natural convection problem.

\section{Preliminaries}

In this section, we will construct the variable formulation for problem (1) and develop some necessary assumptions which will be frequently used in this paper. To fix the idea, we set

$$
\begin{gathered}
X=H_{0}^{1}(\Omega)^{2}, \quad W=H_{0}^{1}(\Omega), \quad Y=L^{2}(\Omega)^{2}, \\
Z=L^{2}(\Omega), \quad M=L_{0}^{2}(\Omega)=\left\{\varphi \in L^{2}(\Omega): \int_{\Omega} \varphi d x=0\right\} .
\end{gathered}
$$

In this paper, we adopt $(\cdot, \cdot)$ and $\|\cdot\|_{0}$ to denote the inner product and norm in $Z$ or $Y$. The spaces $W$ and $X$ are equipped with the usual scalar product $(\nabla \cdot, \nabla \cdot)$ and norm $\|\nabla \cdot\|_{0}$. We define the continuous bilinear forms $a(\cdot, \cdot), d(\cdot, \cdot)$, and $\bar{a}(\cdot, \cdot)$, respectively, by

$$
\begin{gathered}
a(u, v)=v(\nabla u, \nabla v), \quad d(v, q)=(q, \operatorname{div} v), \\
\bar{a}(\theta, \psi)=\lambda v(\nabla \theta, \nabla \psi)
\end{gathered}
$$

for all $u, v \in X, q \in M$, and $\theta, \psi \in W$.

Next, we introduce the closed subset $V$ of $X$ given by

$$
\begin{aligned}
V & =\{v \in X: d(v, q)=0, \forall q \in M\} \\
& =\{v \in X, \nabla \cdot v=0 \text { in } \Omega\}
\end{aligned}
$$

and denote the $H$ to be the closed subset of $Y($ see $[11,12])$ :

$$
H=\left\{v \in Y, \nabla \cdot v=0,\left.v \cdot n\right|_{\partial \Omega}=0\right\}
$$

We denote by $A$ the unbounded linear operator on $Y$ or $Z$ given by $A u=-\Delta u$ or $A \theta=-\Delta \theta$ and assume that the domain of $A$ is given by (see $[13,14]$ )

$$
D(A)=H^{2}(\Omega)^{2} \cap X \quad \text { or } \quad E(A)=H^{2}(\Omega) \cap W .
$$

For instance, (9) holds if $\Gamma$ is of class $C^{2}$ or if $\Omega$ is a convex plane polygonal domain. 
Moreover, the trilinear terms for all $u, v, w \in X$ and $\theta, \psi \in$ $W$ can be defined as follows:

$$
\begin{aligned}
b(u, v, w) & =((u \cdot \nabla) v, w)+\frac{1}{2}((\operatorname{div} u) v, w) \\
& =\frac{1}{2}((u \cdot \nabla) v, w)-\frac{1}{2}((u \cdot \nabla) w, v), \\
\bar{b}(u, \theta, \psi) & =((u \cdot \nabla) \theta, \psi)+\frac{1}{2}((\operatorname{div} u) \theta, \psi) \\
& =\frac{1}{2}((u \cdot \nabla) \theta, \psi)-\frac{1}{2}((u \cdot \nabla) \psi, \theta) .
\end{aligned}
$$

With above notations, for a given $f \in L^{\infty}(0, T ; Y)$ with $u_{0} \in D(A) \cap V$ and $g \in L^{\infty}(0, T ; Z)$ with $\theta_{0} \in E(A)$, the variational formulation of problem (1) reads as follows: for all $(v, q, \psi) \in X \times M \times W$, find a pair $(u, p, \theta)$ with

$$
\begin{aligned}
& u \in L^{\infty}\left(R^{+} ; X\right) \cap L^{2}(0, T ; V), \quad u_{t} \in L^{2}\left(0, T ; V^{\prime}\right), \\
& \theta_{t} \in L^{2}\left(0, T ; W^{\prime}\right), \quad p \in L^{2}(0, T ; M), \\
& \theta \in L^{\infty}\left(R^{+} ; W\right) \cap L^{2}(0, T ; Z),
\end{aligned}
$$

$$
\forall T>0
$$

such that

$$
\begin{gathered}
\left(u_{t}, v\right)+a(u, v)-d(v, p)+b(u, u, v) \\
=-k v^{2}(j \theta, v)+(f, v), \\
d(u, q)=0, \\
\left(\theta_{t}, \psi\right)+\bar{a}(\theta, \psi)+\bar{b}(u, \theta, \psi)=(g, \psi), \\
u(x, 0)=u_{0}, \quad \theta(x, 0)=\theta_{0} .
\end{gathered}
$$

We make the regularity assumptions on the following problems [15-17].

(A1) Assume that $\Omega$ is smooth such that there exists a unique solution $(v, q) \in X \times M$ of the following Stokes problem:

$$
-\Delta v+\nabla q=u, \quad \nabla \cdot v=0, \quad \text { in } \Omega,\left.v\right|_{\partial \Omega}=0
$$

for any prescribed $u \in H$. Furthermore, the solution $v=A^{-1} u$ satisfies

$$
\|v\|_{s}=\left\|A^{-1} u\right\|_{s} \leq C_{1}\|u\|_{s-2}, \quad s=1,2 .
$$

Form (13), it follows that $\left(A^{-1} u, u\right)=\left\|\nabla A^{-1} u\right\|_{0}$ and

$$
\|v\|_{V^{\prime}}^{2}=\left(A^{-1} v, v\right), \quad \forall v \in H
$$

where $V^{\prime}$ is the dual space of $V$ and $C_{i}(i=1,2, \ldots)$ denotes a positive constant depending on $\Omega$.

(A2) Assume that $\Omega$ is smooth such that there exists a unique solution $\psi \in W$ of the following elliptic problem:

$$
-\Delta \psi=\theta, \quad \text { in } \Omega,\left.\psi\right|_{\partial \Omega}=0
$$

for any prescribed $\theta \in Z$. Furthermore, the solution $\psi=A^{-1} \theta$ satisfies

$$
\|\psi\|_{s}=\left\|A^{-1} \theta\right\|_{s} \leq C_{2}\|\theta\|_{s-2}, \quad s=1,2 .
$$

Form (16), it follows that $\left(A^{-1} \theta, \theta\right)=\left\|\nabla A^{-1} \theta\right\|_{0}$ and

$$
\|\psi\|_{W^{\prime}}^{2}=\left(A^{-1} \theta, \theta\right), \quad \forall \psi \in Z,
$$

where $W^{\prime}$ is the dual space of $W$

Assume that $f \in L^{2}\left(0, T ; X^{\prime}\right), g \in L^{2}\left(0, T ; W^{\prime}\right)$, and $u_{0} \in V, \theta_{0} \in W$. Problem (12) has at least one solution $(u, \theta)$ satisfying $u \in L^{\infty}(0, T ; \Omega) \cap L^{2}(0, T ; V)$ and $\theta \epsilon$ $L^{\infty}(0, T ; \Omega) \cap L^{2}(0, T ; W)$. Uniqueness and regularity of the solution $(u, p, \theta)$ can also be proved by strengthening the assumptions on the data. In particular, we assume that $u, p$, and $\theta$ satisfy the following

(A3) $u \in L^{\infty}\left(0, T ; H^{2}(\Omega)^{2}\right), \nabla p \in L^{\infty}(0, T ; Y), \theta \in$ $L^{\infty}\left(0, T ; H^{2}(\Omega)\right)$.

(A4) $u_{t} \in L^{2}(0, T ; X), \theta_{t} \in L^{2}(0, T ; W)$.

(A5) $\sqrt{t} u_{t t} \in L^{2}(0, T ; Y), \sqrt{t} \theta_{t t} \in L^{2}\left(0, T ; L^{2}\right)$.

(A6) $u_{t t} \in L^{2}\left(0, T ; V^{\prime}\right), \theta_{t t} \in L^{2}\left(0, T ; W^{\prime}\right)$.

(A7) $u_{t t t} \in L^{2}\left(0, T ; V^{\prime}\right), \theta_{t t t} \in L^{2}\left(0, T ; W^{\prime}\right)$.

Note that all such assumptions are feasible. For example, (A3) and (A4) can be proved with assumptions $u_{0} \in H^{2}(\Omega)^{2} \cap$ $V, \theta_{0} \in H^{2}(\Omega), f \in L^{\infty}(0, T ; H), f_{t} \in L^{2}(0, T ; H)$ and $g \in L^{\infty}(0, T ; Z), g_{t} \in L^{2}(0, T ; Z)$. When $\Omega$ is of class of $C^{2}$ or is a convex polygon, (A5) holds by $[11,12]$. Furthermore, (A6) holds by Shen in $[18,19]$ when he adds some nonlocal compatibility conditions at $t=0$. A review of regularity results for Navier-Stokes equations and applications to error estimates for Euler-type scheme can be found in [20], where the proof of (A7) was given.

We recall the following discrete Gronwall lemma, which can be found in $[11,18]$.

Lemma 1. Let $C_{0}$ and $a_{k}, b_{k}, c_{k}, d_{k}$, for integers $k \geq 0$, be nonnegative numbers such that

$$
a_{n}+\Delta t \sum_{k=0}^{n} b_{k} \leq \Delta t \sum_{k=0}^{n} d_{k} a_{k}+\Delta t \sum_{k=0}^{n} c_{k}+C_{0}, \quad \forall n \geq 1
$$

Then,

$$
a_{n}+\Delta t \sum_{k=0}^{n} b_{k} \leq\left(\Delta t \sum_{k=0}^{n} c_{k}+C_{0}\right) \exp \left(\Delta t \sum_{k=0}^{n} d_{k}\right) \quad \forall n \geq 1 .
$$

Following the proofs provided in $[1,12,14,21]$, we can obtain that problem (12) possesses a unique solution $(u, p, \theta)$ which satisfies the following regularity results under some nonlocal compatibility assumptions at $t=0$.

Theorem 2. Let $f \in L^{\infty}(0, T ; Y), f_{t} \in L^{2}(0, T ; Y), g \in$ $L^{\infty}(0, T, Z), g_{t} \in L^{2}(0, T, Z)$, and $u_{0} \in D(A) \cap V, \theta_{0} \in E(A)$. Then for all $T>0$ and $0<t \leq T$ the solution $(u, p, \theta)$ of problem (12) satisfies

$$
\begin{gathered}
\|A u(t)\|_{0}+\left\|\nabla u_{t}(t)\right\|_{0}+\left\|A u_{t}(t)\right\|_{0}+\|A \theta(t)\|_{0} \\
+\left\|\nabla \theta_{t}(t)\right\|_{0}+\left\|A \theta_{t}(t)\right\|_{0} \leq C
\end{gathered}
$$


We introduce the following inequalities:

$$
\begin{gathered}
\|v\|_{0} \leq C_{3}\|\nabla v\|_{0} \quad \forall v \in X \text { or } W ; \\
\|\nabla v\|_{0} \leq C_{4}\|A v\|_{0} \quad \forall v \in D(A) \text { or } H^{2}(\Omega) .
\end{gathered}
$$

We end this section by recalling some properties of the trilinear forms $b(\cdot, \cdot, \cdot)$ and $\bar{b}(\cdot, \cdot, \cdot)$, which can be found in $[1$, $11,13,14,22]$.

Lemma 3. The trilinear forms $b$ and $\bar{b}$ satisfy the following.

(1) In view of $H^{1}(\Omega) \hookrightarrow L^{4}(\Omega)$, one has

$$
\begin{array}{ll}
|b(u, v, w)| \leq N\|\nabla u\|_{0}\|\nabla v\|_{0}\|\nabla w\|_{0}, & \forall u, v, w \in X, \\
|\bar{b}(u, \theta, \psi)| \leq \bar{N}\|\nabla u\|_{0}\|\nabla \theta\|_{0}\|\nabla \psi\|_{0}, \quad \forall u \in X, \theta, \psi \in W,
\end{array}
$$

where

$$
\begin{aligned}
& N=\sup _{0 \neq u, v, w \in X} \frac{|b(u, v, w)|}{\|\nabla u\|_{0}\|\nabla v\|_{0}\|\nabla w\|_{0}}, \\
& \bar{N}=\sup _{0 \neq u \in X, 0 \neq \theta, \psi \in W} \frac{|\bar{b}(u, \theta, \psi)|}{\|\nabla u\|_{0}\|\nabla \theta\|_{0}\|\nabla \psi\|_{0}} .
\end{aligned}
$$

(2) Under the condition of $\operatorname{div} u=0$,

$$
\begin{array}{ll}
b(u, v, v)=0 & \forall u, v \in X \\
\bar{b}(u, \theta, \theta)=0 & \forall u \in X, \theta \in W .
\end{array}
$$

(3) The following estimates about trilinear terms $b(\cdot, \cdot, \cdot)$ and $\bar{b}(\cdot, \cdot, \cdot)$ hold:

$$
\begin{gathered}
|b(u, v, w)| \leq C_{5}\|A v\|_{0}\|u\|_{1}\|w\|_{0}, \\
\forall u \in V, v \in D(A), w \in X, \\
|b(u, v, w)| \leq C_{5}\|u\|_{0}^{1 / 2}\|A u\|_{0}^{1 / 2}\|v\|_{1}\|w\|_{0}, \\
\forall u \in D(A), v \in V, w \in X, \\
|\bar{b}(u, \theta, \psi)| \leq C_{6}\|A \theta\|_{0}\|u\|_{1}\|\psi\|_{0}, \\
\forall u \in V, \theta \in E(A), \psi \in W, \\
|\bar{b}(u, \theta, \psi)| \leq C_{6}\|u\|_{0}^{1 / 2}\|A u\|_{0}^{1 / 2}\|\theta\|_{1}\|\psi\|_{0}, \\
\forall u \in D(A), \theta, \psi \in W .
\end{gathered}
$$

\section{The Coupled and Decoupled Algorithms for Time-Dependent Natural Convection Problem}

In this section, let $\Delta t>0$ be the time step and $t_{n}=$ $n \Delta t ; u^{n}$ and $\theta^{n}$ denote the numerical solutions of $u$ and $\theta$ at $t_{n}$, respectively. We consider the backward Euler time discretization schemes for problem (1). Our schemes consist of two kinds of numerical schemes. One is the coupled scheme; the other is the decoupled scheme; these numerical algorithms are formulated as follows.
3.1. Coupled Algorithm for Time-Dependent Natural Convection Problem. The coupled time semidiscrete scheme for time-dependent natural convection problem (1) based on the backward Euler scheme can be written as

$$
\begin{gathered}
\frac{u^{n+1}-u^{n}}{\Delta t}-v \Delta u^{n+1}+\left(u^{n+1} \cdot \nabla\right) u^{n+1}+\nabla p^{n+1} \\
=-k v^{2} j \theta^{n+1}+f\left(t_{n+1}\right), \\
\nabla \cdot u^{n+1}=0, \\
\frac{\theta^{n+1}-\theta^{n}}{\Delta t}-\lambda v \Delta \theta^{n+1}+\left(u^{n+1} \cdot \nabla\right) \theta^{n+1}=g\left(t_{n+1}\right)
\end{gathered}
$$

with $0 \leq n<N=[T / \Delta t]$. The superscript $n$ denotes the time level $t_{n}$. The system (27) is a nonlinear problem; the weak form of (27) can be formulated as for all $(v, q, \psi) \in X \times M \times W$

$$
\begin{aligned}
& \left(\frac{u^{n+1}-u^{n}}{\Delta t}, v\right)+v\left(\nabla u^{n+1}, \nabla v\right)+b\left(u^{n+1}, u^{n+1}, v\right) \\
& +\left(v, \nabla p^{n+1}\right)=-k v^{2}\left(j \theta^{n+1}, v\right)+\left(f\left(t_{n+1}\right), v\right), \\
& \left(\nabla \cdot u^{n+1}, q\right)=0, \\
& \left(\frac{\theta^{n+1}-\theta^{n}}{\Delta t}, \psi\right)+\lambda v\left(\nabla \theta^{n+1}, \nabla \psi\right)+\bar{b}\left(u^{n+1}, \theta^{n+1}, \psi\right) \\
& =\left(g\left(t_{n+1}\right), \psi\right) .
\end{aligned}
$$

The existence and uniqueness of $u^{n+1}, p^{n+1}$, and $\theta^{n+1}$ have been established by Luo in [21]. From the expression of (28), we can see that when we solve problem (28) numerically, a large nonlinear algebra system should be solved, and the coefficient matrix is asymmetric. In general, it is expensive to solve such a nonlinear and coupled system. In order to improve the computational efficiency, we develop a decoupled and linearized scheme for problem (1).

3.2. Decoupled Algorithm for Time-Dependent Natural Convection Problem. The decoupled and linearized semidiscrete scheme for time-dependent natural convection problem (1) based on the backward Euler scheme can be presented as follows:

$$
\begin{gathered}
\frac{u^{d, n+1}-u^{d, n}}{\Delta t}-v \Delta u^{d, n+1}+\left(u^{d, n} \cdot \nabla\right) u^{d, n+1}+\nabla p^{d, n+1} \\
=-k v^{2} j \theta^{d, n}+f\left(t_{n+1}\right), \\
\nabla \cdot u^{d, n+1}=0, \\
\frac{\theta^{d, n+1}-\theta^{d, n}}{\Delta t}-\lambda \nu \Delta \theta^{d, n+1}+\left(u^{d, n} \cdot \nabla\right) \theta^{d, n+1}=g\left(t_{n+1}\right) .
\end{gathered}
$$


Scheme (29) is a mixed problem that contains a generalized Stokes problem and an elliptic problem. Namely, problem (29) can be split into two subproblems:

$$
\begin{gathered}
\frac{u^{d, n+1}-u^{d, n}}{\Delta t}-v \Delta u^{d, n+1}+\left(u^{d, n} \cdot \nabla\right) u^{d, n+1}+\nabla p^{d, n+1} \\
=-k v^{2} j \theta^{d, n}+f\left(t_{n+1}\right), \\
\nabla \cdot u^{d, n+1}=0, \\
\frac{\theta^{d, n+1}-\theta^{d, n}}{\Delta t}-\lambda v \Delta \theta^{d, n+1}+\left(u^{d, n} \cdot \nabla\right) \theta^{d, n+1}=g\left(t_{n+1}\right) .
\end{gathered}
$$

The weak form of problem (29) consists of finding $u^{d, n+1} \in$ $L^{2}(0, T ; X), p^{d, n+1} \in L^{2}(0, T ; M)$, and $\theta^{d, n+1} \in L^{2}(0, T ; W)$ such that for all $(v, q, \psi) \in X \times M \times W$,

$$
\begin{aligned}
& \left(\frac{u^{d, n+1}-u^{d, n}}{\Delta t}, v\right)+v\left(\nabla u^{d, n+1}, \nabla v\right)+b\left(u^{d, n}, u^{d, n+1}, v\right) \\
& +\left(v, \nabla p^{d, n+1}\right)=-k v^{2}\left(j \theta^{d, n}, v\right)+\left(f\left(t_{n+1}\right), v\right), \\
& \left(\nabla \cdot u^{d, n+1}, q\right)=0, \\
& \left(\frac{\theta^{d, n+1}-\theta^{d, n}}{\Delta t}, \psi\right)+\lambda v\left(\nabla \theta^{d, n+1}, \nabla \psi\right)+\bar{b}\left(u^{d, n}, \theta^{d, n+1}, \psi\right) \\
& =\left(g\left(t_{n+1}\right), \psi\right) .
\end{aligned}
$$

If $\left(\nabla \cdot u^{d, n+1}, q\right)$ satisfies the so-called inf-sup condition,

$$
\beta\|q\|_{0} \leq \inf _{q \in M} \sup _{0 \neq u \in X} \frac{\left(\nabla \cdot u^{d, n+1}, q\right)}{\left\|\nabla u^{d, n+1}\right\|_{0}} \quad(\beta>0 \text { is a constant })
$$

we can obtain the existence and uniqueness of $u^{d, n+1}, p^{d, n+1}$, and $\theta^{d, n+1}$ by $[13,14]$. From the expressions of $(30)$ we know that the variables $u^{d, n+1}, p^{d, n+1}$, and $\theta^{d, n+1}$ can be solved separately with two small linear algebra systems (30). Furthermore, the coefficient matrices of subproblems (30) are symmetric. It is much easier to solve these two subproblems than the original one.

\section{Stabilities of the Coupled and Decoupled Algorithms}

In this section, we consider the stabilities of both the coupled and decoupled numerical schemes under some assumptions presented in Section 2.
Lemma 4. Under assumptions (A3)-(A7), the following results of scheme (28) hold for all $J=0,1, \ldots,[T / \Delta t]-1$ :

$$
\begin{gathered}
\left\|u^{J+1}\right\|_{0}^{2}+\sum_{n=0}^{J}\left\|u^{n+1}-u^{n}\right\|_{0}^{2}+\Delta t v \sum_{n=0}^{J}\left\|\nabla u^{n+1}\right\|_{0}^{2} \leq \gamma_{0}^{2}, \\
\left\|\theta^{J+1}\right\|_{0}^{2}+\sum_{n=0}^{J}\left\|\theta^{n+1}-\theta^{n}\right\|_{0}^{2}+\lambda \nu \Delta t \sum_{n=0}^{J}\left\|\nabla \theta^{n+1}\right\|_{0}^{2} \leq \gamma_{1}^{2},
\end{gathered}
$$

where $\gamma_{0}^{2}=\left(2 C_{3}^{4} k \nu^{2} / \lambda\right)\left(\left(C_{3}^{2} / \lambda \nu\right) T g_{\infty}^{2}+\left\|\theta^{0}\right\|_{0}^{2}\right)+2 C_{3}^{2} f_{\infty}^{2} T / \nu+$ $\left\|u^{0}\right\|_{0}^{2}, \gamma_{1}^{2}=C_{3}^{2} T g_{\infty}^{2} / \lambda \nu+\left\|\theta^{0}\right\|_{0}^{2}$.

Proof. Taking $v=2 \Delta t u^{n+1}, q=2 \Delta t p^{n+1}$, and $\psi=2 \Delta t \theta^{n+1}$ in (28) we derive that

$$
\begin{gathered}
\left(u^{n+1}-u^{n}, 2 u^{n+1}\right)+2 \Delta t \nu\left\|\nabla u^{n+1}\right\|_{0}^{2} \\
=-2 k \nu^{2} \Delta t\left(j \theta^{n+1}, u^{n+1}\right)+2 \Delta t\left(f\left(t_{n+1}\right), u^{n+1}\right), \\
\left(\theta^{n+1}-\theta^{n}, 2 \theta^{n+1}\right)+2 \Delta t \lambda \nu\left\|\nabla \theta^{n+1}\right\|_{0}^{2}=2 \Delta t\left(g\left(t_{n+1}\right), \theta^{n+1}\right) .
\end{gathered}
$$

By using the identities

$$
\begin{gathered}
(a-b, 2 a)=|a|^{2}-|b|^{2}+|a-b|^{2}, \\
2(a, b)=|a|^{2}+|b|^{2}-|a-b|^{2},
\end{gathered}
$$

problem (34) can be transformed into

$$
\begin{aligned}
& \left\|u^{n+1}\right\|_{0}^{2}-\left\|u^{n}\right\|_{0}^{2}+\left\|u^{n+1}-u^{n}\right\|_{0}^{2}+2 \Delta t \nu\left\|\nabla u^{n+1}\right\|_{0}^{2} \\
& =-2 k \nu^{2} \Delta t\left(j \theta^{n+1}, u^{n+1}\right)+2 \Delta t\left(f\left(t_{n+1}\right), u^{n+1}\right), \\
& \left\|\theta^{n+1}\right\|_{0}^{2}-\left\|\theta^{n}\right\|_{0}^{2}+\left\|\theta^{n+1}-\theta^{n}\right\|_{0}^{2}+2 \Delta t \lambda \nu\left\|\nabla \theta^{n+1}\right\|_{0}^{2} \\
& =2 \Delta t\left(g\left(t_{n+1}\right), \theta^{n+1}\right) .
\end{aligned}
$$

For the right-hand side terms of (36) we have

$$
\begin{aligned}
\left|2 k v^{2} \Delta t\left(j \theta^{n+1}, u^{n+1}\right)\right| & \leq 2 k v^{2} \Delta t\left\|\theta^{n+1}\right\|_{0}\left\|u^{n+1}\right\|_{0} \\
& \leq \frac{v \Delta t}{2}\left\|\nabla u^{n+1}\right\|_{0}^{2}+2 C_{3}^{2} k^{2} v^{3} \Delta t\left\|\theta^{n+1}\right\|_{0}^{2}, \\
\left|2 \Delta t\left(f\left(t_{n+1}\right), u^{n+1}\right)\right| & \leq 2 \Delta t\left\|f\left(t_{n+1}\right)\right\|_{0}\left\|u^{n+1}\right\|_{0} \\
& \leq \frac{v \Delta t}{2}\left\|\nabla u^{n+1}\right\|_{0}^{2}+\frac{2 C_{3}^{2}}{v} \Delta t\left\|f\left(t_{n+1}\right)\right\|_{0}^{2}, \\
2\left|\Delta t\left(g\left(t_{n+1}\right), \theta^{n+1}\right)\right| & \leq 2 C_{3} \Delta t\left\|g\left(t_{n+1}\right)\right\|_{0}\left\|\nabla \theta^{n+1}\right\|_{0} \\
& \leq \lambda v \Delta t\left\|\nabla \theta^{n+1}\right\|_{0}^{2}+\frac{C_{3}^{2} \Delta t}{\lambda \nu}\left\|g\left(t_{n+1}\right)\right\|_{0}^{2} .
\end{aligned}
$$


Combining above estimates with (36) and summing them for $n$ from 0 to $J$ yield

$$
\begin{aligned}
& \left\|u^{J+1}\right\|_{0}^{2}+\sum_{n=0}^{J}\left\|u^{n+1}-u^{n}\right\|_{0}^{2}+\Delta t \nu \sum_{n=0}^{J}\left\|\nabla u^{n+1}\right\|_{0}^{2} \\
& \leq 2 C_{3}^{2} k^{2} \nu^{3} \Delta t \sum_{n=0}^{J}\left\|\theta^{n+1}\right\|_{0}^{2}+\frac{2 C_{3}^{2}}{\nu} \Delta t \sum_{n=0}^{J}\left\|f\left(t_{n+1}\right)\right\|_{0}^{2}+\left\|u^{0}\right\|_{0}^{2},
\end{aligned}
$$

$$
\begin{aligned}
& \left\|\theta^{J+1}\right\|_{0}^{2}+\sum_{n=0}^{J}\left\|\theta^{n+1}-\theta^{n}\right\|_{0}^{2}+\lambda v \Delta t \sum_{n=0}^{J}\left\|\nabla \theta^{n+1}\right\|_{0}^{2} \\
& \leq \frac{C_{3}^{2}}{\lambda v} \sum_{n=0}^{J} \Delta t\left\|g\left(t_{n+1}\right)\right\|_{0}^{2}+\left\|\theta^{0}\right\|_{0}^{2} \leq \frac{C_{3}^{2} T g_{\infty}^{2}}{\lambda \nu}+\left\|\theta^{0}\right\|_{0}^{2} .
\end{aligned}
$$

We complete the proof by substituting (39) into (38) and using (22).

Lemma 5. Under assumptions (A3)-(A7), the following results of scheme (28) hold for all $J=0,1, \ldots,[T / \Delta t]-1$ :

$$
\begin{aligned}
& \frac{1}{4 v} \sum_{n=0}^{J}\left\|d_{t} u^{n+1}\right\|_{0}^{2} \Delta t+\left\|\nabla u^{J+1}\right\|_{0}^{2} \\
& \quad+\sum_{n=0}^{J}\left(\left\|\nabla\left(u^{n+1}-u^{n}\right)\right\|_{0}^{2}+\frac{\Delta t v}{4}\left\|A u^{n+1}\right\|_{0}^{2}\right) \leq \gamma_{2}^{2}, \\
& \frac{1}{4 \lambda v} \sum_{n=0}^{J}\left\|d_{t} \theta^{n+1}\right\|_{0}^{2} \Delta t+\left\|\nabla \theta^{J+1}\right\|_{0}^{2} \\
& \quad+\sum_{n=0}^{J}\left(\left\|\nabla\left(\theta^{n+1}-\theta^{n}\right)\right\|_{0}^{2}+\frac{\lambda \nu \Delta t}{4}\left\|A \theta^{n+1}\right\|_{0}^{2}\right) \leq \gamma_{3}^{2},
\end{aligned}
$$

where $\gamma_{2}^{2}=\exp \left(v^{-1}(8 / v)^{3} C_{5}^{4} \gamma_{0}^{4}\right)\left(2\left\|\nabla u^{0}\right\|_{0}^{2}+2\left\|\nabla \theta^{0}\right\|_{0}^{2}+\right.$ $\left.(10 / \nu) T f_{\infty}^{2}+10 C_{3}^{4} T g_{\infty}^{2} k^{2} \nu^{3} / \lambda^{2} \nu^{3}\right)$ and $\gamma_{3}^{2}=\exp \left((8 / \lambda \nu)^{3}\right.$ $\left.C_{3}^{2} C_{6}^{4} \gamma_{0}^{4}\right)\left(2\left\|\nabla \theta^{0}\right\|_{0}^{2}+(10 / \lambda \nu) T g_{\infty}^{2}\right)$.

Proof. In order to simplify the notations, we set $d_{t} \varphi^{n+1}=$ $\left(\varphi^{n+1}-\varphi^{n}\right) / \Delta t, \varphi$ taking $u$ or $\theta$. Choose $v=\left(v^{-1} d_{t} u^{n+1}+\right.$ $\left.A u^{n+1}\right) \Delta t \in V$ and $\psi=\left((\lambda \nu)^{-1} d_{t} \theta^{n+1}+A \theta^{n+1}\right) \Delta t$ in (28) to obtain

$$
\begin{aligned}
v^{-1} \Delta t & d_{t} u^{n+1}\left\|_{0}^{2}+\right\| \nabla u^{n+1}\left\|_{0}^{2}-\right\| \nabla u^{n}\left\|_{0}^{2}+v \Delta t\right\| A u^{n+1} \|_{0}^{2} \\
& +b\left(u^{n+1}, u^{n+1}, v^{-1} d_{t} u^{n+1}+A u^{n+1}\right) \Delta t \\
& +\left\|\nabla\left(u^{n+1}-u^{n}\right)\right\|_{0}^{2} \\
= & \left(f\left(t_{n+1}\right), v^{-1} d_{t} u^{n+1}+A u^{n+1}\right) \Delta t \\
& -k \nu^{2} \Delta t\left(j \theta^{n+1}, v^{-1} d_{t} u^{n+1}+A u^{n+1}\right), \\
(\lambda \nu)^{-1} \Delta t & \left\|d_{t} \theta^{n+1}\right\|_{0}^{2}+\left\|\nabla \theta^{n+1}\right\|_{0}^{2}-\left\|\nabla \theta^{n}\right\|_{0}^{2}
\end{aligned}
$$

$$
\begin{aligned}
& +\left\|\nabla\left(\theta^{n+1}-\theta^{n}\right)\right\|_{0}^{2}+\lambda \nu \Delta t\left\|A \theta^{n+1}\right\|_{0}^{2} \\
& +\bar{b}\left(u^{n+1}, \theta^{n+1},(\lambda \nu)^{-1} d_{t} \theta^{n+1}+A \theta^{n+1}\right) \Delta t \\
= & \left(g\left(t_{n+1}\right),(\lambda v)^{-1} d_{t} \theta^{n+1}+A \theta^{n+1}\right) \Delta t .
\end{aligned}
$$

Using Lemma 3 and Cauchy inequality we arrive at

$$
\begin{aligned}
& \left|b\left(u^{n+1}, u^{n+1}, v^{-1} d_{t} u^{n+1}+A u^{n+1}\right)\right| \\
& \leq C_{5}\left\|A^{1 / 2} u^{n+1}\right\|_{0}\left\|u^{n+1}\right\|_{0}^{1 / 2}\left\|A u^{n+1}\right\|_{0}^{1 / 2} \\
& \times\left(v^{-1}\left\|d_{t} u^{n+1}\right\|_{0}+\left\|A u^{n+1}\right\|_{0}\right) \\
& \leq \frac{1}{4 \nu}\left\|d_{t} u^{n+1}\right\|_{0}^{2}+\frac{v}{4}\left\|A u^{n+1}\right\|_{0}^{2} \\
& +\frac{8}{v} C_{5}^{2}\left\|A^{1 / 2} u^{n+1}\right\|_{0}^{2}\left\|u^{n+1}\right\|_{0}\left\|A u^{n+1}\right\|_{0} \\
& \leq \frac{1}{4 v}\left\|d_{t} u^{n+1}\right\|_{0}^{2}+\frac{3 v}{4}\left\|A u^{n+1}\right\|_{0}^{2} \\
& +\frac{1}{2}\left(\frac{8}{v}\right)^{3} C_{5}^{4}\left\|A^{1 / 2} u^{n+1}\right\|_{0}^{4}\left\|u^{n+1}\right\|_{0}^{2}, \\
& \left|\bar{b}\left(u^{n+1}, \theta^{n+1},(\lambda \nu)^{-1} d_{t} \theta^{n+1}+A \theta^{n+1}\right)\right| \\
& \leq C_{6}\left\|A^{1 / 2} u^{n+1}\right\|_{0}\left\|\theta^{n+1}\right\|_{0}^{1 / 2}\left\|A \theta^{n+1}\right\|_{0}^{1 / 2} \\
& \times\left((\lambda \nu)^{-1}\left\|d_{t} \theta^{n+1}\right\|_{0}+\left\|A \theta^{n+1}\right\|_{0}\right) \\
& \leq \frac{1}{4 \lambda \nu}\left\|d_{t} \theta^{n+1}\right\|_{0}^{2}+\frac{\lambda \nu}{4}\left\|A \theta^{n+1}\right\|_{0}^{2} \\
& +\frac{8}{\lambda \nu} C_{6}^{2}\left\|A^{1 / 2} u^{n+1}\right\|_{0}^{2}\left\|\theta^{n+1}\right\|_{0}\left\|A \theta^{n+1}\right\|_{0} \\
& \leq \frac{1}{4 \lambda \nu}\left\|d_{t} \theta^{n+1}\right\|_{0}^{2}+\frac{3 \lambda \nu}{4}\left\|A \theta^{n+1}\right\|_{0}^{2} \\
& +\frac{1}{2}\left(\frac{8}{\lambda \nu}\right)^{3} C_{6}^{4}\left\|A^{1 / 2} u^{n+1}\right\|_{0}^{4}\left\|\theta^{n+1}\right\|_{0}^{2}, \\
& \left|\left(f\left(t_{n+1}\right), v^{-1} d_{t} u^{n+1}+A u^{n+1}\right)\right| \\
& \leq \frac{1}{4 v}\left\|d_{t} u^{n+1}\right\|_{0}^{2}+\frac{v}{16}\left\|A u^{n+1}\right\|_{0}^{2}+\frac{5}{v}\left\|f\left(t_{n+1}\right)\right\|_{0}^{2}, \\
& \left|k \nu^{2}\left(j \theta^{n+1}, \nu^{-1} d_{t} u^{n+1}+A u^{n+1}\right)\right| \\
& \leq \frac{1}{4 v}\left\|d_{t} u^{n+1}\right\|_{0}^{2}+\frac{v}{16}\left\|A u^{n+1}\right\|_{0}^{2}+5 k^{2} \nu^{3}\left\|\theta^{n+1}\right\|_{0}^{2}, \\
& \left|\left(g\left(t_{n+1}\right),(\lambda v)^{-1} d_{t} \theta^{n+1}+A \theta^{n+1}\right)\right| \\
& \leq \frac{1}{4 \lambda \nu}\left\|d_{t} \theta^{n+1}\right\|_{0}^{2}+\frac{\lambda \nu}{16}\left\|A \theta^{n+1}\right\|_{0}^{2}+\frac{5}{\lambda \nu}\left\|g\left(t_{n+1}\right)\right\|_{0}^{2} .
\end{aligned}
$$


Combining above inequalities with (41) one finds

$$
\begin{aligned}
(2 v)^{-1} \Delta & \left\|d_{t} u^{n+1}\right\|_{0}^{2}+2\left\|\nabla u^{n+1}\right\|_{0}^{2}-2\left\|\nabla u^{n}\right\|_{0}^{2} \\
& +2\left\|\nabla\left(u^{n+1}-u^{n}\right)\right\|_{0}^{2}+\frac{\nu}{4} \Delta t\left\|A u^{n+1}\right\|_{0}^{2} \\
\leq & \left(\frac{8}{v}\right)^{3} C_{5}^{4}\left\|\nabla u^{n+1}\right\|_{0}^{2}\left\|u^{n+1}\right\|_{0}^{2}\left\|\nabla u^{n+1}\right\|_{0}^{2} \Delta t \\
& +\frac{10}{\nu}\left\|f\left(t_{n+1}\right)\right\|_{0}^{2} \Delta t+10 k^{2} v^{3}\left\|\theta^{n+1}\right\|_{0}^{2} \Delta t, \\
(2 \lambda \nu)^{-1} & \Delta t\left\|d_{t} \theta^{n+1}\right\|_{0}^{2}+2\left\|\nabla \theta^{n+1}\right\|_{0}^{2}-2\left\|\nabla \theta^{n}\right\|_{0}^{2} \\
& +2\left\|\nabla\left(\theta^{n+1}-\theta^{n}\right)\right\|_{0}^{2}+\frac{3 \lambda \nu}{4} \Delta t\left\|A \theta^{n+1}\right\|_{0}^{2} \\
\leq & \left(\frac{8}{\lambda \nu}\right)^{3} C_{6}^{4}\left\|^{n+1}\right\|_{0}^{2}\left\|\theta^{n+1}\right\|_{0}^{2}\left\|\nabla u^{n+1}\right\|_{0}^{2} \Delta t \\
& +\frac{10}{\lambda \nu}\left\|g\left(t_{n+1}\right)\right\|_{0}^{2} \Delta t .
\end{aligned}
$$

Summing (43) for $n$ from 0 to $J$, using Lemmas 1 and 4, we have

$$
\begin{gathered}
\Delta t \sum_{n=0}^{J}\left((2 v)^{-1}\left\|d_{t} u^{n+1}\right\|_{0}^{2}+\frac{\nu}{4}\left\|A u^{n+1}\right\|_{0}^{2}\right)+2\left\|\nabla u^{J+1}\right\|_{0}^{2} \\
+2 \sum_{n=0}^{J}\left\|\nabla\left(u^{n+1}-u^{n}\right)\right\|_{0}^{2} \\
\leq \exp \left(v^{-1}\left(\frac{8}{\nu}\right)^{3} C_{5}^{4} \gamma_{0}^{4}\right)\left(2\left\|\nabla u^{0}\right\|_{0}^{2}+\frac{10}{\nu} T f_{\infty}^{2}\right. \\
\left.+10 k^{2} \nu^{3} \sum_{n=0}^{J}\left\|\theta^{n+1}\right\|_{0}^{2} \Delta t\right) \\
\Delta t \sum_{n=0}^{J}\left((2 \lambda \nu)^{-1}\left\|d_{t} \theta^{n+1}\right\|_{0}^{2}+\frac{\lambda \nu}{4}\left\|A \theta^{n+1}\right\|_{0}^{2}\right) \\
+2\left\|\nabla \theta^{J+1}\right\|_{0}^{2}+2 \sum_{n=0}^{J}\left\|\nabla\left(\theta^{n+1}-\theta^{n}\right)\right\|_{0}^{2} \\
\leq \exp \left(\left(\frac{8}{\lambda \nu}\right)^{3} C_{3}^{2} C_{6}^{4} \gamma_{0}^{4}\right)\left(2\left\|\nabla \theta^{0}\right\|_{0}^{2}+\frac{10}{\lambda \nu} T g_{\infty}^{2}\right)
\end{gathered}
$$

which together with Lemma 4 implies the desired results.

Furthermore, following the proofs provided in $[23,24]$, we obtain the following stability results for the numerical solutions $u^{d, n}$ and $\theta^{d, n}$ of the decoupled numerical scheme (31).
Lemma 6. Under assumptions (A3)-(A7), the following results of problem (31) hold:

$$
\begin{aligned}
& \left\|u^{d, J+1}\right\|_{0}^{2}+\sum_{n=0}^{J}\left\|u^{d, n+1}-u^{d, n}\right\|_{0}^{2}+\Delta t v \sum_{n=0}^{J}\left\|\nabla u^{d, n+1}\right\|_{0}^{2} \\
& \leq \gamma_{0}^{2} \text {, } \\
& \left\|\theta^{d, J+1}\right\|_{0}^{2}+\sum_{n=0}^{J}\left\|\theta^{d, n+1}-\theta^{d, n}\right\|_{0}^{2}+\lambda \nu \Delta t \sum_{n=0}^{J}\left\|\nabla \theta^{d, n+1}\right\|_{0}^{2} \\
& \leq \gamma_{1}^{2} \\
& \left\|\nabla u^{d, J+1}\right\|_{0}^{2}+\Delta t \nu \sum_{n=0}^{J}\left(\left\|\nabla\left(u^{d, n+1}-u^{d, n}\right)\right\|_{0}^{2}+\frac{1}{4 \nu^{2}}\left\|d_{t} u^{d, n+1}\right\|_{0}^{2}\right. \\
& \left.+\frac{1}{4}\left\|A u^{d, n+1}\right\|_{0}^{2}\right) \leq \gamma_{4}^{2} \\
& \left\|\nabla \theta^{d, J+1}\right\|_{0}^{2} \\
& +\lambda \nu \Delta t \sum_{n=0}^{J}\left(\left\|\nabla\left(\theta^{d, n+1}-\theta^{d, n}\right)\right\|_{0}^{2}\right. \\
& \left.+\frac{1}{4(\lambda \nu)^{2}}\left\|d_{t} \theta^{d, n+1}\right\|_{0}^{2}+\frac{1}{4}\left\|A \theta^{d, n+1}\right\|_{0}^{2}\right) \leq \gamma_{5}^{2}
\end{aligned}
$$

for all $J=0,1, \ldots,[T / \Delta t]-1$, where $\gamma_{4}^{2}=\exp \left(\nu^{-1} 4 C_{5}^{2} \gamma_{0}^{2}\right)$ $\left(\left\|\nabla u^{0}\right\|_{0}^{2}+(3 / \nu) T f_{\infty}^{2}+3 k^{2} \nu^{3} \gamma_{1}^{2} T\right)$ and $\gamma_{5}^{2}=\exp \left((\lambda \nu)^{-3}\right.$ $\left.\gamma_{1}^{2} \gamma_{4}^{2}\right)\left(\left\|\nabla \theta^{0}\right\|_{0}^{2}+(2 / \lambda \nu) T g_{\infty}^{2}\right)$.

\section{Error Estimates of the Coupled Numerical Scheme}

This section is devoted to present the optimal error estimates of velocity, pressure, and temperature in the coupled numerical scheme (28) introduced in Section 3. In order to simplify the descriptions, we denote

$$
\begin{gathered}
E_{u}^{n}=u\left(t_{n}\right)-u^{n}, \quad E_{p}^{n}=p\left(t_{n}\right)-p^{n}, \\
E_{\theta}^{n}=\theta\left(t_{n}\right)-\theta^{n} .
\end{gathered}
$$

(a) Error estimates for velocity and temperature in scheme (28) are as follows.

Let us define the truncation errors $R_{u}^{n}$ and $R_{\theta}^{n}$ by

$$
\begin{aligned}
& \frac{u\left(t_{n+1}\right)-u\left(t_{n}\right)}{\Delta t}-v \Delta u\left(t_{n+1}\right)+\left(u\left(t_{n+1}\right) \cdot \nabla\right) u\left(t_{n+1}\right) \\
& +\nabla p\left(t_{n+1}\right)=-k \nu^{2} j \theta\left(t_{n+1}\right)+f\left(t_{n+1}\right)+R_{u}^{n}, \\
& \nabla \cdot u\left(t_{n+1}\right)=0 \\
& \frac{\theta\left(t_{n+1}\right)-\theta\left(t_{n}\right)}{\Delta t}-\lambda \nu \Delta \theta\left(t_{n+1}\right)+\left(u\left(t_{n+1}\right) \cdot \nabla\right) \theta\left(t_{n+1}\right) \\
& =g\left(t_{n+1}\right)+R_{\theta}^{n}
\end{aligned}
$$


where

$$
\begin{aligned}
& R_{u}^{n}=-\frac{1}{\Delta t} \int_{t_{n}}^{t_{n+1}}\left(t-t_{n}\right) u_{t t}(t) d t, \\
& R_{\theta}^{n}=-\frac{1}{\Delta t} \int_{t_{n}}^{t_{n+1}}\left(t-t_{n}\right) \theta_{t t}(t) d t .
\end{aligned}
$$

Firstly, we present the estimates of $E_{u}^{n+1}$ and $E_{\theta}^{n+1}$ which show that both $u^{n+1}$ and $\theta^{n+1}$ are order $1 / 2$ approximations to $u$ and $\theta$ in $L^{\infty}(Y)$ and in $L^{\infty}(Z)$, respectively.

Lemma 7. Assume that assumptions (A3)-(A6) hold. Then for all $J=0,1, \ldots,[T / \Delta t]-1$ one has

$$
\begin{aligned}
& \left\|E_{u}^{J+1}\right\|_{0}^{2}+\sum_{n=0}^{J}\left\|E_{u}^{n+1}-E_{u}^{n}\right\|_{0}^{2}+v \Delta t \sum_{n=0}^{J}\left\|\nabla E_{u}^{n+1}\right\|_{0}^{2} \leq C \Delta t, \\
& \left\|E_{\theta}^{J+1}\right\|_{0}^{2}+\sum_{n=0}^{J}\left\|E_{\theta}^{n+1}-E_{\theta}^{n}\right\|_{0}^{2}+\lambda v \Delta t \sum_{n=0}^{J}\left\|\nabla E_{\theta}^{n+1}\right\|_{0}^{2} \leq C \Delta t .
\end{aligned}
$$

Proof. By subtracting (27) from (47) we have

$$
\begin{gathered}
\frac{E_{u}^{n+1}-E_{u}^{n}}{\Delta t}-v \Delta E_{u}^{n+1}+\left(u\left(t_{n+1}\right) \cdot \nabla\right) u\left(t_{n+1}\right) \\
-\left(u^{n+1} \cdot \nabla\right) u^{n+1}+\nabla E_{p}^{n+1}=-k v^{2} j E_{\theta}^{n+1}+R_{u}^{n}, \\
\nabla \cdot E_{u}^{n+1}=0, \\
\frac{E_{\theta}^{n+1}-E_{\theta}^{n}}{\Delta t}-\lambda v \Delta E_{\theta}^{n+1}+\left(u\left(t_{n+1}\right) \cdot \nabla\right) \theta\left(t_{n+1}\right) \\
-\left(u^{n+1} \cdot \nabla\right) \theta^{n+1}=R_{\theta}^{n} .
\end{gathered}
$$

Taking the inner product of (50) with $2 \Delta t E_{u}^{n+1}, 2 \Delta t E_{p}^{n+1}$, and $2 \Delta t E_{\theta}^{n+1}$, we can transform problem (50) into

$$
\begin{aligned}
&\left\|E_{u}^{n+1}\right\|_{0}^{2}-\left\|E_{u}^{n}\right\|_{0}^{2}+\left\|E_{u}^{n+1}-E_{u}^{n}\right\|_{0}^{2} \\
&+2 \Delta t b\left(E_{u}^{n+1}, u\left(t_{n+1}\right), E_{u}^{n+1}\right)+2 v \Delta t\left\|\nabla E_{u}^{n+1}\right\|_{0}^{2} \\
&=-2 k v^{2} \Delta t\left(j E_{\theta}^{n+1}, E_{u}^{n+1}\right)+2 \Delta t\left(R_{u}^{n}, E_{u}^{n+1}\right), \\
&\left\|E_{\theta}^{n+1}\right\|_{0}^{2}-\left\|E_{\theta}^{n}\right\|_{0}^{2}+\left\|E_{\theta}^{n+1}-E_{\theta}^{n}\right\|_{0}^{2}+2 \lambda v \Delta t\left\|\nabla E_{\theta}^{n+1}\right\|_{0}^{2} \\
&+2 \Delta t b\left(E_{u}^{n+1}, \theta\left(t_{n+1}\right), E_{\theta}^{n+1}\right)=2 \Delta t\left(R_{\theta}^{n}, E_{\theta}^{n+1}\right) .
\end{aligned}
$$

Now, the right-hand side terms of (51) can be treated as follows:

$$
\begin{aligned}
& \left|2 \Delta t\left(R_{u}^{n}, E_{u}^{n+1}\right)\right| \\
& \quad \leq \frac{C}{\Delta t}\left\|\int_{t_{n}}^{t_{n+1}}\left(t-t_{n}\right) u_{t t} d t\right\|_{0}^{2}+\frac{\nu \Delta t}{2}\left\|\nabla E_{u}^{n+1}\right\|_{0}^{2} \\
& \quad \leq C \Delta t \int_{t_{n}}^{t_{n+1}} t\left\|u_{t t}\right\|_{0}^{2} d t+\frac{\nu \Delta t}{2}\left\|\nabla E_{u}^{n+1}\right\|_{0}^{2},
\end{aligned}
$$

$$
\begin{aligned}
& \left|2 \Delta t\left(R_{\theta}^{n}, E_{\theta}^{n+1}\right)\right| \\
& \quad \leq \frac{C}{\Delta t}\left\|\int_{t_{n}}^{t_{n+1}}\left(t-t_{n}\right) \theta_{t t} d t\right\|_{0}^{2}+\frac{v \Delta t}{2}\left\|\nabla E_{\theta}^{n+1}\right\|_{0}^{2} \\
& \quad \leq C \Delta t \int_{t_{n}}^{t_{n+1}} t\left\|\theta_{t t}\right\|_{0}^{2} d t+\frac{v \Delta t}{2}\left\|\nabla E_{\theta}^{n+1}\right\|_{0}^{2}, \\
& \left|-2 k v^{2} \Delta t\left(j E_{\theta}^{n+1}, E_{u}^{n+1}\right)\right| \\
& \quad \leq 2 k v^{2} \Delta t\left\|E_{\theta}^{n+1}\right\|_{0}\left\|E_{u}^{n+1}\right\|_{0} \\
& \leq k^{2} v^{4} \Delta t\left\|E_{\theta}^{n+1}\right\|_{0}^{2}+\Delta t\left\|E_{u}^{n+1}\right\|_{0}^{2} .
\end{aligned}
$$

For the nonlinear term, thanks to Lemma 3, we have

$$
\begin{aligned}
\mid 2 \Delta t & \left(E_{u}^{n+1}, u\left(t_{n+1}\right), E_{u}^{n+1}\right) \mid \\
& \leq 2 C_{5} \Delta t\left\|E_{u}^{n+1}\right\|_{0}\left\|A u\left(t_{n+1}\right)\right\|_{0}\left\|\nabla E_{u}^{n+1}\right\|_{0} \\
& \leq \frac{2 C_{5}^{2}}{\nu} \Delta t\left\|E_{u}^{n+1}\right\|_{0}^{2}\left\|A u\left(t_{n+1}\right)\right\|_{0}^{2}+\frac{v \Delta t}{2}\left\|\nabla E_{u}^{n+1}\right\|_{0}^{2}, \\
\mid 2 \Delta t \bar{b} & \left(E_{u}^{n+1}, \theta\left(t_{n+1}\right), E_{\theta}^{n+1}\right) \mid \\
& \leq 2 C_{6} \Delta t\left\|E_{u}^{n+1}\right\|_{0}\left\|A \theta\left(t_{n+1}\right)\right\|_{0}\left\|\nabla E_{\theta}^{n+1}\right\|_{0} \\
& \leq \frac{2 C_{6}^{2}}{\lambda \nu} \Delta t\left\|E_{u}^{n+1}\right\|_{0}^{2}\left\|A \theta\left(t_{n+1}\right)\right\|_{0}^{2}+\frac{\lambda \nu \Delta t}{2}\left\|\nabla E_{\theta}^{n+1}\right\|_{0}^{2} .
\end{aligned}
$$

From above inequalities we arrive at

$$
\begin{gathered}
\left\|E_{u}^{n+1}\right\|_{0}^{2}-\left\|E_{u}^{n}\right\|_{0}^{2}+\left\|E_{u}^{n+1}-E_{u}^{n}\right\|_{0}^{2}+\nu \Delta t\left\|\nabla E_{u}^{n+1}\right\|_{0}^{2} \\
\leq\left(1+\frac{2 C_{5}^{2}\left\|A u\left(t_{n+1}\right)\right\|_{0}^{2}}{\nu}\right) \Delta t\left\|E_{u}^{n+1}\right\|_{0}^{2} \\
+C \Delta t \int_{t_{n}}^{t_{n+1}} t\left\|u_{t t}\right\|_{0}^{2} d t+k^{2} \nu^{4} \Delta t\left\|E_{\theta}^{n+1}\right\|_{0}^{2}, \\
\left\|E_{\theta}^{n+1}\right\|_{0}^{2}-\left\|E_{\theta}^{n}\right\|_{0}^{2}+\left\|E_{\theta}^{n+1}-E_{\theta}^{n}\right\|_{0}^{2}+\lambda v \Delta t\left\|\nabla E_{\theta}^{n+1}\right\|_{0}^{2} \\
\leq \frac{2 C_{6}^{2}}{\lambda \nu} \Delta t\left\|A \theta\left(t_{n+1}\right)\right\|_{0}^{2}\left\|E_{u}^{n+1}\right\|_{0}^{2}+C \Delta t \int_{t_{n}}^{t_{n+1}} t\left\|\theta_{t t}\right\|_{0}^{2} d t .
\end{gathered}
$$

Summing (55) from $n=0$ to $J$ one finds

$$
\begin{gathered}
\left\|E_{\theta}^{J+1}\right\|_{0}^{2}+\sum_{n=0}^{J}\left\|E_{\theta}^{n+1}-E_{\theta}^{n}\right\|_{0}^{2}+\lambda \nu \Delta t \sum_{n=0}^{J}\left\|\nabla E_{\theta}^{n+1}\right\|_{0}^{2} \\
\leq \frac{2 C_{6}^{2}}{\lambda \nu} \Delta t \sum_{n=0}^{J}\left\|A \theta\left(t_{n+1}\right)\right\|_{0}^{2}\left\|E_{u}^{n+1}\right\|_{0}^{2} \\
+C \Delta t \int_{0}^{T} t\left\|\theta_{t t}\right\|_{0}^{2} d t,
\end{gathered}
$$




$$
\begin{aligned}
\left\|E_{u}^{J+1}\right\|_{0}^{2} & +\sum_{n=0}^{J}\left\|E_{u}^{n+1}-E_{u}^{n}\right\|_{0}^{2}+v \Delta t \sum_{n=0}^{J}\left\|\nabla E_{u}^{n+1}\right\|_{0}^{2} \\
\leq & \Delta t \sum_{n=0}^{J}\left(1+\frac{2 C_{5}^{2}}{v}\left\|A u\left(t_{n+1}\right)\right\|_{0}^{2}\right)\left\|E_{u}^{n+1}\right\|_{0}^{2} \\
& +k^{2} v^{4} \Delta t \sum_{n=0}^{J}\left\|E_{\theta}^{n+1}\right\|_{0}^{2}+c \Delta t \int_{0}^{T} t\left\|u_{t t}\right\|_{0}^{2} d t .
\end{aligned}
$$

Combining Lemma 1 and Theorem 2 with (22) yields the desired results.

Remark 8. In particular, Lemma 7 shows that the coupled finite element method provides uniformly stable velocity and temperature in $X$ and $W$, respectively. Using the fact that $\left\|\nabla E_{u}^{n+1}\right\|_{0} \leq C,\left\|\nabla E_{\theta}^{n+1}\right\|_{0} \leq C$ and $u \in L^{\infty}(0, T ; X), \theta \in$ $L^{\infty}(0, T ; W)$, we know that there exists a positive constant $C$ independent of the time step $\Delta t$ such that for all $0 \leq n \leq$ $[T / \Delta t]-1$,

$$
\left\|\nabla u^{n+1}\right\|_{0} \leq C, \quad\left\|\nabla \theta^{n+1}\right\|_{0} \leq C .
$$

Lemma 9. For all $J=0,1, \ldots,[T / \Delta t]-1$, under assumptions (A3)-(A6), one has

$$
\begin{gathered}
\left\|E_{u}^{J+1}\right\|_{V^{\prime}}^{2}+\sum_{n=0}^{m}\left\|E_{u}^{n+1}-E_{u}^{n}\right\|_{V^{\prime}}^{2}+\frac{\nu \Delta t}{2} \sum_{n=0}^{J}\left\|E_{u}^{n+1}\right\|_{0}^{2} \leq C \Delta t^{2}, \\
\left\|E_{\theta}^{J+1}\right\|_{W^{\prime}}^{2}+\sum_{n=0}^{m}\left\|E_{\theta}^{n+1}-E_{\theta}^{n}\right\|_{W^{\prime}}^{2}+\frac{\lambda \nu \Delta t}{2} \sum_{n=0}^{J}\left\|E_{\theta}^{n+1}\right\|_{0}^{2} \leq C \Delta t^{2} .
\end{gathered}
$$

Proof. Taking the inner product of (50) with $2 \Delta t A^{-1} E_{u}^{n+1}$ and $2 \Delta t A^{-1} E_{\theta}^{n+1}$, using the fact that $\nabla \cdot E_{u}^{n+1}=0$ and the selfadjointness of $A^{-1}$, we obtain

$$
\begin{aligned}
&\left(E_{u}^{n+1},\right.\left.A^{-1} E_{u}^{n+1}\right)-\left(E_{u}^{n}, A^{-1} E_{u}^{n}\right) \\
&+\left(E_{u}^{n+1}-E_{u}^{n}, A^{-1}\left(E_{u}^{n+1}-E_{u}^{n}\right)\right) \\
&-2 \nu \Delta t\left(\Delta E_{u}^{n+1}, A^{-1} E_{u}^{n+1}\right) \\
&=-2 \Delta t b\left(E_{u}^{n+1}, u\left(t_{n+1}\right), A^{-1} E_{u}^{n+1}\right) \\
&-2 \Delta t b\left(u^{n+1}, E_{u}^{n+1}, A^{-1} E_{u}^{n+1}\right)+2 \Delta t\left(R_{u}^{n}, A^{-1} E_{u}^{n+1}\right) \\
&-2 k v^{2} \Delta t\left(j E_{\theta}^{n+1}, A^{-1} E_{u}^{n+1}\right) \\
&\left(E_{\theta}^{n+1}, A^{-1} E_{\theta}^{n+1}\right)-\left(E_{\theta}^{n}, A^{-1} E_{\theta}^{n}\right) \\
&+\left(E_{\theta}^{n+1}-E_{\theta}^{n}, A^{-1}\left(E_{\theta}^{n+1}-E_{\theta}^{n}\right)\right) \\
&- 2 \lambda \nu \Delta t\left(\Delta E_{\theta}^{n+1}, A^{-1} E_{\theta}^{n+1}\right)
\end{aligned}
$$

$$
\begin{aligned}
= & -2 \Delta t \bar{b}\left(E_{u}^{n+1}, \theta\left(t_{n+1}\right), A^{-1} E_{\theta}^{n+1}\right) \\
& -2 \Delta t \bar{b}\left(u^{n+1}, E_{\theta}^{n+1}, A^{-1} E_{\theta}^{n+1}\right)+2 \Delta t\left(R_{\theta}^{n}, A^{-1} E_{\theta}^{n+1}\right) .
\end{aligned}
$$

Taking $u=E_{u}^{n+1}$ in (13) and $\theta=E_{\theta}^{n+1}$ in (16). For the terms $-2 \nu \Delta t\left(\Delta E_{u}^{n+1}, A^{-1} E_{u}^{n+1}\right)$ and $-2 \lambda \nu \Delta t\left(\Delta E_{\theta}^{n+1}, A^{-1} E_{\theta}^{n+1}\right)$, we have

$$
\begin{aligned}
-2 v & \Delta t\left(\Delta E_{u}^{n+1}, A^{-1} E_{u}^{n+1}\right) \\
= & 2 v \Delta t\left(E_{u}^{n+1},-\Delta A^{-1} E_{u}^{n+1}\right) \\
= & 2 v \Delta t\left(E_{u}^{n+1}, E_{u}^{n+1}-\nabla q\right)=2 v \Delta t\left\|E_{u}^{n+1}\right\|_{0}^{2}, \\
-2 \lambda \nu \Delta t\left(\Delta E_{\theta}^{n+1}, A^{-1} E_{\theta}^{n+1}\right) & \\
= & 2 \lambda \nu \Delta t\left(E_{\theta}^{n+1},-\Delta A^{-1} E_{\theta}^{n+1}\right) \\
= & 2 \lambda v \Delta t\left(E_{\theta}^{n+1}, E_{\theta}^{n+1}\right)=2 \lambda v \Delta t\left\|E_{\theta}^{n+1}\right\|_{0}^{2} .
\end{aligned}
$$

For the right-hand side terms of (59), we can estimate them as follows:

$$
\begin{aligned}
& \left|2 \Delta t\left(R_{u}^{n}, A^{-1} E_{u}^{n+1}\right)\right| \leq 2 \Delta t\left\|R_{u}^{n}\right\|_{V^{\prime}}\left\|A^{-1} E_{u}^{n+1}\right\|_{V} \\
& =2 \Delta t\left\|R_{u}^{n}\right\|_{V^{\prime}}\left\|E_{u}^{n+1}\right\|_{V^{\prime}} \\
& \leq \Delta t\left\|E_{u}^{n+1}\right\|_{V^{\prime}}^{2}+\Delta t\left\|R_{u}^{n}\right\|_{V^{\prime}}^{2} \\
& \leq \Delta t\left\|E_{u}^{n+1}\right\|_{V^{\prime}}^{2}+C \Delta t^{2} \int_{t_{n}}^{t_{n+1}}\left\|u_{t t}\right\|_{V^{\prime}}^{2} d t \\
& \left|2 \Delta t\left(R_{\theta}^{n}, A^{-1} E_{\theta}^{n+1}\right)\right| \leq 2 \Delta t\left\|R_{\theta}^{n}\right\|_{W^{\prime}}\left\|A^{-1} E_{\theta}^{n+1}\right\|_{W} \\
& =2 \Delta t\left\|R_{\theta}^{n}\right\|_{W^{\prime}}\left\|E_{\theta}^{n+1}\right\|_{W^{\prime}} \\
& \leq \Delta t\left\|E_{\theta}^{n+1}\right\|_{W^{\prime}}^{2}+\Delta t\left\|R_{\theta}^{n}\right\|_{W^{\prime}}^{2} \\
& \leq \Delta t\left\|E_{\theta}^{n+1}\right\|_{W^{\prime}}^{2}+C \Delta t^{2} \int_{t_{n}}^{t_{n+1}}\left\|\theta_{t t}\right\|_{W^{\prime}}^{2} d t \\
& \left|-2 k v^{2}\left(j E_{\theta}^{n+1}, A^{-1} E_{u}^{n+1}\right) \Delta t\right| \\
& \leq 2 k v^{2} \Delta t\left\|E_{\theta}^{n+1}\right\|_{W^{\prime}}\left\|A^{-1} E_{u}^{n+1}\right\|_{V} \\
& =2 k v^{2} \Delta t\left\|E_{\theta}^{n+1}\right\|_{W^{\prime}}\left\|E_{u}^{n+1}\right\|_{V^{\prime}} \\
& \leq k v^{2} \Delta t\left\|E_{\theta}^{n+1}\right\|_{W^{\prime}}^{2}+k v^{2} \Delta t\left\|E_{u}^{n+1}\right\|_{V^{\prime}}^{2} \text {. }
\end{aligned}
$$

For the nonlinear term, using Lemma 3, one finds

$$
\begin{aligned}
& \left|2 \Delta t b\left(E_{u}^{n+1}, u\left(t_{n+1}\right), A^{-1} E_{u}^{n+1}\right)\right| \\
& \quad \leq 2 C_{5} \Delta t\left\|E_{u}^{n+1}\right\|_{0}\left\|A u\left(t_{n+1}\right)\right\|_{0}\left\|\nabla A^{-1} E_{u}^{n+1}\right\|_{0}
\end{aligned}
$$




$$
\begin{aligned}
& \leq 2 C_{5} \Delta t\left\|E_{u}^{n+1}\right\|_{0}\left\|A u\left(t_{n+1}\right)\right\|_{0}\left\|E_{u}^{n+1}\right\|_{V^{\prime}} \\
& \leq \frac{2 C_{5}^{2}}{v} \Delta t\left\|A u\left(t_{n+1}\right)\right\|_{0}^{2}\left\|E_{u}^{n+1}\right\|_{V^{\prime}}^{2}+\frac{\nu \Delta t}{2}\left\|E_{u}^{n+1}\right\|_{0}^{2}, \\
& \left|b\left(u^{n+1}, E_{u}^{n+1}, A^{-1} E_{u}^{n+1}\right)\right| \\
& =\left|b\left(u\left(t_{n+1}\right), E_{u}^{n+1}, A^{-1} E_{u}^{n+1}\right)-b\left(E_{u}^{n+1}, E_{u}^{n+1}, A^{-1} E_{u}^{n+1}\right)\right| \text {, } \\
& \left|2 \Delta t b\left(u\left(t_{n+1}\right), E_{u}^{n+1}, A^{-1} E_{u}^{n+1}\right)\right| \\
& \leq 2 C_{5} \Delta t\left\|A u\left(t_{n+1}\right)\right\|_{0}\left\|E_{u}^{n+1}\right\|_{0}\left\|\nabla A^{-1} E_{u}^{n+1}\right\|_{0} \\
& \leq 2 C_{5} \Delta t\left\|A u\left(t_{n+1}\right)\right\|_{0}\left\|E_{u}^{n+1}\right\|_{0}\left\|E_{u}^{n+1}\right\|_{V^{\prime}} \\
& \leq \frac{2 C_{5}^{2}}{v} \Delta t\left\|A u\left(t_{n+1}\right)\right\|_{0}^{2}\left\|E_{u}^{n+1}\right\|_{V^{\prime}}^{2}+\frac{\nu \Delta t}{2}\left\|E_{u}^{n+1}\right\|_{0}^{2}, \\
& \left|2 \Delta t b\left(E_{u}^{n+1}, E_{u}^{n+1}, A^{-1} E_{u}^{n+1}\right)\right| \\
& \leq 2 C_{5} \Delta t\left\|E_{u}^{n+1}\right\|_{0}\left\|\nabla E_{u}^{n+1}\right\|_{0}\left\|A A^{-1} E_{u}^{n+1}\right\|_{0} \\
& \leq C \Delta t^{3 / 2}\left\|\nabla E_{u}^{n+1}\right\|_{0}\left\|E_{u}^{n+1}\right\|_{0} \\
& \leq C \Delta t^{2}\left\|\nabla E_{u}^{n+1}\right\|_{0}^{2}+\frac{\nu}{2} \Delta t\left\|E_{u}^{n+1}\right\|_{0}^{2}, \\
& \left|2 \Delta t \bar{b}\left(E_{u}^{n+1}, \theta\left(t_{n+1}\right), A^{-1} E_{\theta}^{n+1}\right)\right| \\
& \leq 2 C_{6} \Delta t\left\|E_{u}^{n+1}\right\|_{0}\left\|\nabla \theta\left(t_{n+1}\right)\right\|_{0}\left\|A A^{-1} E_{\theta}^{n+1}\right\|_{0} \\
& \leq 2 C_{6} \Delta t\left\|E_{u}^{n+1}\right\|_{0}\left\|\nabla \theta\left(t_{n+1}\right)\right\|_{0}\left\|E_{\theta}^{n+1}\right\|_{0} \\
& \leq \frac{2 C_{6}^{2}}{\lambda \nu} \Delta t\left\|\nabla \theta\left(t_{n+1}\right)\right\|_{0}^{2}\left\|E_{u}^{n+1}\right\|_{0}^{2}+\frac{\lambda \nu}{2} \Delta t\left\|E_{\theta}^{n+1}\right\|_{0}^{2}, \\
& \left|\bar{b}\left(u^{n+1}, E_{\theta}^{n+1}, A^{-1} E_{\theta}^{n+1}\right)\right| \\
& =\left|\bar{b}\left(u\left(t_{n+1}\right), E_{\theta}^{n+1}, A^{-1} E_{\theta}^{n+1}\right)-\bar{b}\left(E_{u}^{n+1}, E_{\theta}^{n+1}, A^{-1} E_{\theta}^{n+1}\right)\right|, \\
& \left|2 \Delta t \bar{b}\left(u\left(t_{n+1}\right), E_{\theta}^{n+1}, A^{-1} E_{\theta}^{n+1}\right)\right| \\
& \leq 2 C_{6} \Delta t\left\|A u\left(t_{n+1}\right)\right\|_{0}\left\|E_{\theta}^{n+1}\right\|_{0}\left\|\nabla A^{-1} E_{\theta}^{n+1}\right\|_{0} \\
& \leq 2 C_{6} \Delta t\left\|A u\left(t_{n+1}\right)\right\|_{0}\left\|E_{\theta}^{n+1}\right\|_{0}\left\|E_{\theta}^{n+1}\right\|_{W^{\prime}} \\
& \leq \frac{2 C_{6}^{2}}{\lambda \nu} \Delta t\left\|A u\left(t_{n+1}\right)\right\|_{0}^{2}\left\|E_{\theta}^{n+1}\right\|_{W^{\prime}}^{2}+\frac{\lambda \nu}{2} \Delta t\left\|E_{\theta}^{n+1}\right\|_{0}^{2}, \\
& \left|2 \Delta t \bar{b}\left(E_{u}^{n+1}, E_{\theta}^{n+1}, A^{-1} E_{\theta}^{n+1}\right)\right| \\
& \leq 2 C_{6} \Delta t\left\|E_{u}^{n+1}\right\|_{0}\left\|\nabla E_{\theta}^{n+1}\right\|_{0}\left\|A A^{-1} E_{\theta}^{n+1}\right\|_{0} \\
& \leq C \Delta t^{3 / 2}\left\|\nabla E_{\theta}^{n+1}\right\|_{0}\left\|E_{\theta}^{n+1}\right\|_{0} \\
& \leq C(\lambda \nu) \Delta t^{2}\left\|\nabla E_{\theta}^{n+1}\right\|_{0}^{2}+\frac{\lambda \nu}{2} \Delta t\left\|E_{\theta}^{n+1}\right\|_{0}^{2} .
\end{aligned}
$$

Combining above inequalities with (59) and adding them from $n=0$ to $J$, one finds

$$
\begin{aligned}
\left\|E_{\theta}^{J+1}\right\|_{W^{\prime}}^{2} & +\sum_{n=0}^{J}\left\|E_{\theta}^{n+1}-E_{\theta}^{n}\right\|_{W^{\prime}}^{2}+\frac{\lambda \nu \Delta t}{2} \sum_{n=0}^{J}\left\|E_{\theta}^{n+1}\right\|_{0}^{2} \\
\leq & \Delta t \sum_{n=0}^{J}\left(1+\frac{2 C_{6}^{2}}{\lambda \nu}\left\|A u\left(t_{n+1}\right)\right\|_{0}^{2}\right)\left\|E_{\theta}^{n+1}\right\|_{W^{\prime}}^{2} \\
& +C \Delta t^{2} \int_{0}^{T}\left\|\theta_{t t}\right\|_{W^{\prime}}^{2} d t+C(\lambda \nu) \Delta t \cdot \Delta t \sum_{n=0}^{J}\left\|\nabla E_{\theta}^{n+1}\right\|_{0}^{2} \\
& +\frac{2 C_{6}^{2}}{\lambda \nu} \Delta t \sum_{n=0}^{J}\left\|E_{u}^{n+1}\right\|_{0}^{2}\left\|\nabla \theta\left(t_{n+1}\right)\right\|_{0}^{2}, \\
\left\|E_{u}^{J+1}\right\|_{V^{\prime}}^{2} & +\sum_{n=0}^{J}\left\|E_{u}^{n+1}-E_{u}^{n}\right\|_{V^{\prime}}^{2}+\frac{v \Delta t}{2} \sum_{n=0}^{J}\left\|E_{u}^{n+1}\right\|_{0}^{2} \\
\leq & \Delta t \sum_{n=0}^{J}\left(1+k v^{2}+\frac{4 C_{5}^{2}}{\nu}\left\|A u\left(t_{n+1}\right)\right\|_{0}^{2}\right)\left\|E_{u}^{n+1}\right\|_{V^{\prime}}^{2} \\
& +C \Delta t^{2} \int_{0}^{T}\left\|u_{t t}\right\|_{V^{\prime}}^{2} d t+C \Delta t \cdot \Delta t \sum_{n=0}^{J}\left\|\nabla E_{u}^{n+1}\right\|_{0}^{2} \\
& +k v^{2} \Delta t \sum_{n=0}^{J}\left\|E_{\theta}^{n+1}\right\|_{W^{\prime}}^{2} .
\end{aligned}
$$

Thanks to Lemmas 1, 4 and Theorem 2, we derive that

$$
\begin{aligned}
\left\|E_{\theta}^{J+1}\right\|_{W^{\prime}}^{2} & +\sum_{n=0}^{J}\left\|E_{\theta}^{n+1}-E_{\theta}^{n}\right\|_{W^{\prime}}^{2}+\frac{\lambda \nu \Delta t}{2} \sum_{n=0}^{J}\left\|E_{\theta}^{n+1}\right\|_{0}^{2} \\
\leq & \exp \left(\Delta t \sum_{n=0}^{J}\left(1+\frac{2 C_{6}^{2}}{\lambda \nu}\left\|A u\left(t_{n+1}\right)\right\|_{0}^{2}\right)\right) \\
& \times\left\{C \Delta t^{2}+\frac{2 C_{6}^{2}}{\lambda \nu} \Delta t \sum_{n=0}^{J}\left\|\nabla \theta\left(t_{n+1}\right)\right\|_{0}^{2}\left\|E_{u}^{n+1}\right\|_{0}^{2}\right\}, \\
\left\|E_{u}^{J+1}\right\|_{V^{\prime}}^{2} & +\sum_{n=0}^{J}\left\|E_{u}^{n+1}-E_{u}^{n}\right\|_{V^{\prime}}^{2}+\frac{\nu \Delta t}{2} \sum_{n=0}^{J}\left\|E_{u}^{n+1}\right\|_{0}^{2} \\
\leq & \exp \left(\Delta t \sum_{n=0}^{J}\left(1+k \nu^{2}+\frac{4 C_{5}^{2}}{\nu}\left\|A u\left(t_{n+1}\right)\right\|_{0}^{2}\right)\right) \\
\times & \left\{C \Delta t^{2}+k \nu^{2} \Delta t \sum_{n=0}^{J}\left\|E_{\theta}^{n+1}\right\|_{W^{\prime}}^{2}\right\} .
\end{aligned}
$$

Substituting (65) into (64) and using Lemma 1, we complete the proof. 
Lemma 10. Assume that assumptions (A3)-(A6) are valid. Then, for all $J=0,1, \ldots,[T / \Delta t]-1$, one has

$$
\begin{gathered}
\left\|E_{u}^{J+1}\right\|_{0}^{2}+\sum_{n=0}^{J}\left\|E_{u}^{n+1}-E_{u}^{n}\right\|_{0}^{2}+\nu \Delta t \sum_{n=0}^{J}\left\|\nabla E_{u}^{n+1}\right\|_{0}^{2} \leq C \Delta t^{2}, \\
\left\|E_{\theta}^{J+1}\right\|_{0}^{2}+\sum_{n=0}^{J}\left\|E_{\theta}^{n+1}-E_{\theta}^{n}\right\|_{0}^{2}+\lambda \nu \Delta t \sum_{n=0}^{J}\left\|\nabla E_{\theta}^{n+1}\right\|_{0}^{2} \leq C \Delta t^{2} .
\end{gathered}
$$

Proof. Based on Lemma 9, we reestimate the trilinear terms and right-hand side terms of (51) as follows:

$$
\begin{aligned}
\left|2 \Delta t\left(R_{u}^{n}, E_{u}^{n+1}\right)\right| & \leq 2 \Delta t\left\|R_{u}^{n}\right\|_{V^{\prime}}\left\|E_{u}^{n+1}\right\|_{V} \\
& \leq \frac{v \Delta t}{2}\left\|\nabla E_{u}^{n+1}\right\|_{0}^{2}+C \Delta t^{2} \int_{t_{n}}^{t_{n+1}}\left\|u_{t t}\right\|_{V^{\prime}}^{2} d t, \\
\left|2 \Delta t\left(R_{\theta}^{n}, E_{\theta}^{n+1}\right)\right| & \leq 2 \Delta t\left\|R_{\theta}^{n}\right\|_{W^{\prime}}\left\|E_{\theta}^{n+1}\right\|_{W} \\
& \leq \frac{\lambda \nu \Delta t}{2}\left\|\nabla E_{\theta}^{n+1}\right\|_{0}^{2}+C \Delta t^{2} \int_{t_{n}}^{t_{n+1}}\left\|\theta_{t t}\right\|_{W^{\prime}}^{2} d t .
\end{aligned}
$$

Thanks to (67), taking into account (53) and (55) and summing up (51) from $n=0$ to $J$, we have

$$
\begin{aligned}
\left\|E_{\theta}^{J+1}\right\|_{0}^{2} & +\sum_{n=0}^{J}\left\|E_{\theta}^{n+1}-E_{\theta}^{n}\right\|_{0}^{2}+\lambda \nu \Delta t \sum_{n=0}^{J}\left\|\nabla E_{\theta}^{n+1}\right\|_{0}^{2} \\
\leq & \frac{2 C_{6}^{2}}{\lambda \nu} \Delta t \sum_{n=0}^{J}\left\|A \theta\left(t_{n+1}\right)\right\|_{0}^{2}\left\|E_{u}^{n+1}\right\|_{0}^{2} \\
& +C \Delta t^{2} \int_{0}^{T}\left\|\theta_{t t}\right\|_{W^{\prime}}^{2} d t, \\
\left\|E_{u}^{J+1}\right\|_{0}^{2} & +\sum_{n=0}^{J}\left\|E_{u}^{n+1}-E_{u}^{n}\right\|_{0}^{2}+v \Delta t \sum_{n=0}^{J}\left\|\nabla E_{u}^{n+1}\right\|_{0}^{2} \\
\leq & \Delta t \sum_{n=0}^{J}\left(1+\frac{2 C_{5}^{2}}{v}\left\|A u\left(t_{n+1}\right)\right\|_{0}^{2}\right)\left\|E_{u}^{n+1}\right\|_{0}^{2} \\
& +C \Delta t^{2} \int_{0}^{T}\left\|u_{t t}\right\|_{V^{\prime}}^{2} d t+k^{2} v^{4} \Delta t \sum_{n=0}^{J}\left\|E_{\theta}^{n+1}\right\|_{0}^{2} .
\end{aligned}
$$

We obtain the desired results with application of Lemma 1 at above inequalities.

Lemma 11. Under assumptions (A3)-(A6), for all $J=$ $0,1, \ldots,[T / \Delta t]-1$, one has

$$
\begin{aligned}
& \left\|\nabla E_{u}^{J+1}\right\|_{0}^{2}+\sum_{n=0}^{J}\left\|\nabla\left(E_{u}^{n+1}-E_{u}^{n}\right)\right\|_{0}^{2}+\nu \Delta t \sum_{n=0}^{J}\left\|A E_{u}^{n+1}\right\|_{0}^{2} \leq C \Delta t^{2}, \\
& \left\|\nabla E_{\theta}^{J+1}\right\|_{0}^{2}+\sum_{n=0}^{J}\left\|\nabla\left(E_{\theta}^{n+1}-E_{\theta}^{n}\right)\right\|_{0}^{2}+\lambda \nu \Delta t \sum_{n=0}^{J}\left\|A E_{\theta}^{n+1}\right\|_{0}^{2} \leq C \Delta t^{2} .
\end{aligned}
$$

Proof. Taking the inner product of (50) with $2 \Delta t A E_{u}^{n+1}$ and $2 \Delta t A E_{\theta}^{n+1}$, using the fact that $\nabla \cdot E_{u}^{n+1}=0$, we obtain

$$
\begin{aligned}
\left\|\nabla E_{u}^{n+1}\right\|_{0}^{2}-\left\|\nabla E_{u}^{n}\right\|_{0}^{2}+\left\|\nabla\left(E_{u}^{n+1}-E_{u}^{n}\right)\right\|_{0}^{2} \\
+2 \Delta t b\left(E_{u}^{n+1}, u\left(t_{n+1}\right), A E_{u}^{n+1}\right)+2 \nu \Delta t\left\|A E_{u}^{n+1}\right\|_{0}^{2} \\
+2 \Delta t b\left(u^{n+1}, E_{u}^{n+1}, A E_{u}^{n+1}\right) \\
=-2 k v^{2} \Delta t\left(j E_{\theta}^{n+1}, A E_{u}^{n+1}\right)+2 \Delta t\left(R_{u}^{n}, A E_{u}^{n+1}\right), \\
\left\|\nabla E_{\theta}^{n+1}\right\|_{0}^{2}-\left\|\nabla E_{\theta}^{n}\right\|_{0}^{2}+\left\|\nabla\left(E_{\theta}^{n+1}-E_{\theta}^{n}\right)\right\|_{0}^{2}+2 \lambda \nu \Delta t\left\|A E_{\theta}^{n+1}\right\|_{0}^{2} \\
+2 \Delta t \bar{b}\left(u^{n+1}, E_{\theta}^{n+1}, A E_{\theta}^{n+1}\right) \\
+2 \Delta t \bar{b}\left(E_{u}^{n+1}, \theta\left(t_{n+1}\right), A E_{\theta}^{n+1}\right)=2 \Delta t\left(R_{\theta}^{n}, A E_{\theta}^{n+1}\right) .
\end{aligned}
$$

We treat the right-hand side terms of (70) as follows:

$$
\begin{aligned}
\mid 2 \Delta t & \left(R_{u}^{n}, A E_{u}^{n+1}\right) \mid \\
& \leq 2 \Delta t\left\|R_{u}^{n}\right\|_{0}\left\|A E_{u}^{n+1}\right\|_{0} \\
& \leq C \Delta t^{2} \int_{t_{n}}^{t_{n+1}}\left\|u_{t t}\right\|_{0}^{2} d t+\frac{v \Delta t}{2}\left\|A E_{u}^{n+1}\right\|_{0}^{2}, \\
\mid 2 \Delta t & \left(R_{\theta}^{n}, A E_{\theta}^{n+1}\right) \mid \\
& \leq 2 \Delta t\left\|R_{\theta}^{n}\right\|_{0}\left\|A E_{\theta}^{n+1}\right\|_{0} \\
& \leq C \Delta t^{2} \int_{t_{n}}^{t_{n+1}}\left\|\theta_{t t}\right\|_{0}^{2} d t+\frac{\lambda \nu \Delta t}{2}\left\|A E_{\theta}^{n+1}\right\|_{0}^{2}, \\
\left|-2 k v^{2} \Delta t\left(j E_{\theta}^{n+1}, A E_{u}^{n+1}\right)\right| & \leq 2 k \nu^{2} \Delta t\left\|E_{\theta}^{n+1}\right\|_{0}\left\|A E_{u}^{n+1}\right\|_{0} \\
& \leq C \Delta t\left\|E_{\theta}^{n+1}\right\|_{0}^{2}+\frac{v \Delta t}{4}\left\|A E_{u}^{n+1}\right\|_{0}^{2} .
\end{aligned}
$$

By Lemma 3, for the trilinear terms, one finds

$$
\begin{aligned}
\mid 2 \Delta t \bar{b} & \left(E_{u}^{n+1}, \theta\left(t_{n+1}\right), A E_{\theta}^{n+1}\right) \mid \\
& \leq 2 \Delta t C_{6}\left\|A \theta\left(t_{n+1}\right)\right\|_{0}\left\|\nabla E_{u}^{n+1}\right\|_{0}\left\|A E_{\theta}^{n+1}\right\|_{0} \\
& \leq \frac{\lambda \nu}{2} \Delta t\left\|A E_{\theta}^{n+1}\right\|_{0}^{2}+\frac{2 C_{6}^{2}}{\lambda \nu} \Delta t\left\|A \theta\left(t_{n+1}\right)\right\|_{0}^{2}\left\|\nabla E_{u}^{n+1}\right\|_{0}^{2}, \\
\left|2 \Delta t \bar{b}\left(u^{n+1}, E_{\theta}^{n+1}, A E_{\theta}^{n+1}\right)\right| & \leq 2 C_{6} \Delta t\left\|A u^{n+1}\right\|_{0}\left\|\nabla E_{\theta}^{n+1}\right\|_{0}\left\|A E_{\theta}^{n+1}\right\|_{0} \\
\leq & \frac{\lambda \nu}{2} \Delta t\left\|A E_{\theta}^{n+1}\right\|_{0}^{2}+\frac{2 C_{6}^{2}}{\lambda \nu} \Delta t\left\|A u^{n+1}\right\|_{0}^{2}\left\|\nabla E_{\theta}^{n+1}\right\|_{0}^{2},
\end{aligned}
$$




$$
\begin{aligned}
\mid 2 \Delta t b & \left(E_{u}^{n+1}, u\left(t_{n+1}\right), A E_{u}^{n+1}\right) \mid \\
& \leq 2 \Delta t C_{5}\left\|A u\left(t_{n+1}\right)\right\|_{0}\left\|\nabla E_{u}^{n+1}\right\|_{0}\left\|A E_{u}^{n+1}\right\|_{0} \\
& \leq \frac{v}{4} \Delta t\left\|A E_{u}^{n+1}\right\|_{0}^{2}+\frac{4 C_{5}^{2}}{v} \Delta t\left\|A u\left(t_{n+1}\right)\right\|_{0}^{2}\left\|\nabla E_{u}^{n+1}\right\|_{0}^{2}, \\
\mid 2 \Delta t b & \left(u^{n+1}, E_{u}^{n+1}, A E_{u}^{n+1}\right) \mid \\
\leq & 2 C_{5} \Delta t\left\|A u^{n+1}\right\|_{0}\left\|\nabla E_{u}^{n+1}\right\|_{0}\left\|A E_{u}^{n+1}\right\|_{0} \\
\leq & \frac{v}{4} \Delta t\left\|A E_{u}^{n+1}\right\|_{0}^{2}+\frac{4 C_{5}^{2}}{v} \Delta t\left\|A u^{n+1}\right\|_{0}^{2}\left\|\nabla E_{u}^{n+1}\right\|_{0}^{2} .
\end{aligned}
$$

Combining above inequalities with (70) and summing $n$ from 0 to $J$ we obtain

$$
\begin{gathered}
\left\|\nabla E_{\theta}^{J+1}\right\|_{0}^{2}+\sum_{n=0}^{J}\left\|\nabla\left(E_{\theta}^{n+1}-E_{\theta}^{n}\right)\right\|_{0}^{2}+\lambda \nu \Delta t \sum_{n=0}^{J}\left\|A E_{\theta}^{n+1}\right\|_{0}^{2} \\
\leq C \Delta t^{2} \int_{0}^{T}\left\|\theta_{t}\right\|_{0}^{2} d t+\frac{2 C_{6}^{2}}{\lambda \nu} \Delta t \sum_{n=0}^{J}\left\|A \theta\left(t_{n+1}\right)\right\|_{0}^{2}\left\|\nabla E_{u}^{n+1}\right\|_{0}^{2} \\
+\frac{2 C_{6}^{2}}{\lambda \nu} \Delta t \sum_{n=0}^{J}\left\|A u^{n+1}\right\|_{0}^{2}\left\|\nabla E_{\theta}^{n+1}\right\|_{0}^{2}, \\
\left\|\nabla E_{u}^{J+1}\right\|_{0}^{2}+\sum_{n=0}^{J}\left\|\nabla\left(E_{u}^{n+1}-E_{u}^{n}\right)\right\|_{0}^{2}+v \Delta t \sum_{n=0}^{J}\left\|A E_{u}^{n+1}\right\|_{0}^{2} \\
\leq C \Delta t^{2} \int_{0}^{T}\left\|u_{t t}\right\|_{0}^{2} d t+C \Delta t \sum_{n=0}^{J}\left\|E_{\theta}^{n+1}\right\|_{0}^{2} \\
+\frac{2 C_{5}^{2}}{v} \Delta t \sum_{n=0}^{J}\left(\left\|A u\left(t_{n+1}\right)\right\|_{0}^{2}+\left\|A u^{n+1}\right\|_{0}^{2}\right)\left\|\nabla E_{u}^{n+1}\right\|_{0}^{2} .
\end{gathered}
$$

Thanks to Lemmas 10 and 1 we complete the proof.

(b) Error estimates for pressure in scheme (28) are as follows.

Now, we give the estimates for $r^{n+1}=p\left(t_{n+1}\right)-p^{n+1}$ which shows that $p^{n+1}$ is order 1 approximation to $p$ in both $L^{\infty}\left(L^{2}\right)$ and $L^{2}\left(L^{2}\right)$ norms. In order to achieve this aim, we firstly provide some estimates for $d_{t} E_{u}^{n+1}=\left(E_{u}^{n+1}-E_{u}^{n}\right) / \Delta t$ and $d_{t} E_{\theta}^{n+1}=\left(E_{\theta}^{n+1}-E_{\theta}^{n}\right) / \Delta t$.

Lemma 12. Under assumptions (A3)-(A7), for all $J=$ $0,1, \ldots,[T / \Delta t]-1$, one has

$$
\begin{aligned}
& \left\|d_{t} E_{u}^{J+1}\right\|_{0}^{2}+\sum_{n=0}^{J}\left\|d_{t} E_{u}^{n+1}-d_{t} E_{u}^{n}\right\|_{0}^{2}+v \Delta t \sum_{n=0}^{J}\left\|\nabla d_{t} E_{u}^{n+1}\right\|_{0}^{2} \\
& \leq C \Delta t^{2}
\end{aligned}
$$

$$
\begin{aligned}
& \left\|d_{t} E_{\theta}^{J+1}\right\|_{0}^{2}+\sum_{n=0}^{J}\left\|d_{t} E_{\theta}^{n+1}-d_{t} E_{\theta}^{n}\right\|_{0}^{2}+\lambda \nu \Delta t \sum_{n=0}^{J}\left\|\nabla d_{t} E_{\theta}^{n+1}\right\|_{0}^{2} \\
& \leq C \Delta t^{2} .
\end{aligned}
$$

Proof. From problem (50) we obtain that for all $v \in V$ and $\psi \in W$

$$
\begin{aligned}
&\left(d_{t t} E_{u}^{n+1}, v\right)-v\left(\Delta d_{t} E_{u}^{n+1}, v\right) \\
&=\left(d_{t} R_{u}^{n}, v\right)-k v^{2}\left(j d_{t} E_{\theta}^{n+1}, v\right)-b\left(d_{t} E_{u}^{n+1}, u\left(t_{n+1}\right), v\right) \\
&-b\left(E_{u}^{n}, d_{t} u\left(t_{n+1}\right), v\right)-b\left(d_{t} u^{n+1}, E_{u}^{n+1}, v\right) \\
&-b\left(u^{n}, d_{t} E_{u}^{n+1}, v\right), \\
&\left(d_{t t} E_{\theta}^{n+1}, \psi\right)-\lambda \nu\left(\Delta d_{t} E_{\theta}^{n+1}, \psi\right) \\
&=\left(d_{t} R_{\theta}^{n}, \psi\right)-\bar{b}\left(d_{t} E_{u}^{n+1}, \theta\left(t_{n+1}\right), \psi\right) \\
& \quad-\bar{b}\left(E_{u}^{n}, d_{t} \theta\left(t_{n+1}\right), \psi\right)-\bar{b}\left(d_{t} u^{n+1}, E_{\theta}^{n+1}, \psi\right) \\
& \quad-\bar{b}\left(u^{n}, d_{t} E_{\theta}^{n+1}, \psi\right) .
\end{aligned}
$$

Choosing $v=2 \Delta t d_{t} E_{u}^{n+1}$ and $\psi=2 \Delta t d_{t} E_{\theta}^{n+1}$ in (75) we deduce

$$
\begin{aligned}
\left\|d_{t} E_{u}^{n+1}\right\|_{0}^{2}- & \left\|d_{t} E_{u}^{n}\right\|_{0}^{2}+\left\|d_{t} E_{u}^{n+1}-d_{t} E_{u}^{n}\right\|_{0}^{2}+2 v \Delta t\left\|\nabla d_{t} E_{u}^{n+1}\right\|_{0}^{2} \\
=2 \Delta t\{ & \left(d_{t} R_{u}^{n}, d_{t} E_{u}^{n+1}\right)-k v^{2}\left(j d_{t} E_{\theta}^{n+1}, d_{t} E_{u}^{n+1}\right) \\
& -b\left(d_{t} E_{u}^{n+1}, u\left(t_{n+1}\right), d_{t} E_{u}^{n+1}\right) \\
& -b\left(E_{u}^{n}, d_{t} u\left(t_{n+1}\right), d_{t} E_{u}^{n+1}\right) \\
& \left.-b\left(d_{t} u^{n+1}, E_{u}^{n+1}, d_{t} E_{u}^{n+1}\right)\right\}
\end{aligned}
$$

$$
\begin{gathered}
\left\|d_{t} E_{\theta}^{n+1}\right\|_{0}^{2}-\left\|d_{t} E_{\theta}^{n}\right\|_{0}^{2}+\left\|d_{t} E_{\theta}^{n+1}-d_{t} E_{\theta}^{n}\right\|_{0}^{2} \\
+2 \lambda \nu \Delta t\left\|\nabla d_{t} E_{\theta}^{n+1}\right\|_{0}^{2} \\
=2 \Delta t\left\{\left(d_{t} R_{\theta}^{n}, d_{t} E_{\theta}^{n+1}\right)-\bar{b}\left(d_{t} E_{u}^{n+1}, \theta\left(t_{n+1}\right), d_{t} E_{\theta}^{n+1}\right)\right. \\
-\bar{b}\left(E_{u}^{n}, d_{t} \theta\left(t_{n+1}\right), d_{t} E_{\theta}^{n+1}\right) \\
\left.-\bar{b}\left(d_{t} u^{n+1}, E_{\theta}^{n+1}, d_{t} E_{\theta}^{n+1}\right)\right\} .
\end{gathered}
$$


Now, we estimate the right-hand side terms of (76)-(77) separately. For $\left(d_{t} R_{u}^{n}, d_{t} E_{u}^{n+1}\right)$ and $\left(d_{t} R_{\theta}^{n}, d_{t} E_{\theta}^{n+1}\right)$, using the techniques that are adopted by $\mathrm{He}$ in [20], we have

$$
\begin{aligned}
& \left(d_{t} R_{u}^{n}, d_{t} E_{u}^{n+1}\right) \\
& \quad=-\frac{1}{\Delta t^{2}} \int_{t_{n}}^{t_{n+1}}\left(t-t_{n}\right) \int_{t-\Delta t}^{t}\left(u_{t t t}(s), d_{t} E_{u}^{n+1}\right) d s d t, \\
& \left(d_{t} R_{\theta}^{n}, d_{t} E_{\theta}^{n+1}\right) \\
& \quad=-\frac{1}{\Delta t^{2}} \int_{t_{n}}^{t_{n+1}}\left(t-t_{n}\right) \int_{t-\Delta t}^{t}\left(\theta_{t t t}(s), d_{t} E_{\theta}^{n+1}\right) d s d t
\end{aligned}
$$

for all $2 \leq n \leq J$. We deduce from above equalities that

$$
\begin{aligned}
\mid 2 \Delta & t\left(d_{t} R_{u}^{n}, d_{t} E_{u}^{n+1}\right) \mid \\
\leq & 2 \Delta t\left\|d_{t} R_{u}^{n}\right\|_{0}\left\|d_{t} E_{u}^{n+1}\right\|_{0} \\
\leq & C(\nu) \Delta t\left\|d_{t} R_{u}^{n}\right\|_{0}^{2}+\frac{v}{4} \Delta t\left\|\nabla d_{t} E_{u}^{n+1}\right\|_{0}^{2} \\
\leq & c(\nu) \Delta t \\
\quad & \times\left[\Delta t^{-3 / 2}\left(\int_{t_{n}}^{t_{n+1}}\left(t-t_{n}\right)^{2}\left\|\int_{t-\Delta t}^{t} u_{t t t}(s) d s\right\|_{0}^{2} d t\right)^{1 / 2}\right]^{2} \\
& +\frac{v}{4} \Delta t\left\|\nabla d_{t} E_{u}^{n+1}\right\|_{0}^{2} \\
\leq & C \Delta t^{2} \int_{t_{n-1}}^{t_{n+1}}\left\|u_{t t t}\right\|_{0}^{2} d t+\frac{v}{4} \Delta t\left\|\nabla d_{t} E_{u}^{n+1}\right\|_{0}^{2} .
\end{aligned}
$$

In the same way, we obtain

$$
\begin{aligned}
& \left|2 \Delta t\left(d_{t} R_{\theta}^{n}, d_{t} E_{\theta}^{n+1}\right)\right| \\
& \quad \leq C(\lambda \nu) \Delta t^{2} \int_{t_{n-1}}^{t_{n+1}}\left\|\theta_{t t t}\right\|_{0}^{2} d t+\frac{\lambda \nu}{4} \Delta t\left\|\nabla d_{t} E_{\theta}^{n+1}\right\|_{0}^{2} .
\end{aligned}
$$

For the nonlinear terms, with the help of Lemma 3, one finds

$$
\begin{aligned}
\mid 2 \Delta t & \left(d_{t} E_{u}^{n+1}, u\left(t_{n+1}\right), d_{t} E_{u}^{n+1}\right) \mid \\
\leq & 2 C_{5} \Delta t\left\|A u\left(t_{n+1}\right)\right\|_{0}\left\|\nabla d_{t} E_{u}^{n+1}\right\|_{0}\left\|d_{t} E_{u}^{n+1}\right\|_{0} \\
& \leq \frac{v \Delta t}{4}\left\|\nabla d_{t} E_{u}^{n+1}\right\|_{0}^{2}+\frac{4 C_{5}^{2}}{v} \Delta t\left\|A u\left(t_{n+1}\right)\right\|_{0}^{2}\left\|d_{t} E_{u}^{n+1}\right\|_{0}^{2}, \\
\mid 2 \Delta t b & \left(E_{u}^{n}, d_{t} u\left(t_{n+1}\right), d_{t} E_{u}^{n+1}\right) \mid \\
\leq & 2 C_{5} \Delta t\left\|E_{u}^{n}\right\|_{0}\left\|A d_{t} u\left(t_{n+1}\right)\right\|_{0}\left\|\nabla d_{t} E_{u}^{n+1}\right\|_{0} \\
\leq & \frac{v \Delta t}{4}\left\|\nabla d_{t} E_{u}^{n+1}\right\|_{0}^{2}+\frac{4 C_{5}^{2}}{v} \Delta t\left\|A u_{t}\left(t_{n}\right)+\mathcal{O}(\Delta t)\right\|_{0}^{2}\left\|E_{u}^{n}\right\|_{0}^{2},
\end{aligned}
$$

$$
\begin{aligned}
& \left|2 \Delta t b\left(d_{t} u^{n+1}, E_{u}^{n+1}, d_{t} E_{u}^{n+1}\right)\right| \\
& \leq 2 C_{5} \Delta t\left\|A d_{t} u^{n+1}\right\|_{0}\left\|E_{u}^{n+1}\right\|_{0}\left\|\nabla d_{t} E_{u}^{n+1}\right\|_{0} \\
& \leq \frac{v \Delta t}{4}\left\|\nabla d_{t} E_{u}^{n+1}\right\|_{0}^{2}+\frac{4 C_{5}^{2}}{v} \Delta t\left\|A u_{t}^{n}+\mathcal{O}(\Delta t)\right\|_{0}^{2}\left\|E_{u}^{n+1}\right\|_{0}^{2}, \\
& \left|2 \Delta t \bar{b}\left(d_{t} E_{u}^{n+1}, \theta\left(t_{n+1}\right), d_{t} E_{\theta}^{n+1}\right)\right| \\
& \leq 2 \Delta t C_{6}\left\|A \theta\left(t_{n+1}\right)\right\|_{0}\left\|\nabla d_{t} E_{\theta}^{n+1}\right\|_{0}\left\|d_{t} E_{u}^{n+1}\right\|_{0} \\
& \leq \frac{\lambda \nu \Delta t}{4}\left\|\nabla d_{t} E_{\theta}^{n+1}\right\|_{0}^{2}+\frac{4 C_{6}^{2}}{\lambda \nu} \Delta t\left\|A \theta\left(t_{n+1}\right)\right\|_{0}^{2}\left\|d_{t} E_{u}^{n+1}\right\|_{0}^{2}, \\
& \left|2 \Delta t \bar{b}\left(E_{u}^{n}, d_{t} \theta\left(t_{n+1}\right), d_{t} E_{\theta}^{n+1}\right)\right| \\
& \leq 2 C_{6} \Delta t\left\|A d_{t} \theta\left(t_{n+1}\right)\right\|_{0}\left\|\nabla d_{t} E_{\theta}^{n+1}\right\|_{0}\left\|E_{u}^{n}\right\|_{0} \\
& \leq \frac{\lambda \nu \Delta t}{4}\left\|\nabla d_{t} E_{\theta}^{n+1}\right\|_{0}^{2}+\frac{4 C_{6}^{2}}{\lambda \nu} \Delta t\left\|A \theta_{t}\left(t_{n}\right)+\mathcal{O}(\Delta t)\right\|_{0}^{2}\left\|E_{u}^{n}\right\|_{0}^{2}, \\
& \left|2 \Delta t \bar{b}\left(d_{t} u^{n+1}, E_{\theta}^{n+1}, d_{t} E_{\theta}^{n+1}\right)\right| \\
& \leq 2 C_{6} \Delta t\left\|A d_{t} u^{n+1}\right\|_{0}\left\|\nabla d_{t} E_{\theta}^{n+1}\right\|_{0}\left\|E_{\theta}^{n+1}\right\|_{0} \\
& \leq \frac{\lambda \nu \Delta t}{4}\left\|\nabla d_{t} E_{\theta}^{n+1}\right\|_{0}^{2}+\frac{4 C_{6}^{2}}{\lambda \nu} \Delta t\left\|A u_{t}^{n}+\mathcal{O}(\Delta t)\right\|_{0}^{2}\left\|E_{\theta}^{n+1}\right\|_{0}^{2}, \\
& \left|2 k v^{2} \Delta t\left(j d_{t} E_{\theta}^{n+1}, d_{t} E_{u}^{n+1}\right)\right| \\
& \leq k v^{2} \Delta t\left\|d_{t} E_{\theta}^{n+1}\right\|_{0}^{2}+k v^{2} \Delta t\left\|d_{t} E_{u}^{n+1}\right\|_{0}^{2} .
\end{aligned}
$$

Combining above inequalities with (76)-(77) and summing from $n=0$ to $J$, we arrive at

$$
\begin{gathered}
\left\|d_{t} E_{u}^{J+1}\right\|_{0}^{2}+\sum_{n=0}^{J}\left\|d_{t} E_{u}^{n+1}-d_{t} E_{u}^{n}\right\|_{0}^{2}+v \Delta t \sum_{n=0}^{J}\left\|\nabla d_{t} E_{u}^{n+1}\right\|_{0}^{2} \\
\leq C \Delta t^{2} \int_{0}^{T}\left\|u_{t t t}\right\|_{0}^{2} d s+k v^{2} \Delta t \sum_{n=0}^{J}\left\|d_{t} E_{\theta}^{n+1}\right\|_{0}^{2} \\
+\Delta t \sum_{n=0}^{J}\left(\left\|A u\left(t_{n+1}\right)\right\|_{0}^{2}+k v^{2}\right)\left\|d_{t} E_{u}^{n+1}\right\|_{0}^{2} \\
+\frac{4 C_{5}^{2}}{v}\left(\Delta t \sum_{n=0}^{J}\left\|A u_{t}^{n}+\mathcal{O}(\Delta t)\right\|_{0}^{2}\left\|E_{u}^{n+1}\right\|_{0}^{2}\right. \\
\left.+\Delta t \sum_{n=0}^{J}\left\|A u_{t}\left(t_{n}\right)+\mathcal{O}(\Delta t)\right\|_{0}^{2}\left\|E_{u}^{n}\right\|_{0}^{2}\right)
\end{gathered}
$$




$$
\begin{gathered}
\left\|d_{t} E_{\theta}^{J+1}\right\|_{0}^{2}+\sum_{n=0}^{J}\left\|d_{t} E_{\theta}^{n+1}-d_{t} E_{\theta}^{n}\right\|_{0}^{2}+\lambda \nu \Delta t \sum_{n=0}^{J}\left\|\nabla d_{t} E_{\theta}^{n+1}\right\|_{0}^{2} \\
\leq C \Delta t^{2} \int_{0}^{T}\left\|\theta_{t t t}\right\|_{0}^{2} d s+\frac{4 C_{6}^{2}}{\lambda \nu} \Delta t \sum_{n=0}^{J}\left\|A \theta\left(t_{n+1}\right)\right\|_{0}^{2}\left\|d_{t} E_{u}^{n+1}\right\|_{0}^{2} \\
+\frac{4 C_{6}^{2}}{\lambda \nu}\left(\Delta t \sum_{n=0}^{J}\left\|A \theta_{t}\left(t_{n}\right)+\mathcal{O}(\Delta t)\right\|_{0}^{2}\left\|E_{u}^{n}\right\|_{0}^{2}\right. \\
\left.+\Delta t \sum_{n=0}^{J}\left\|A u_{t}^{n}+\mathcal{O}(\Delta t)\right\|_{0}^{2}\left\|E_{\theta}^{n+1}\right\|_{0}^{2}\right) .
\end{gathered}
$$

Substituting (83) into (82) and using Lemma 1, we obtain the desired results.

Remark 13. In the estimates of trilinear terms, we used the bounds of $\left\|A u_{t}^{n+1}\right\|_{0}$ and $\left\|A \theta_{t}^{n+1}\right\|_{0}$ which can be proved by differentiating (12) with respect to time, using the backward Euler scheme to discrete the equations and following the proofs of Lemmas 4 and 5. In the same way, we can also obtain the bounds of $\left\|A u_{t}^{d, n+1}\right\|_{0}$ and $\left\|A \theta_{t}^{d, n+1}\right\|_{0}$ for the decoupled scheme. Here, we omit these proofs for simplification.

Now, we are in the position of deriving the optimal error estimate for pressure in $L^{\infty}\left(L^{2}\right)$ norm based on the results presented in Lemmas 10, 11, and 12.

Theorem 14. Under assumptions (A3)-(A7) for all $J=$ $0,1, \ldots,[T / \Delta t]-1$ one has

$$
\left\|p\left(t_{n+1}\right)-p^{n+1}\right\|_{0} \leq C \Delta t .
$$

Proof. We rewrite the first equation of (50) as follows:

$$
\begin{aligned}
-\nabla E_{p}^{n+1}= & d_{t} E_{u}^{n+1}-v \Delta E_{u}^{n+1}-R_{u}^{n}+k v^{2} j E_{\theta}^{n+1} \\
& +\left(u\left(t_{n+1}\right) \cdot \nabla\right) u\left(t_{n+1}\right)-\left(u^{n+1} \cdot \nabla\right) u^{n+1} .
\end{aligned}
$$

Take the inner product of (85) with an arbitrary $v \in X$ and use Poincare inequality to obtain

$$
\begin{gathered}
\left|\left(d_{t} E_{u}^{n+1}, v\right)\right| \leq\left\|d_{t} E_{u}^{n+1}\right\|_{0}\|v\|_{0} \leq C_{3}\left\|d_{t} E_{u}^{n+1}\right\|_{0}\|\nabla v\|_{0}, \\
\left|v\left(\Delta E_{u}^{n+1}, v\right)\right| \leq v\left\|\nabla E_{u}^{n+1}\right\|_{0}\|\nabla v\|_{0}, \\
\left|\left(R_{u}^{n}, v\right)\right| \leq\left\|R_{u}^{n}\right\|_{0}\|v\|_{0} \leq c \Delta t\left(\int_{t_{n}}^{t_{n+1}} t\left\|u_{t t}\right\|_{0}^{2}\right)^{1 / 2}\|\nabla v\|_{0}, \\
\left|k v^{2}\left(j E_{\theta}^{n+1}, v\right)\right| \leq k v^{2}\left\|E_{\theta}^{n+1}\right\|_{0}\|v\|_{0} \leq C_{3} k v^{2}\left\|E_{\theta}^{n+1}\right\|_{0}\|\nabla v\|_{0} .
\end{gathered}
$$

For the nonlinear terms, use the results provided in Lemma 3 to arrive at

$$
\begin{gathered}
\left|b\left(E_{u}^{n+1}, u\left(t_{n+1}\right), v\right)\right| \leq C_{5}\left\|E_{u}^{n+1}\right\|_{0}\left\|A u\left(t_{n+1}\right)\right\|_{0}\|\nabla v\|_{0}, \\
\left|b\left(u^{n+1}, E_{u}^{n+1}, v\right)\right| \leq C_{5}\left\|A u^{n+1}\right\|_{0}\left\|E_{u}^{n+1}\right\|_{0}\|\nabla v\|_{0} .
\end{gathered}
$$

Thanks to (32), we have

$$
\begin{aligned}
\left\|E_{p}^{n+1}\right\|_{0} \leq C\left(\beta^{-1}\right)\{ & \left\|d_{t} E_{u}^{n+1}\right\|_{0} \\
& +\left(\left\|A u^{n+1}\right\|_{0}+\left\|A u\left(t_{n+1}\right)\right\|_{0}\right)\left\|E_{u}^{n+1}\right\|_{0} \\
& +v\left\|\nabla E_{u}^{n+1}\right\|_{0}+k v^{2}\left\|E_{\theta}^{n+1}\right\|_{0} \\
& \left.+\Delta t\left(\int_{t_{n}}^{t_{n+1}} t\left\|u_{t t}\right\|_{0}^{2} d t\right)^{1 / 2}\right\} .
\end{aligned}
$$

With the results obtained in Lemmas $5,10,11$, and 12 , we complete the proof.

\section{Error Estimates of the Decoupled Numerical Scheme}

In this section, we try to establish the optimal error estimates for the decoupled algorithm (31). We just point out the differences between the coupled and decoupled numerical schemes in the following lemmas. In order to simplify the representation, we denote

$$
\begin{aligned}
E_{\phi}^{d, n} & =\phi\left(t_{n}\right)-\phi^{d, n} \quad(\phi \text { takes } u, p, \theta), \\
z_{\theta}^{n} & =\int_{t_{n}}^{t_{n+1}} \theta_{t} d s, \quad z_{u}^{n}=\int_{t_{n}}^{t_{n+1}} u_{t} d s .
\end{aligned}
$$

Lemma 15. Under assumptions (A3)-(A6), for all $J=$ $0,1, \ldots,[T / \Delta t-1]$, one has

$$
\begin{aligned}
& \left\|E_{u}^{d, J+1}\right\|_{0}^{2}+\sum_{n=0}^{J}\left\|E_{u}^{d, n+1}-E_{u}^{d, n}\right\|_{0}^{2}+\nu \Delta t \sum_{n=0}^{J}\left\|\nabla E_{u}^{d, n+1}\right\|_{0}^{2} \\
& \leq C \Delta t \\
& \left\|E_{\theta}^{d, J+1}\right\|_{0}^{2}+\sum_{n=0}^{J}\left\|E_{\theta}^{d, n+1}-E_{\theta}^{d, n}\right\|_{0}^{2}+\lambda \nu \Delta t \sum_{n=0}^{J}\left\|\nabla E_{\theta}^{d, n+1}\right\|_{0}^{2} \\
& \leq C \Delta t .
\end{aligned}
$$

Proof. By subtracting (29) from (47) we have

$$
\begin{gathered}
\frac{E_{u}^{d, n+1}-E_{u}^{d, n}}{\Delta t}-v \Delta E_{u}^{d, n+1}+\left(u\left(t_{n+1}\right) \cdot \nabla\right) u\left(t_{n+1}\right) \\
-\left(u^{d, n} \cdot \nabla\right) u^{d, n+1}+\nabla E_{p}^{d, n+1} \\
=-k v^{2} j E_{\theta}^{d, n}-k v^{2} j z_{\theta}^{n}+R_{u}^{n}, \\
\nabla \cdot E_{u}^{d, n+1}=0, \\
\frac{E_{\theta}^{d, n+1}-E_{\theta}^{d, n}}{\Delta t}-\lambda \nu \Delta E_{\theta}^{d, n+1}+\left(u\left(t_{n+1}\right) \cdot \nabla\right) \theta\left(t_{n+1}\right) \\
-\left(u^{d, n} \cdot \nabla\right) \theta^{d, n+1}=R_{\theta}^{n} .
\end{gathered}
$$


Taking the inner product of (94) with $2 \Delta t E_{u}^{d, n+1}, 2 \Delta t E_{p}^{d, n+1}$, and $2 \Delta t E_{\theta}^{d, n+1}$, problem (94) can be transformed into

$$
\begin{aligned}
& \| E_{u}^{d, n+1}\left\|_{0}^{2}-\right\| E_{u}^{d, n}\left\|_{0}^{2}+\right\| E_{u}^{d, n+1}-E_{u}^{d, n} \|_{0}^{2} \\
&+2 \nu \Delta t\left\|\nabla E_{u}^{d, n+1}\right\|_{0}^{2}+2 \Delta t b\left(E_{u}^{d, n}, u\left(t_{n+1}\right), E_{u}^{d, n+1}\right) \\
&+2 \Delta t b\left(z_{u}^{n}, u\left(t_{n+1}\right), E_{u}^{d, n+1}\right) \\
&=-2 k \nu^{2} \Delta t\left(j E_{\theta}^{d, n}, E_{u}^{d, n+1}\right)-2 k v^{2} \Delta t\left(j z_{\theta}^{n}, E_{u}^{d, n+1}\right) \\
&+2 \Delta t\left(R_{u}^{n}, E_{u}^{d, n+1}\right), \\
&\left\|E_{\theta}^{d, n+1}\right\|_{0}^{2}-\left\|E_{\theta}^{d, n}\right\|_{0}^{2}+\left\|E_{\theta}^{d, n+1}-E_{\theta}^{d, n}\right\|_{0}^{2} \\
&+2 \Delta t \bar{b}\left(z_{u}^{n}, \theta\left(t_{n+1}\right), E_{\theta}^{d, n+1}\right)+2 \lambda \nu \Delta t\left\|\nabla E_{\theta}^{d, n+1}\right\|_{0}^{2} \\
&+2 \Delta t \bar{b}\left(E_{u}^{d, n}, \theta\left(t_{n+1}\right), E_{\theta}^{d, n+1}\right)=2 \Delta t\left(R_{\theta}^{n}, E_{\theta}^{d, n+1}\right) .
\end{aligned}
$$

Now, we estimate the right-hand side terms of (95) as follows:

$$
\begin{aligned}
& \left|-2 k v^{2} \Delta t\left(j E_{\theta}^{d, n}, E_{u}^{d, n+1}\right)\right| \leq k^{2} \nu^{4} \Delta t\left\|E_{\theta}^{d, n}\right\|_{0}^{2}+\Delta t\left\|E_{u}^{d, n+1}\right\|_{0}^{2}, \\
& \left|-2 k v^{2} \Delta t\left(j z_{\theta}^{n}, E_{u}^{d, n+1}\right)\right| \\
& \leq 2 k v^{2} \Delta t\left\|z_{\theta}^{n}\right\|_{0}\left\|E_{u}^{d, n+1}\right\|_{0} \\
& \leq \frac{v \Delta t}{5}\left\|E_{u}^{d, n+1}\right\|_{0}^{2}+C \Delta t^{2} \int_{t_{n}}^{t_{n+1}}\left\|u_{t}\right\|_{0}^{2} d s .
\end{aligned}
$$

For the nonlinear term, thanks to Lemma 3, we arrive at

$$
\begin{aligned}
\mid 2 \Delta t b & \left(E_{u}^{d, n}, u\left(t_{n+1}\right), E_{u}^{d, n+1}\right) \mid \\
\leq & 2 C_{5} \Delta t\left\|E_{u}^{d, n}\right\|_{0}\left\|A u\left(t_{n+1}\right)\right\|_{0}\left\|\nabla E_{u}^{d, n+1}\right\|_{0} \\
& \leq \frac{5 C_{5}^{2}}{v} \Delta t\left\|E_{u}^{d, n+1}\right\|_{0}^{2}\left\|A u\left(t_{n+1}\right)\right\|_{0}^{2}+\frac{v \Delta t}{5}\left\|\nabla E_{u}^{d, n+1}\right\|_{0}^{2}, \\
\mid 2 \Delta t b & \left(z_{u}^{n}, u\left(t_{n+1}\right), E_{u}^{d, n+1}\right) \mid \\
& \leq 2 C_{5} \Delta t\left\|z_{u}^{n}\right\|_{0}\left\|A u\left(t_{n+1}\right)\right\|_{0}\left\|\nabla E_{u}^{d, n+1}\right\|_{0} \\
& \leq C \Delta t^{2} \int_{t_{n}}^{t_{n+1}}\left\|u_{t}\right\|_{0}^{2} d t+\frac{v \Delta t}{5}\left\|\nabla E_{u}^{d, n+1}\right\|_{0}^{2}, \\
\mid 2 \Delta t \bar{b} & \left(E_{u}^{d, n}, \theta\left(t_{n+1}\right), E_{\theta}^{n+1}\right) \mid \\
\leq & 2 C_{6} \Delta t\left\|E_{u}^{d, n}\right\|_{0}\left\|A \theta\left(t_{n+1}\right)\right\|_{0}\left\|\nabla E_{\theta}^{d, n+1}\right\|_{0} \\
& \leq \frac{5 C_{6}^{2}}{\lambda \nu} \Delta t\left\|E_{u}^{d, n}\right\|_{0}^{2}\left\|A \theta\left(t_{n+1}\right)\right\|_{0}^{2}+\frac{\lambda \nu}{5} \Delta t\left\|_{\theta}^{d, n+1}\right\|_{0}^{2},
\end{aligned}
$$

$$
\begin{aligned}
& \left|2 \Delta t \bar{b}\left(z_{u}^{n}, \theta\left(t_{n+1}\right), E_{\theta}^{d, n+1}\right)\right| \\
& \quad \leq 2 C_{5} \Delta t\left\|z_{u}^{n}\right\|_{0}\left\|A \theta\left(t_{n+1}\right)\right\|_{0}\left\|\nabla E_{\theta}^{d, n+1}\right\|_{0} \\
& \quad \leq C \Delta t^{2} \int_{t_{n}}^{t_{n+1}}\left\|u_{t}\right\|_{0}^{2} d t+\frac{\lambda v \Delta t}{5}\left\|\nabla E_{\theta}^{d, n+1}\right\|_{0}^{2} .
\end{aligned}
$$

Combining above inequalities with (52) and (53) and summing from $n=0$ to $J$ one finds

$$
\begin{gathered}
\left\|E_{\theta}^{d, J+1}\right\|_{0}^{2}+\sum_{n=0}^{J}\left\|E_{\theta}^{d, n+1}-E_{\theta}^{d, n}\right\|_{0}^{2}+\lambda \nu \Delta t \sum_{n=0}^{J}\left\|\nabla E_{\theta}^{d, n+1}\right\|_{0}^{2} \\
\leq C\left\{\Delta t \sum_{n=0}^{J}\left\|E_{u}^{d, n}\right\|_{0}^{2}+\Delta t \int_{0}^{T} t\left\|\theta_{t t}\right\|_{0}^{2} d s\right. \\
\left.+\Delta t^{2} \int_{0}^{T}\left\|u_{t}\right\|_{0}^{2} d s\right\}, \\
\left\|E_{u}^{d, J+1}\right\|_{0}^{2}+\sum_{n=0}^{J}\left\|E_{u}^{d, n+1}-E_{u}^{d, n}\right\|_{0}^{2}+v \Delta t \sum_{n=0}^{J}\left\|\nabla E_{u}^{d, n+1}\right\|_{0}^{2} \\
\leq C\left\{\begin{array}{c}
\Delta t \sum_{n=0}^{J}\left\|E_{u}^{d, n}\right\|_{0}^{2}+\Delta t \int_{0}^{T} t\left\|u_{t t}\right\|_{0}^{2} \\
\left.+\Delta t \sum_{n=0}^{J}\left\|E_{\theta}^{d, n}\right\|_{0}^{2}+\Delta t^{2} \int_{0}^{T}\left\|u_{t}\right\|_{0}^{2} d t\right\} .
\end{array}\right.
\end{gathered}
$$

We complete the proof by using Lemma 1 and (22).

Lemma 16. For all $J=0,1, \ldots,[T / \Delta t]-1$, under assumptions (A3)-(A6), one has

$$
\begin{aligned}
& \left\|E_{u}^{d, J+1}\right\|_{V^{\prime}}^{2}+\sum_{n=0}^{m}\left\|E_{u}^{d, n+1}-E_{u}^{d, n}\right\|_{V^{\prime}}^{2}+\frac{\nu \Delta t}{2} \sum_{n=0}^{J}\left\|E_{u}^{d, n+1}\right\|_{0}^{2} \\
& \leq C \Delta t^{2}, \\
& \left\|E_{\theta}^{d, J+1}\right\|_{W^{\prime}}^{2}+\sum_{n=0}^{m}\left\|E_{\theta}^{d, n+1}-E_{\theta}^{d, n}\right\|_{W^{\prime}}^{2}+\frac{\lambda \nu \Delta t}{2} \sum_{n=0}^{J}\left\|E_{\theta}^{d, n+1}\right\|_{0}^{2} \\
& \leq C \Delta t^{2} .
\end{aligned}
$$

Proof. Taking the inner product of (94) with $2 \Delta t A^{-1} E_{u}^{d, n+1}$ and $2 \Delta t A^{-1} E_{\theta}^{d, n+1}$, using the fact that $\nabla \cdot E_{u}^{d, n+1}=0$ and the self-adjointness of $A^{-1}$, we get

$$
\begin{aligned}
\left(E_{u}^{d, n+1}\right. & \left., A^{-1} E_{u}^{d, n+1}\right)-\left(E_{u}^{d, n}, A^{-1} E_{u}^{d, n}\right) \\
& +\left(E_{u}^{d, n+1}-E_{u}^{d, n}, A^{-1}\left(E_{u}^{d, n+1}-E_{u}^{d, n}\right)\right) \\
& -2 \nu \Delta t\left(\Delta E_{u}^{d, n+1}, A^{-1} E_{u}^{d, n+1}\right)
\end{aligned}
$$




$$
\begin{aligned}
&=-2 \Delta t b\left(z_{u}^{n}, u\left(t_{n+1}\right), A^{-1} E_{u}^{d, n+1}\right)+2 \Delta t\left(R_{u}^{n}, A^{-1} E_{u}^{d, n+1}\right) \\
&-2 \Delta t b\left(u^{d, n}, E_{u}^{d, n+1}, A^{-1} E_{u}^{d, n+1}\right) \\
&-2 \Delta t b\left(E_{u}^{d n}, u\left(t_{n+1}\right), A^{-1} E_{u}^{d, n+1}\right) \\
&-2 k v^{2} \Delta t\left(j E_{\theta}^{d, n}, A^{-1} E_{u}^{d, n+1}\right)-2 k v^{2} \Delta t\left(j z_{\theta}^{n}, A^{-1} E_{u}^{d, n+1}\right), \\
&\left(E_{\theta}^{d, n+1}, A^{-1} E_{\theta}^{d, n+1}\right)-\left(E_{\theta}^{d, n}, A^{-1} E_{\theta}^{d, n}\right) \\
&+\left(E_{\theta}^{d, n+1}-E_{\theta}^{d, n}, A^{-1}\left(E_{\theta}^{d, n+1}-E_{\theta}^{d, n}\right)\right) \\
&-2 \lambda v \Delta t\left(\Delta E_{\theta}^{d, n+1}, A^{-1} E_{\theta}^{d, n+1}\right) \\
&=-2 \Delta t \bar{b}\left(E_{u}^{d, n}, \theta\left(t_{n+1}\right), A^{-1} E_{\theta}^{d, n+1}\right)+2 \Delta t\left(R_{\theta}^{n}, A^{-1} E_{\theta}^{d, n+1}\right) \\
&-2 \Delta t \bar{b}\left(u^{d, n}, E_{\theta}^{d, n+1}, A^{-1} E_{\theta}^{d, n+1}\right) \\
&-2 \Delta t \bar{b}\left(z_{u}^{n}, \theta\left(t_{n+1}\right), A^{-1} E_{\theta}^{d, n+1}\right) .
\end{aligned}
$$

For the right-hand side terms of (100), we have

$$
\begin{aligned}
&\left|-2 k v^{2}\left(j E_{\theta}^{d, n}, A^{-1} E_{u}^{d, n+1}\right) \Delta t\right| \\
& \leq C \Delta t\left\|E_{\theta}^{d, n}\right\|_{W^{\prime}}\left\|A^{-1} E_{u}^{d, n+1}\right\|_{V} \\
& \leq C \Delta t\left\|E_{\theta}^{d, n}\right\|_{W^{\prime}}\left\|E_{u}^{d, n+1}\right\|_{V^{\prime}} \\
& \leq C \Delta t\left\|E_{\theta}^{d, n}\right\|_{W^{\prime}}^{2}+C \Delta t\left\|E_{u}^{d, n+1}\right\|_{V^{\prime}}, \\
& \mid 2 \Delta t \bar{b}\left(E_{u}^{d, n}, \theta\left(t_{n+1}\right), A^{-1} E_{\theta}^{d, n+1}\right) \mid \\
& \leq C \Delta t\left\|E_{u}^{d, n}\right\|_{0}\left\|\nabla \theta\left(t_{n+1}\right)\right\|_{0}\left\|A A^{-1} E_{\theta}^{d, n+1}\right\|_{0} \\
& \leq C \Delta t\left\|E_{u}^{d, n}\right\|_{0}\left\|\nabla \theta\left(t_{n+1}\right)\right\|_{0}\left\|E_{\theta}^{d, n+1}\right\|_{0} \\
& \leq C \Delta t\left\|\nabla \theta\left(t_{n+1}\right)\right\|_{0}^{2}\left\|E_{u}^{d, n}\right\|_{W^{\prime}}^{2}+\frac{\lambda v \Delta t}{2}\left\|E_{\theta}^{d, n+1}\right\|_{0}^{2}, \\
&\left|\bar{b}\left(u^{d, n}, E_{\theta}^{d, n+1}, A^{-1} E_{\theta}^{d, n+1}\right)\right| \\
&=\mid \bar{b}\left(u\left(t_{n}\right), E_{\theta}^{d, n+1}, A^{-1} E_{\theta}^{d, n+1}\right) \\
& \quad-\bar{b}\left(E_{u}^{d, n}, E_{\theta}^{d, n+1}, A^{-1} E_{\theta}^{d, n+1}\right) \mid, \\
& \leq C \Delta t\left\|E_{u}^{d, n}\right\|_{0}\left\|\nabla E_{\theta}^{d, n+1}\right\|_{0}\left\|A A^{-1} E_{\theta}^{d, n+1}\right\|_{0} \\
&\left|2 \Delta t \bar{b}\left(u\left(t_{n}\right), E_{\theta}^{d, n+1}, A^{-1} E_{\theta}^{d, n+1}\right)\right| \\
& \leq C \Delta t\left\|A u\left(t_{n}\right)\right\|_{0}\left\|E_{\theta}^{d, n+1}\right\|_{0}\left\|\nabla A^{-1} E_{\theta}^{d, n+1}\right\|_{0} \\
& \leq C \Delta t\left\|A u\left(t_{n}\right)\right\|_{0}\left\|E_{\theta}^{d, n+1}\right\|_{0}\left\|E_{\theta}^{d, n+1}\right\|_{W^{\prime}} \\
&\left|2 \Delta t \bar{b}\left(E_{u}^{d, n}, E_{\theta}^{d, n+1}, A^{-1} E_{\theta}^{d, n+1}\right)\right| \\
& \leq A u\left(t_{n}\right)\left\|_{0}^{2}\right\| E_{\theta}^{d, n+1}\left\|_{W^{\prime}}^{2}+\frac{\lambda v \Delta t}{2}\right\| E_{\theta}^{d, n+1} \|_{0}^{2}, \\
& \leq
\end{aligned}
$$

$$
\begin{aligned}
& \leq C \Delta t^{3 / 2}\left\|\nabla E_{\theta}^{d, n+1}\right\|_{0}\left\|E_{\theta}^{d, n+1}\right\|_{0} \\
& \leq C(\lambda \nu) \Delta t^{2}\left\|\nabla E_{\theta}^{d, n+1}\right\|_{0}^{2}+\frac{\lambda \nu}{2} \Delta t\left\|E_{\theta}^{d, n+1}\right\|_{0}^{2}, \\
\left|2 \Delta t \bar{b}\left(z_{u}^{n}, \theta\left(t_{n+1}\right), A^{-1} E_{\theta}^{d, n+1}\right)\right|^{2} & \leq 2 C_{6} \Delta t\left\|z_{u}^{n}\right\|_{0}\left\|A \theta\left(t_{n+1}\right)\right\|_{0}\left\|\nabla A^{-1} E_{\theta}^{d, n+1}\right\|_{0} \\
\leq & 2 C_{6} \Delta t\left\|z_{u}^{n}\right\|_{0}\left\|A \theta\left(t_{n+1}\right)\right\|_{0}\left\|E_{\theta}^{d, n+1}\right\|_{W^{\prime}} \\
& \leq C\left\|A \theta\left(t_{n+1}\right)\right\|_{0}^{2}\left\|E_{\theta}^{d, n+1}\right\|_{W^{\prime}}^{2}+C \Delta t^{2} \int_{t_{n}}^{t_{n+1}}\left\|u_{t}\right\|_{0}^{2} d t,
\end{aligned}
$$$$
\left|2 \Delta t b\left(E_{u}^{d, n}, u\left(t_{n+1}\right), A^{-1} E_{u}^{d, n+1}\right)\right|
$$$$
\leq C \Delta t\left\|E_{u}^{d, n}\right\|_{0}\left\|A u\left(t_{n+1}\right)\right\|_{0}\left\|\nabla A^{-1} E_{u}^{d, n+1}\right\|_{0}
$$$$
\leq\left\|E_{u}^{d, n}\right\|_{0}\left\|A u\left(t_{n+1}\right)\right\|_{0}\left\|E_{u}^{d, n+1}\right\|_{V^{\prime}}
$$$$
\leq C \Delta t\left\|A u\left(t_{n+1}\right)\right\|_{0}^{2}\left\|E_{\theta}^{d, n+1}\right\|_{V^{\prime}}^{2}+\frac{\nu \Delta t}{2}\left\|E_{u}^{d, n}\right\|_{0}^{2},
$$$$
\left|b\left(u^{d, n}, E_{u}^{d, n+1}, A^{-1} E_{u}^{d, n+1}\right)\right|
$$$$
=\mid b\left(u\left(t_{n}\right), E_{u}^{d, n+1}, A^{-1} E_{u}^{d, n+1}\right)
$$$$
-b\left(E_{u}^{d, n}, E_{u}^{d, n+1}, A^{-1} E_{u}^{d, n+1}\right) \mid,
$$$$
\left|2 \Delta t b\left(u\left(t_{n}\right), E_{u}^{d, n+1}, A^{-1} E_{u}^{d, n+1}\right)\right|
$$$$
\leq 2 C_{5} \Delta t\left\|A u\left(t_{n}\right)\right\|_{0}\left\|E_{u}^{d, n+1}\right\|_{0}\left\|\nabla A^{-1} E_{u}^{d, n+1}\right\|_{0}
$$$$
\leq 2 C_{5} \Delta t\left\|A u\left(t_{n}\right)\right\|_{0}\left\|E_{u}^{d, n+1}\right\|_{0}\left\|E_{u}^{d, n+1}\right\|_{V^{\prime}}
$$$$
\leq C(\nu) \Delta t\left\|A u\left(t_{n}\right)\right\|_{0}^{2}\left\|E_{u}^{d, n+1}\right\|_{V^{\prime}}^{2}+\frac{\nu \Delta t}{2}\left\|E_{u}^{d, n+1}\right\|_{0}^{2},
$$$$
\left|2 \Delta t b\left(E_{u}^{d, n}, E_{u}^{d, n+1}, A^{-1} E_{u}^{d, n+1}\right)\right|
$$$$
\leq 2 C_{5} \Delta t\left\|E_{u}^{d, n}\right\|_{0}\left\|\nabla E_{u}^{d, n+1}\right\|_{0}\left\|A A^{-1} E_{u}^{d, n+1}\right\|_{0}
$$$$
\leq C \Delta t^{3 / 2}\left\|\nabla E_{u}^{d, n+1}\right\|_{0}\left\|E_{u}^{d, n+1}\right\|_{0}
$$$$
\leq C(\nu) \Delta t^{2}\left\|\nabla E_{u}^{d, n+1}\right\|_{0}^{2}+\frac{\nu \Delta t}{2}\left\|E_{u}^{d, n+1}\right\|_{0}^{2},
$$$$
\left|2 \Delta t b\left(z_{u}^{n}, u\left(t_{n+1}\right), A^{-1} E_{u}^{d, n+1}\right)\right|
$$$$
\leq 2 C_{5} \Delta t\left\|z_{u}^{n}\right\|_{0}\left\|A u\left(t_{n+1}\right)\right\|_{0}\left\|\nabla A^{-1} E_{u}^{d, n+1}\right\|_{0}
$$$$
\leq 2 C_{5} \Delta t\left\|z_{u}^{n}\right\|_{0}\left\|A u\left(t_{n+1}\right)\right\|_{0}\left\|E_{u}^{d, n+1}\right\|_{V^{\prime}}
$$$$
\leq C\left\|A u\left(t_{n+1}\right)\right\|_{0}^{2}\left\|E_{u}^{d, n+1}\right\|_{V^{\prime}}^{2}+C \Delta t^{2} \int_{t_{n}}^{t_{n+1}}\left\|u_{t}\right\|_{0}^{2} d t .
$$ 
For the other terms, we can treat them as we have done in Lemma 9. By combining above inequalities and adding (100) from $n=0$ to $J$, one finds

$$
\begin{aligned}
& \left\|E_{\theta}^{d, J+1}\right\|_{W^{\prime}}^{2}+\sum_{n=0}^{J}\left\|E_{\theta}^{d, n+1}-E_{\theta}^{d, n}\right\|_{W^{\prime}}^{2}+\frac{\lambda \nu \Delta t}{2} \sum_{n=0}^{J}\left\|E_{\theta}^{d, n+1}\right\|_{0}^{2} \\
& \leq C\left\{\Delta t \sum_{n=0}^{J}\left\|E_{\theta}^{d, n+1}\right\|_{W^{\prime}}^{2}+\Delta t^{2} \int_{0}^{T}\left\|\theta_{t t}\right\|_{W^{\prime}}^{2} d t\right. \\
& +\Delta t^{2} \int_{0}^{T}\left\|u_{t}\right\|_{0}^{2} d t+\Delta t \sum_{n=0}^{J}\left\|E_{u}^{d, n}\right\|_{0}^{2}\left\|\nabla \theta\left(t_{n+1}\right)\right\|_{0}^{2} \\
& \left.+\Delta t \sum_{n=0}^{J}\left\|\nabla E_{\theta}^{d, n+1}\right\|_{0}^{2} \Delta t\right\} \\
& \left\|E_{u}^{d, J+1}\right\|_{V^{\prime}}^{2}+\sum_{n=0}^{J}\left\|E_{u}^{d, n+1}-E_{u}^{d, n}\right\|_{V^{\prime}}^{2}+\frac{\nu \Delta t}{2} \sum_{n=0}^{J}\left\|E_{u}^{d, n+1}\right\|_{0}^{2} \\
& \leq C\left\{\Delta t \sum_{n=0}^{J}\left\|E_{u}^{d, n+1}\right\|_{V^{\prime}}^{2}+\Delta t^{2} \int_{0}^{T}\left\|u_{t t}\right\|_{V^{\prime}}^{2} d t\right. \\
& +\Delta t^{2} \int_{0}^{T}\left\|u_{t}\right\|_{0}^{2} d t+\Delta t \sum_{n=0}^{J}\left\|\nabla E_{u}^{d, n+1}\right\|_{0}^{2} \Delta t \\
& \left.+\Delta t \sum_{n=0}^{J}\left\|E_{\theta}^{d, n+1}\right\|_{W^{\prime}}^{2}\right\} \text {. }
\end{aligned}
$$

Applying Lemmas 1, 15 and assumptions (A3)-(A7) we finish the proof.

Lemma 17. Under conditions (A3)-(A6), for all $J=$ $0,1, \ldots,[T / \Delta t]-1$, one has

$$
\begin{aligned}
& \left\|E_{u}^{d, J+1}\right\|_{0}^{2}+\sum_{n=0}^{J}\left\|E_{u}^{d, n+1}-E_{u}^{d, n}\right\|_{0}^{2}+\nu \Delta t \sum_{n=0}^{J}\left\|\nabla E_{u}^{d, n+1}\right\|_{0}^{2} \\
& \leq C \Delta t^{2} \\
& \left\|E_{\theta}^{d, J+1}\right\|_{0}^{2}+\sum_{n=0}^{J}\left\|E_{\theta}^{d, n+1}-E_{\theta}^{d, n}\right\|_{0}^{2}+\lambda \nu \Delta t \sum_{n=0}^{J}\left\|\nabla E_{\theta}^{d, n+1}\right\|_{0}^{2} \\
& \leq C \Delta t^{2} .
\end{aligned}
$$

Proof. Taking the inner product of (94) with $2 \Delta t E_{u}^{d, n+1}$ and $2 \Delta t E_{\theta}^{d, n+1}$, using the fact that $\nabla \cdot E_{u}^{d, n+1}=0$, we obtain (95). Based on (67), and the estimates presented in Lemma 7.
Taking these inequalities of Lemmas 7 and 9 into (95), summing (95) from $n=0$ to $J$, we arrive at

$$
\begin{gathered}
\left\|E_{\theta}^{d, J+1}\right\|_{0}^{2}+\sum_{n=0}^{J}\left\|E_{\theta}^{d, n+1}-E_{\theta}^{d, n}\right\|_{0}^{2}+\lambda \nu \Delta t \sum_{n=0}^{J}\left\|\nabla E_{\theta}^{d, n+1}\right\|_{0}^{2} \\
\leq C\left\{\Delta t \sum_{n=0}^{J}\left\|E_{u}^{d, n}\right\|_{0}^{2}+\Delta t^{2} \int_{0}^{T}\left\|\theta_{t t}\right\|_{W^{\prime}}^{2} d s\right. \\
\left.+\Delta t^{2} \int_{0}^{T}\left\|u_{t}\right\|_{0}^{2} d s\right\}, \\
\left\|E_{u}^{d, J+1}\right\|_{0}^{2}+\sum_{n=0}^{J}\left\|E_{u}^{d, n+1}-E_{u}^{d, n}\right\|_{0}^{2}+v \Delta t \sum_{n=0}^{J}\left\|\nabla E_{u}^{d, n+1}\right\|_{0}^{2} \\
\leq C\left\{\begin{array}{c}
\Delta t \sum_{n=0}^{J}\left\|E_{u}^{d, n}\right\|_{0}^{2}+\Delta t^{2} \int_{0}^{T}\left\|u_{t t}\right\|_{V^{\prime}}^{2} d s \\
\left.+k^{2} v^{4} \Delta t \sum_{n=0}^{J}\left\|E_{\theta}^{d, n+1}\right\|_{0}^{2}+\Delta t^{2} \int_{0}^{T}\left\|u_{t}\right\|_{0}^{2} d s\right\} .
\end{array}\right.
\end{gathered}
$$

We obtain the desired results with application of Lemma 1 at above inequalities.

Lemma 18. Under assumptions (A3)-(A6), for all $J=$ $0,1, \ldots,[T / \Delta t]-1$, one has

$$
\begin{aligned}
& \left\|\nabla E_{u}^{d, J+1}\right\|_{0}^{2}+\sum_{n=0}^{J}\left\|\nabla\left(E_{u}^{d, n+1}-E_{u}^{d, n}\right)\right\|_{0}^{2}+\nu \Delta t \sum_{n=0}^{J}\left\|A E_{u}^{d, n+1}\right\|_{0}^{2} \\
& \leq C \Delta t^{2}, \\
& \left\|\nabla E_{\theta}^{d, J+1}\right\|_{0}^{2}+\sum_{n=0}^{J}\left\|\nabla\left(E_{\theta}^{d, n+1}-E_{\theta}^{d, n}\right)\right\|_{0}^{2}+\lambda \nu \Delta t \sum_{n=0}^{J}\left\|A E_{\theta}^{d, n+1}\right\|_{0}^{2} \\
& \leq C \Delta t^{2} .
\end{aligned}
$$

Proof. Taking the inner product of (94) with $2 \Delta t A E_{u}^{d, n+1}$ and $2 \Delta t A E_{\theta}^{d, n+1}$, using the fact that $\nabla \cdot E_{u}^{d, n+1}=0$, we get

$$
\begin{aligned}
\left\|\nabla E_{u}^{d, n+1}\right\|_{0}^{2}-\left\|\nabla E_{u}^{d, n}\right\|_{0}^{2}+\left\|\nabla\left(E_{u}^{d, n+1}-E_{u}^{d, n}\right)\right\|_{0}^{2} \\
\quad+2 \Delta t b\left(E_{u}^{d, n}, u\left(t_{n+1}\right), A E_{u}^{d, n+1}\right)+2 \nu \Delta t\left\|A E_{u}^{d, n+1}\right\|_{0}^{2} \\
\quad+2 \Delta t b\left(u^{d, n}, E_{u}^{d, n+1}, A E_{u}^{d, n+1}\right) \\
\quad+2 \Delta t b\left(z_{u}^{n}, u\left(t_{n+1}\right), A E_{u}^{d, n+1}\right) \\
=-2 k v^{2} \Delta t\left(j E_{\theta}^{d, n}, A E_{u}^{d, n+1}\right)-2 k v^{2} \Delta t\left(j z_{\theta}^{n}, A E_{u}^{d, n+1}\right) \\
\quad+2 \Delta t\left(R_{u}^{n}, A E_{u}^{d, n+1}\right),
\end{aligned}
$$




$$
\begin{aligned}
& \left\|\nabla E_{\theta}^{d, n+1}\right\|_{0}^{2}-\left\|\nabla E_{\theta}^{d, n}\right\|_{0}^{2}+\left\|\nabla\left(E_{\theta}^{d, n+1}-E_{\theta}^{d, n}\right)\right\|_{0}^{2} \\
& \quad+2 \lambda \nu \Delta t\left\|A E_{\theta}^{d, n+1}\right\|_{0}^{2}+2 \Delta t \bar{b}\left(u^{n}, E_{\theta}^{d, n+1}, A E_{\theta}^{d, n+1}\right) \\
& \quad+2 \Delta t \bar{b}\left(z_{u}^{n}, \theta\left(t_{n+1}\right), A E_{\theta}^{d, n+1}\right) \\
& \quad+2 \Delta t \bar{b}\left(E_{u}^{d, n}, \theta\left(t_{n+1}\right), A E_{\theta}^{d, n+1}\right)=2 \Delta t\left(R_{\theta}^{n}, A E_{\theta}^{d, n+1}\right) .
\end{aligned}
$$

For the trilinear terms, using Lemma 3, we have

$$
\begin{aligned}
& \left|2 \Delta t \bar{b}\left(E_{u}^{d, n}, \theta\left(t_{n+1}\right), A E_{\theta}^{d, n+1}\right)\right| \\
& \leq 2 \Delta t C_{6}\left\|A \theta\left(t_{n+1}\right)\right\|_{0}\left\|\nabla E_{u}^{d, n}\right\|_{0}\left\|A E_{\theta}^{d, n+1}\right\|_{0} \\
& \leq \frac{\lambda \nu}{2} \Delta t\left\|A E_{\theta}^{d, n+1}\right\|_{0}^{2}+\frac{2 C_{6}^{2}}{\lambda \nu} \Delta t\left\|A \theta\left(t_{n+1}\right)\right\|_{0}^{2}\left\|\nabla E_{u}^{d, n}\right\|_{0}^{2}, \\
& \left|2 \Delta t \bar{b}\left(u^{d, n}, E_{\theta}^{d, n+1}, A E_{\theta}^{d, n+1}\right)\right| \\
& \leq 2 \Delta t C_{6}^{2}\left\|A u^{d, n}\right\|_{0}\left\|\nabla E_{\theta}^{d, n+1}\right\|_{0}\left\|A E_{\theta}^{d, n+1}\right\|_{0} \\
& \leq \frac{\lambda \nu}{2} \Delta t\left\|A E_{\theta}^{d, n+1}\right\|_{0}^{2}+\frac{2 C_{6}^{2}}{\lambda \nu} \Delta t\left\|A u^{d, n}\right\|_{0}^{2}\left\|\nabla E_{\theta}^{d, n+1}\right\|_{0}^{2} \text {, } \\
& \left|2 \Delta t \bar{b}\left(z_{u}^{n}, \theta\left(t_{n+1}\right), A E_{\theta}^{d, n+1}\right)\right| \\
& \leq 2 \Delta t C_{6}\left\|A \theta\left(t_{n+1}\right)\right\|_{0}\left\|\nabla z_{u}^{n}\right\|_{0}\left\|A E_{\theta}^{d, n+1}\right\|_{0} \\
& \leq \frac{\lambda \nu}{2} \Delta t\left\|A E_{\theta}^{d, n+1}\right\|_{0}^{2} \\
& +C(\lambda \nu)\left\|A \theta\left(t_{n+1}\right)\right\|_{0}^{2} \Delta t^{2} \int_{t_{n}}^{t_{n+1}} t\left\|\nabla u_{t}\right\|_{0}^{2} d s, \\
& \left|2 \Delta t b\left(E_{u}^{d, n}, u\left(t_{n+1}\right), A E_{u}^{d, n+1}\right)\right| \\
& \leq 2 \Delta t C_{5}\left\|A u\left(t_{n+1}\right)\right\|_{0}\left\|\nabla E_{u}^{d, n}\right\|_{0}\left\|A E_{u}^{d, n+1}\right\|_{0} \\
& \leq \frac{\nu}{4} \Delta t\left\|A E_{u}^{d, n+1}\right\|_{0}^{2}+\frac{4 C_{5}^{2}}{\nu} \Delta t\left\|A u\left(t_{n+1}\right)\right\|_{0}^{2}\left\|\nabla E_{u}^{d, n}\right\|_{0}^{2}, \\
& \left|2 \Delta t b\left(u^{d, n}, E_{u}^{d, n+1}, A E_{u}^{d, n+1}\right)\right| \\
& \leq 2 \Delta t C_{5}^{2}\left\|A u^{d, n}\right\|_{0}\left\|\nabla E_{u}^{d, n+1}\right\|_{0}\left\|A E_{u}^{d, n+1}\right\|_{0} \\
& \leq \frac{v}{4} \Delta t\left\|A E_{u}^{d, n+1}\right\|_{0}^{2}+\frac{4 C_{5}^{2}}{v} \Delta t\left\|A u^{d, n}\right\|_{0}^{2}\left\|\nabla E_{u}^{d, n+1}\right\|_{0}^{2}, \\
& \left|2 \Delta t b\left(z_{u}^{n}, u\left(t_{n+1}\right), A E_{u}^{d, n+1}\right)\right| \\
& \leq 2 \Delta t C_{5}\left\|A u\left(t_{n+1}\right)\right\|_{0}\left\|\nabla z_{u}^{n}\right\|_{0}\left\|A E_{u}^{d, n+1}\right\|_{0} \\
& \leq \frac{\nu}{2} \Delta t\left\|A E_{u}^{d, n+1}\right\|_{0}^{2}+C(\nu)\left\|A u\left(t_{n+1}\right)\right\|_{0}^{2} \Delta t^{2} \int_{t_{n}}^{t_{n+1}} t\left\|\nabla u_{t}\right\|_{0}^{2} d s .
\end{aligned}
$$

Combining above inequalities with (106) and summing $n$ from 0 to $J$ we obtain

$$
\begin{aligned}
& \left\|\nabla E_{\theta}^{d, J+1}\right\|_{0}^{2}+\sum_{n=0}^{J}\left\|\nabla\left(E_{\theta}^{d, n+1}-E_{\theta}^{d, n}\right)\right\|_{0}^{2}+\frac{\lambda \nu}{2} \Delta t \sum_{n=0}^{J}\left\|A E_{\theta}^{d, n+1}\right\|_{0}^{2} \\
& \leq C \Delta t^{2} \int_{0}^{T}\left(\left\|\theta_{t t}\right\|_{0}^{2}+\left\|\nabla u_{t}\right\|_{0}^{2}\right) d t \\
& +\frac{2 C_{6}^{2}}{\lambda \nu}\left\{\Delta t \sum_{n=0}^{J}\left\|A \theta\left(t_{n+1}\right)\right\|_{0}^{2}\left\|\nabla E_{u}^{d, n}\right\|_{0}^{2}\right. \\
& \left.+\Delta t \sum_{n=0}^{m}\left\|A u^{n}\right\|_{0}^{2}\left\|\nabla E_{\theta}^{d, n+1}\right\|_{0}^{2}\right\} \\
& \left\|\nabla E_{u}^{d, J+1}\right\|_{0}^{2}+\sum_{n=0}^{J}\left\|\nabla\left(E_{u}^{d, n+1}-E_{u}^{d, n}\right)\right\|_{0}^{2}+v \Delta t \sum_{n=0}^{J}\left\|A E_{u}^{d, n+1}\right\|_{0}^{2} \\
& \leq C \Delta t^{2} \int_{0}^{T}\left(\left\|u_{t t}\right\|_{0}^{2}+\left\|\nabla u_{t}\right\|_{0}^{2}\right) d t+C \Delta t \sum_{n=0}^{J}\left\|E_{\theta}^{d, n+1}\right\|_{0}^{2} \\
& +\frac{4 C_{5}^{2}}{v}\left\{\Delta t \sum_{n=0}^{J}\left\|A u^{d, n}\right\|_{0}^{2}\left\|\nabla E_{u}^{d, n+1}\right\|_{0}^{2}\right. \\
& \left.+\Delta t \sum_{n=0}^{J}\left\|A u\left(t_{n+1}\right)\right\|_{0}^{2}\left\|\nabla E_{u}^{d, n}\right\|_{0}^{2}\right\}
\end{aligned}
$$

Thanks to Lemmas 1, 6, and 17 we complete the proof.

Lemma 19. Under assumptions (A3)-(A7), for all $J=$ $0,1, \ldots,[T / \Delta t]-1$, one has

$$
\begin{array}{r}
\left\|d_{t} E_{u}^{d, J+1}\right\|_{0}^{2}+\sum_{n=0}^{J}\left\|d_{t} E_{u}^{d, n+1}-d_{t} E_{u}^{d, n}\right\|_{0}^{2} \\
+\nu \Delta t \sum_{n=0}^{J}\left\|\nabla d_{t} E_{u}^{d, n+1}\right\|_{0}^{2} \leq C \Delta t^{2}, \\
\left\|d_{t} E_{\theta}^{d, J+1}\right\|_{0}^{2}+\sum_{n=0}^{J}\left\|d_{t} E_{\theta}^{d, n+1}-d_{t} E_{\theta}^{d, n}\right\|_{0}^{2} \\
+\lambda \nu \Delta t \sum_{n=0}^{J}\left\|\nabla d_{t} E_{\theta}^{d, n+1}\right\|_{0}^{2} \leq C \Delta t^{2} .
\end{array}
$$

Proof. From problem (94) we can obtain that for all $v \in V$ and $\psi \in W$

$$
\begin{aligned}
\left(d_{t t} E_{u}^{d, n+1}, v\right)-v\left(\Delta d_{t} E_{u}^{d, n+1}, v\right) \\
=\left(d_{t} R_{u}^{n}, v\right)-k v^{2}\left(j d_{t} E_{\theta}^{d, n}, v\right)-b\left(d_{t} E_{u}^{d, n}, u\left(t_{n+1}\right), v\right) \\
\quad-b\left(E_{u}^{d, n-1}, d_{t} u\left(t_{n+1}\right), v\right)-b\left(d_{t} u^{d, n}, E_{u}^{d, n+1}, v\right) \\
\quad-b\left(u^{d, n-1}, d_{t} E_{u}^{d, n+1}, v\right)-k v^{2}\left(j d_{t} z_{\theta}^{n}, v\right)
\end{aligned}
$$




$$
\begin{aligned}
& -b\left(d_{t} z_{u}^{n}, u\left(t_{n+1}\right), v\right)-b\left(z_{u}^{n-1}, d_{t} u\left(t_{n+1}\right), v\right), \\
\left(d_{t t} E_{\theta}^{d, n+1}, \psi\right)-\lambda \nu\left(\Delta d_{t} E_{\theta}^{d, n+1}, \psi\right) & =\left(d_{t} R_{\theta}^{n}, \psi\right)-\bar{b}\left(d_{t} E_{u}^{d, n}, \theta\left(t_{n+1}\right), \psi\right) \\
& -\bar{b}\left(E_{u}^{d, n-1}, d_{t} \theta\left(t_{n+1}\right), \psi\right)-\bar{b}\left(d_{t} u^{d, n}, E_{\theta}^{d, n+1}, \psi\right) \\
& -\bar{b}\left(u^{d, n-1}, d_{t} E_{\theta}^{d, n+1}, \psi\right)-\bar{b}\left(d_{t} z_{u}^{n}, \theta\left(t_{n+1}\right), \psi\right) \\
& -\bar{b}\left(z_{u}^{n-1}, d_{t} \theta\left(t_{n+1}\right), \psi\right) .
\end{aligned}
$$

Choosing $v=2 \Delta t d_{t} E_{u}^{d, n+1}$ and $\psi=2 \Delta t d_{t} E_{\theta}^{d n+1}$ in (110) one gets

$$
\begin{aligned}
\left\|d_{t} E_{u}^{d, n+1}\right\|_{0}^{2} & -\left\|d_{t} E_{u}^{d, n}\right\|_{0}^{2}+\left\|d_{t} E_{u}^{d, n+1}-d_{t} E_{u}^{d, n}\right\|_{0}^{2} \\
+v \Delta t & \left\|\nabla d_{t} E_{u}^{d, n+1}\right\|_{0}^{2} \\
=2 \Delta t\{ & \left(d_{t} R_{u}^{n}, d_{t} E_{u}^{d, n+1}\right)-k \nu^{2}\left(j d_{t} E_{\theta}^{d, n}, d_{t} E_{u}^{d, n+1}\right) \\
& -b\left(d_{t} E_{u}^{d, n}, u\left(t_{n+1}\right), d_{t} E_{u}^{d, n+1}\right) \\
& -b\left(E_{u}^{d, n-1}, d_{t} u\left(t_{n+1}\right), d_{t} E_{u}^{d, n+1}\right) \\
& -b\left(d_{t} u^{d, n}, E_{u}^{d, n+1}, d_{t} E_{u}^{d, n+1}\right) \\
& -k v^{2}\left(j d_{t} z_{\theta}^{n}, d_{t} E_{u}^{d, n+1}\right) \\
& -b\left(d_{t} z_{u}^{n}, u\left(t_{n+1}\right), d_{t} E_{u}^{d, n+1}\right) \\
& \left.-b\left(z_{u}^{n-1}, d_{t} u\left(t_{n+1}\right), d_{t} E_{u}^{d, n+1}\right)\right\}, \\
\left\|d_{t} E_{\theta}^{d, n+1}\right\|_{0}^{2} & -\left\|d_{t} E_{\theta}^{d, n}\right\|_{0}^{2}+\left\|d_{t} E_{\theta}^{d, n+1}-d_{t} E_{\theta}^{d, n}\right\|_{0}^{2} \\
+2 \lambda \nu \Delta t & \nabla d_{t} E_{\theta}^{d, n+1} \|_{0}^{2} \\
=2 \Delta t & \left\{\left(d_{t} R_{\theta}^{d, n}, d_{t} E_{\theta}^{d, n+1}\right)-\bar{b}\left(d_{t} E_{u}^{d, n}, \theta\left(t_{n+1}\right), d_{t} E_{\theta}^{d, n+1}\right)\right. \\
& -\bar{b}\left(E_{u}^{d, n-1}, d_{t} \theta\left(t_{n+1}\right), d_{t} E_{\theta}^{d, n+1}\right) \\
& -\bar{b}\left(d_{t} u^{d, n}, E_{\theta}^{d, n+1}, d_{t} E_{\theta}^{d, n+1}\right) \\
& -\bar{b}\left(d_{t} z_{u}^{n}, \theta\left(t_{n+1}\right), d_{t} E_{\theta}^{d, n+1}\right) \\
& \left.-\bar{b}\left(z_{u}^{n-1}, d_{t} \theta\left(t_{n+1}\right), d_{t} E_{\theta}^{d, n+1}\right)\right\} .
\end{aligned}
$$

For the nonlinear terms, thanks to Lemma 3, we deduce that

$$
\begin{aligned}
& \left|2 \Delta t b\left(d_{t} E_{u}^{d, n}, u\left(t_{n+1}\right), d_{t} E_{u}^{d, n+1}\right)\right| \\
& \leq 2 \Delta t C_{5}\left\|A u\left(t_{n+1}\right)\right\|_{0}\left\|\nabla d_{t} E_{u}^{d, n+1}\right\|_{0}\left\|d_{t} E_{u}^{d, n}\right\|_{0} \\
& \leq \frac{v \Delta t}{4}\left\|\nabla d_{t} E_{u}^{d, n+1}\right\|_{0}^{2}+C \Delta t\left\|A u\left(t_{n+1}\right)\right\|_{0}^{2}\left\|d_{t} E_{u}^{d, n}\right\|_{0}^{2},
\end{aligned}
$$

$$
\begin{aligned}
& \left|2 \Delta t b\left(E_{u}^{d, n-1}, d_{t} u\left(t_{n+1}\right), d_{t} E_{u}^{d, n+1}\right)\right| \\
& \leq 2 \Delta t C_{5}\left\|A d_{t} u\left(t_{n+1}\right)\right\|_{0}\left\|\nabla d_{t} E_{u}^{d, n+1}\right\|_{0}\left\|E_{u}^{d, n-1}\right\|_{0} \\
& \leq \frac{v \Delta t}{4}\left\|\nabla d_{t} E_{u}^{d, n+1}\right\|_{0}^{2}+C \Delta t\left\|A u_{t}\left(t_{n}\right)+\mathcal{O}(\Delta t)\right\|_{0}^{2}\left\|E_{u}^{d, n-1}\right\|_{0}^{2}, \\
& \left|2 \Delta t b\left(d_{t} u^{d, n}, E_{u}^{d, n+1}, d_{t} E_{u}^{d, n+1}\right)\right| \\
& \leq 2 \Delta t C_{5}\left\|A d_{t} u^{d, n}\right\|_{0}\left\|\nabla d_{t} E_{u}^{d, n+1}\right\|_{0}\left\|E_{u}^{d, n+1}\right\|_{0} \\
& \leq \frac{\nu \Delta t}{4}\left\|\nabla d_{t} E_{u}^{d, n+1}\right\|_{0}^{2}+C \Delta t\left\|A u_{t}^{n-1}+\mathcal{O}(\Delta t)\right\|_{0}^{2}\left\|E_{u}^{d, n+1}\right\|_{0}^{2}, \\
& \left|2 \Delta t b\left(d_{t} z_{u}^{n}, u\left(t_{n+1}\right), d_{t} E_{u}^{d, n+1}\right)\right| \\
& \leq 2 C_{5} \Delta t\left\|A u\left(t_{n+1}\right)\right\|_{0}\left\|\nabla d_{t} E_{u}^{d, n+1}\right\|_{0}\left\|d_{t} z_{u}^{n}\right\|_{0} \\
& \leq 2 C_{5} \Delta t^{2}\left\|A u\left(t_{n+1}\right)\right\|_{0}\left\|\nabla d_{t} E_{u}^{d, n+1}\right\|_{0}\left\|u_{t t}\left(t_{n}\right)+\mathcal{O}\left(\Delta t^{2}\right)\right\|_{0} \\
& \leq \frac{\nu \Delta t}{4}\left\|\nabla d_{t} E_{u}^{d, n+1}\right\|_{0}^{2} \\
& +C \Delta t^{3}\left\|u_{t t}\left(t_{n}\right)+\mathcal{O}\left(\Delta t^{2}\right)\right\|_{0}^{2}\left\|A u\left(t_{n+1}\right)\right\|_{0}^{2}, \\
& \left|2 \Delta t b\left(z_{u}^{n-1}, d_{t} u\left(t_{n+1}\right), d_{t} E_{u}^{d, n+1}\right)\right| \\
& \leq 2 C_{5} \Delta t\left\|A d_{t} u\left(t_{n+1}\right)\right\|_{0}\left\|\nabla d_{t} E_{u}^{d, n+1}\right\|_{0}\left\|z_{u}^{n}\right\|_{0} \\
& \leq \frac{v \Delta t}{4}\left\|\nabla d_{t} E_{u}^{d, n+1}\right\|_{0}^{2} \\
& +C \Delta t^{2}\left\|A u_{t}\left(t_{n}\right)+\mathcal{O}(\Delta t)\right\|_{0}^{2} \int_{t_{n-2}}^{t_{n-1}}\left\|u_{t}\right\|_{0}^{2} d t,
\end{aligned}
$$

$$
\begin{aligned}
& \left|2 \Delta t \bar{b}\left(d_{t} E_{u}^{d, n}, \theta\left(t_{n+1}\right), d_{t} E_{\theta}^{d, n+1}\right)\right| \\
& \leq 2 \Delta t C_{6}\left\|A \theta\left(t_{n+1}\right)\right\|_{0}\left\|\nabla d_{t} E_{\theta}^{n+1}\right\|_{0}\left\|d t E_{u}^{d, n}\right\|_{0} \\
& \leq \frac{\lambda \nu \Delta t}{4}\left\|\nabla d_{t} E_{\theta}^{d, n+1}\right\|_{0}^{2}+C \Delta t\left\|A \theta\left(t_{n+1}\right)\right\|_{0}^{2}\left\|d_{t} E_{u}^{d, n}\right\|_{0}^{2}, \\
& \left|2 \Delta t \bar{b}\left(E_{u}^{d, n-1}, d_{t} \theta\left(t_{n+1}\right), d_{t} E_{\theta}^{d, n+1}\right)\right| \\
& \leq 2 \Delta t C_{6}\left\|A d_{t} \theta\left(t_{n+1}\right)\right\|_{0}\left\|\nabla d_{t} E_{\theta}^{d, n+1}\right\|_{0}\left\|E_{u}^{d, n-1}\right\|_{0} \\
& \leq \frac{\lambda \nu \Delta t}{4}\left\|\nabla d_{t} E_{\theta}^{d, n+1}\right\|_{0}^{2}+C \Delta t\left\|A \theta_{t}\left(t_{n}\right)+\mathcal{O}(\Delta t)\right\|_{0}^{2}\left\|E_{u}^{d, n-1}\right\|_{0}^{2}, \\
& \left|2 \Delta t \bar{b}\left(d_{t} u^{d, n}, E_{\theta}^{d, n+1}, d_{t} E_{\theta}^{d, n+1}\right)\right| \\
& \leq 2 \Delta t C_{6}\left\|A d_{t} u^{d, n}\right\|_{0}\left\|\nabla d_{t} E_{\theta}^{d, n+1}\right\|_{0}\left\|E_{\theta}^{d, n+1}\right\|_{0} \\
& \leq \frac{\lambda \nu \Delta t}{4}\left\|\nabla d_{t} E_{\theta}^{d, n+1}\right\|_{0}^{2}+C \Delta t\left\|A u_{t}^{n-1}+\mathcal{O}(\Delta t)\right\|_{0}^{2}\left\|E_{\theta}^{d, n+1}\right\|_{0}^{2}, \\
& \left|2 k \nu^{2} \Delta t\left(j d_{t} E_{\theta}^{d, n+1}, d_{t} E_{u}^{d, n+1}\right)\right| \\
& \quad \leq k \nu^{2} \Delta t\left\|d_{t} E_{\theta}^{d, n+1}\right\|_{0}^{2}+k \nu^{2} \Delta t\left\|d_{t} E_{u}^{d, n+1}\right\|_{0}^{2},
\end{aligned}
$$




$$
\begin{aligned}
\mid 2 \Delta t \bar{b} & \left(d_{t} z_{u}^{n}, \theta\left(t_{n+1}\right), d_{t} E_{\theta}^{d, n+1}\right) \mid \\
\leq & 2 C_{6} \Delta t\left\|A \theta\left(t_{n+1}\right)\right\|_{0}\left\|\nabla d_{t} E_{\theta}^{d, n+1}\right\|_{0}\left\|d_{t} z_{u}^{n}\right\|_{0} \\
\leq & \frac{\lambda \nu \Delta t}{4}\left\|\nabla d_{t} E_{\theta}^{d, n+1}\right\|_{0}^{2} \\
& +C \Delta t^{3}\left\|u_{t t}\left(t_{n}\right)+\mathcal{O}\left(\Delta t^{2}\right)\right\|_{0}^{2}\left\|A \theta\left(t_{n+1}\right)\right\|_{0}^{2}, \\
\left|2 \Delta t \bar{b}\left(z_{u}^{n-1}, d_{t} \theta\left(t_{n+1}\right), d_{t} E_{\theta}^{d, n+1}\right)\right| & \\
\leq & 2 C_{6} \Delta t\left\|A d_{t} \theta\left(t_{n+1}\right)\right\|_{0}\left\|\nabla d_{t} E_{\theta}^{d, n+1}\right\|_{0}\left\|z_{u}^{n}\right\|_{0} \\
\leq & \frac{\lambda \nu \Delta t}{4}\left\|\nabla d_{t} E_{\theta}^{d, n+1}\right\|_{0}^{2} \\
& +C \Delta t^{2}\left\|A \theta_{t}\left(t_{n}\right)+\mathcal{O}(\Delta t)\right\|_{0}^{2} \int_{t_{n-2}}^{t_{n-1}}\left\|u_{t}\right\|_{0}^{2} d t .
\end{aligned}
$$

Combine above inequalities with (79)-(80) with (111), summing them from $n=0$ to $J$, to arrive at

$$
\begin{aligned}
& \left\|d_{t} E_{u}^{d, J+1}\right\|_{0}^{2}+\sum_{n=0}^{J}\left\|d_{t} E_{u}^{d, n+1}-d_{t} E_{u}^{d, n}\right\|_{0}^{2}+v \Delta t \sum_{n=0}^{J}\left\|\nabla d_{t} E_{u}^{d, n+1}\right\|_{0}^{2} \\
& =C\left\{\Delta t^{2} \int_{0}^{T}\left\|u_{t t t}\right\|_{0}^{2} d s+\Delta t \sum_{n=0}^{J}\left\|d_{t} E_{\theta}^{d, n+1}\right\|_{0}^{2}\right. \\
& +\Delta t \sum_{n=0}^{J}\left\|A u\left(t_{n+1}\right)\right\|_{0}^{2}\left\|d_{t} E_{u}^{d, n+1}\right\|_{0}^{2}+\Delta t \int_{0}^{T}\left\|u_{t}\right\|_{0}^{2} d t \\
& +\Delta t \sum_{n=0}^{J}\left(\left\|A u_{t}\left(t_{n}\right)+\mathcal{O}(\Delta t)\right\|_{0}^{2}\right. \\
& \left.+\left\|A u_{t}^{d, n-1}+\mathcal{O}(\Delta t)\right\|_{0}^{2}\right)\left\|E_{u}^{d, n+1}\right\|_{0}^{2} \\
& \left.+\Delta t^{2}\left\|A u\left(t_{n+1}\right)\right\|_{0}^{2}\left\|u_{t t}\right\|_{0}^{2}\right\} \text {, } \\
& \left\|d_{t} E_{\theta}^{d, J+1}\right\|_{0}^{2}+\sum_{n=0}^{J}\left\|d_{t} E_{\theta}^{d, n+1}-d_{t} E_{\theta}^{d, n}\right\|_{0}^{2} \\
& +\lambda v \Delta t \sum_{n=0}^{J}\left\|\nabla d_{t} E_{\theta}^{d, n+1}\right\|_{0}^{2} \\
& =C\left\{\Delta t^{2} \int_{0}^{T}\left\|\theta_{t t t}\right\|_{0}^{2} d s+\Delta t \sum_{n=0}^{J}\left\|A \theta\left(t_{n+1}\right)\right\|_{0}^{2}\left\|d_{t} E_{u}^{d, n}\right\|_{0}^{2}\right. \\
& +\Delta t \int_{0}^{T}\left\|u_{t}\right\|_{0}^{2} d t+\Delta t \sum_{n=0}^{J}\left\|A \theta_{t}\left(t_{n}\right)+\mathcal{O}(\Delta t)\right\|_{0}^{2}\left\|E_{u}^{d, n-1}\right\|_{0}^{2} \\
& \left.+\Delta t \sum_{n=0}^{J}\left\|A u_{t}^{d, n-1}+\mathcal{O}(\Delta t)\right\|_{0}^{2}\left\|E_{\theta}^{d, n+1}\right\|_{0}^{2}\right\} .
\end{aligned}
$$

Finally, we present the optimal error estimate for pressure in $L^{\infty}\left(L^{2}\right)$ norm based on the results presented in Lemmas 18 and 19 .

Theorem 20. For all $J=0,1, \ldots,[T / \Delta t]-1$, under assumptions (A3)-(A7), one has

$$
\Delta t \sum_{n=0}^{J}\left\|p\left(t_{n+1}\right)-p^{d, n+1}\right\|_{0}^{2} \leq C \Delta t^{2} .
$$

Furthermore, if $u_{t} \in L^{\infty}(0, T ; X)$ and $\theta_{t} \in L^{\infty}(0, T ; W)$, then

$$
\left\|p\left(t_{n+1}\right)-p^{d, n+1}\right\|_{0} \leq C \Delta t .
$$

Proof. We can rewrite the first equation of problem (94) as

$$
\begin{aligned}
-\nabla E_{p}^{d, n+1}= & d_{t} E_{u}^{d, n+1}+\left(u\left(t_{n+1}\right) \cdot \nabla\right) u\left(t_{n+1}\right) \\
& -\left(u^{d, n} \cdot \nabla\right) u^{d, n+1}-v \Delta E_{u}^{d, n+1} \\
& -R_{u}^{n}+k v^{2} j E_{\theta}^{d, n}+k v^{2} j z_{\theta}^{n} .
\end{aligned}
$$

Take the inner product of (116) with an arbitrary $v \in X$ and use Poincare inequality to obtain

$$
\begin{aligned}
\left|k v^{2}\left(j z_{\theta}^{n}, v\right)\right| & \leq k v^{2}\left\|z_{\theta}^{n}\right\|_{0}\|v\|_{0} \\
& \leq C(k v)\left(\Delta t \int_{t_{n}}^{t_{n+1}}\left\|\theta_{t}\right\|_{0}^{2} d s\right)^{1 / 2}\|\nabla v\|_{0}, \\
\left|k v^{2}\left(j E_{\theta}^{n}, v\right)\right| & \leq k v^{2}\left\|E_{\theta}^{n}\right\|_{0}\|v\|_{0} \leq k v^{2}\left\|E_{\theta}^{n}\right\|_{0}\|\nabla v\|_{0} .
\end{aligned}
$$

For the nonlinear terms, use Lemma 3 to arrive at

$$
\begin{aligned}
& \left|b\left(E_{u}^{d, n}, u\left(t_{n+1}\right), v\right)\right| \leq C_{5}\left\|E_{u}^{d, n}\right\|_{0}\left\|A u\left(t_{n+1}\right)\right\|_{0}\|\nabla v\|_{0}, \\
& \left|b\left(u^{d, n}, E_{u}^{d, n+1}, v\right)\right| \leq C_{5}\left\|A u^{d, n}\right\|_{0}\left\|E_{u}^{d, n+1}\right\|_{0}\|\nabla v\|_{0}, \\
& \left|b\left(z_{u}^{n}, u\left(t_{n+1}\right), v\right)\right| \\
& \quad \leq C_{5}\left\|z_{u}^{n}\right\|_{0}\left\|A u\left(t_{n+1}\right)\right\|_{0}\|\nabla v\|_{0} \\
& \quad \leq C\left(\Delta t \int_{t_{n}}^{t_{n+1}}\left\|u_{t}\right\|_{0}^{2} d s\right)^{1 / 2}\left\|A u\left(t_{n+1}\right)\right\|_{0}\|\nabla v\|_{0} .
\end{aligned}
$$

Thus, thanks to (32), (86)-(88) and above inequalities, one finds

$$
\begin{aligned}
\left\|E_{p}^{d, n+1}\right\|_{0} \leq C\left(\beta^{-1}\right)\{ & \left\|d_{t} E_{u}^{d, n+1}\right\|_{0}+\nu\left\|\nabla E_{u}^{d, n+1}\right\|_{0} \\
& +\left\|A u^{d, n}\right\|_{0}\left\|E_{u}^{d, n+1}\right\|_{0} \\
& +\left\|A u\left(t_{n+1}\right)\right\|_{0}\left\|E_{u}^{d, n}\right\|_{0}+k \nu^{2}\left\|E_{\theta}^{d, n+1}\right\|_{0} \\
& +\Delta t\left(\int_{t_{n}}^{t_{n+1}} t\left\|u_{t t}\right\|_{0}^{2} d t\right)^{1 / 2} \\
& \left.+\left(\Delta t \int_{t_{n}}^{t_{n+1}}\left\|u_{t}\right\|_{0}^{2}+\left\|\theta_{t}\right\|_{0}^{2}\right)^{1 / 2}\right\} .
\end{aligned}
$$

Thanks to Lemma 1, we obtain the desired results. 
TABLE 1: The convergence performance and CPU time of the coupled algorithm (3.2) at time $T=1.0$ with varying time step $\Delta t$ but fixed mesh $h=1 / 32$.

\begin{tabular}{|c|c|c|c|c|c|c|}
\hline$\Delta t$ & $\left\|u-u^{n}\right\|_{0} /\|u\|_{0}$ & $\left\|\nabla\left(u-u^{n}\right)\right\|_{0} /\|\nabla u\|_{0}$ & $\left\|p-p^{n}\right\|_{0} /\|p\|_{0}$ & $\left\|\theta-\theta^{n}\right\|_{0} /\|\theta\|_{0}$ & $\left\|\nabla\left(\theta-\theta^{n}\right)\right\|_{0} /\|\nabla \theta\|_{0}$ & $\mathrm{CPU}(\mathrm{S})$ \\
\hline 0.1 & 0.000171956 & 0.0127757 & 0.00415879 & 0.000115897 & 0.0104146 & 174.829 \\
\hline 0.05 & 0.000162403 & 0.0127748 & 0.00416427 & 0.000107522 & 0.0104136 & 270.308 \\
\hline 0.025 & 0.00015784 & 0.0127745 & 0.00416706 & 0.000103686 & 0.0104134 & 433.188 \\
\hline 0.0125 & 0.000155623 & 0.0127744 & 0.0041685 & 0.000101859 & 0.0104133 & 864.545 \\
\hline 0.00625 & 0.000154529 & 0.0127744 & 0.00416949 & 0.000100969 & 0.0104133 & 1626.97 \\
\hline
\end{tabular}

TABLE 2: The convergence performance and CPU time of the decoupled algorithm (3.6) at time $T=1.0$ with varying time step $\Delta t$ but fixed mesh $h=1 / 32$.

\begin{tabular}{lccccc}
\hline$\Delta t$ & $\left\|u-u^{n}\right\|_{0} /\|u\|_{0}$ & $\left\|\nabla\left(u-u^{n}\right)\right\|_{0} /\|\nabla u\|_{0}$ & $\left\|p-p^{n}\right\|_{0} /\|p\|_{0}$ & $\left\|\theta-\theta^{n}\right\|_{0} /\|\theta\|_{0}$ & $\left\|\nabla\left(\theta-\theta^{n}\right)\right\|_{0} /\|\nabla \theta\|_{0}$ \\
\hline 0.1 & 0.000148351 & 0.0127747 & 0.00424037 & 0.000115877 & 0.0104146 \\
0.05 & 0.000150406 & 0.0127744 & 0.00419557 & 0.000107511 & 0.0104136 \\
0.025 & 0.00015181 & 0.0127744 & 0.00418087 & 0.000103673 & 0.0104134 \\
0.0125 & 0.000152594 & 0.0127744 & 0.0041747 & 0.00010185 & 0.0104133 \\
0.00625 & 0.000153011 & 0.0127744 & 0.00417207 & 0.000100964 & 0.0104133 \\
\hline
\end{tabular}

Squaring (119) and summing it from $n=0$ to $J$, with the results obtained in Lemmas 6,18 , and 19 , we obtain the desired result.

Furthermore, under the assumptions of $u_{t} \in L^{\infty}(0, T ; X)$ and $\theta_{t} \in L^{\infty}(0, T ; W),(119)$ can be transformed into

$$
\begin{aligned}
\left\|E_{p}^{d, n+1}\right\|_{0} \leq C\left(\beta^{-1}\right)\{ & \left\|d_{t} E_{u}^{d, n+1}\right\|_{0} \\
& +\nu\left\|\nabla E_{u}^{d, n+1}\right\|_{0}+\left\|A u^{d, n}\right\|_{0}\left\|E_{u}^{d, n+1}\right\|_{0} \\
& +\left\|A u\left(t_{n+1}\right)\right\|_{0}\left\|E_{u}^{d, n}\right\|_{0}+k v^{2}\left\|E_{\theta}^{d, n+1}\right\|_{0} \\
& +\Delta t\left(\int_{t_{n}}^{t_{n+1}} t\left\|u_{t t}\right\|_{0}^{2} d t\right)^{1 / 2} \\
& \left.+\Delta t\left(\left\|u_{t}\right\|_{0}^{2}+\left\|\theta_{t}\right\|_{0}^{2}\right)\right\} .
\end{aligned}
$$

With the help of Lemmas 6, 18, and 19, we obtain the optimal error estimate for pressure in $L^{\infty}\left(L^{2}\right)$ norm.

\section{Numerical Experiments}

In order to gain insights into the established convergence results in Sections 5 and 6, we present some numerical tests in this section. Our main interest is to verify and compare the performances of the coupled and decoupled algorithms (28) and (31). In all experiments, the time-dependent natural convection problem is defined on a convex domain $\Omega=$ $[0,1] \times[0,1]$. The mesh consists of triangular elements that are obtained by dividing $\Omega$ into subsquares of equal size and then drawing the diagonal in each subsquare. The model parameters $\nu, k$, and $\lambda$ are simply set to 1 . We fix the mesh size $h=1 / 32$ and use the MINI element which satisfies the discrete inf-sup condition to approximate the velocity $u$ and pressure $p$ and the linear polynomial to approximate the temperature $\theta$. The boundary and initial conditions and righthand side functions $f$ and $g$ are selected such that the exact solutions are given by

$$
\begin{aligned}
& u_{1}=10 x^{2}(x-1)^{2} y(y-1)(2 y-1) \cos (t) \\
& u_{2}=-10 x(x-1)(2 x-1) y^{2}(y-1)^{2} \cos (t) \\
& p=10(2 x-1)(2 y-1) \cos (t) \\
& \theta=10 x^{2}(x-1)^{2} y(y-1)(2 y-1) \cos (t) \\
& \quad-10 x(x-1)(2 x-1) y^{2}(y-1)^{2} \cos (t),
\end{aligned}
$$

where the components of $u$ are denoted by $\left(u_{1}, u_{2}\right)$ for convenience.

Firstly, we compare the errors and CPU times for the coupled and decoupled numerical schemes with varying time step $\Delta t$. From Tables 1 and 2, we can see that two kinds of numerical schemes almost get the same accuracy, but the decoupled scheme (31) spends much less CPU time than the coupled scheme (28). In other words, the decoupled scheme is comparable with the coupled scheme but cheaper and more efficient.

Secondly, we focus on examining the orders of convergence of the coupled and decoupled numerical schemes with respect to the time step. Following [6], we introduce a more accurate approach to examine the orders of convergence with respect to the time step $\Delta t$ due to the approximation errors $\mathcal{O}\left(\Delta t^{\gamma}\right)$. For example, assuming

$$
v^{\Delta t} \approx v\left(x, t_{n}\right)+C\left(x, t_{n}\right) \Delta t^{\gamma}
$$


TABLE 3: Convergence orders of the coupled algorithm (28) at time $T=1.0$ with varying time step $\Delta t$ but fixed mesh $h=1 / 32$.

\begin{tabular}{lccccc}
\hline$\Delta t$ & $\left\|u^{n, \Delta t}-u^{n, \Delta t / 2}\right\|_{0}$ & $\rho_{u, \Delta t, 0}$ & $\left\|u^{n, \Delta t}-u^{n, \Delta t / 2}\right\|_{1}$ & $\rho_{u, \Delta t, 1}$ & $\left\|p^{n, \Delta t}-p^{n, \Delta t / 2}\right\|_{0}$ \\
\hline 0.1 & $1.09819 e-005$ & 2.06348 & $7.97307 e-005$ & 2.06314 & $2.09931 e-005$ \\
0.05 & $5.32202 e-006$ & 2.04257 & $3.86453 e-005$ & 2.03837 & $1.04834 e-005$ \\
0.025 & $2.60556 e-006$ & 2.01799 & $1.89589 e-005$ & 1.91156 & $6.38507 e-006$ \\
0.0125 & $1.29116 e-006$ & & $9.91804 e-006$ & & $1.03158 e-005$ \\
\hline$\Delta t$ & $\left\|\theta^{n, \Delta t}-\theta^{n, \Delta t / 2}\right\|_{0}$ & $\rho_{\theta, \Delta t, 0}$ & $\left\|\theta^{n, \Delta t}-\theta^{n, \Delta t / 2}\right\|_{1}$ & $\rho_{\theta, \Delta t, 1}$ & 0.618958 \\
\hline 0.1 & $1.14663 e-005$ & 2.06739 & $8.10132 e-005$ & 2.06742 & \\
0.05 & $5.54628 e-006$ & 2.03617 & $3.91857 e-005$ & 2.03617 & \\
0.025 & $2.72388 e-006$ & 2.01728 & $1.92448 e-005$ & 2.01729 & \\
0.0125 & $1.35028 e-006$ & & $9.53992 e-006$ & & \\
\hline
\end{tabular}

TABLE 4: Convergence orders of the decoupled algorithm (31) at time $T=1.0$ with varying time step $\Delta t$ but fixed mesh $h=1 / 32$.

\begin{tabular}{lccccc}
\hline$\Delta t$ & $\left\|u^{d, n, \Delta t}-u^{d, n, \Delta t / 2}\right\|_{0}$ & $\rho_{u, \Delta t, 0}$ & $\left\|u^{d, n, \Delta t}-u^{d, n, \Delta t / 2}\right\|_{1}$ & $\rho_{u, \Delta t, 1}$ & $\left\|p^{d, n, \Delta t}-p^{d, n, \Delta t / 2}\right\|_{0}$ \\
\hline 0.1 & $4.44724 e-006$ & 1.72372 & $5.735 e-005$ & 2.42956 & 0.000276178 \\
0.05 & $2.58002 e-006$ & 1.88641 & $2.36051 e-005$ & 1.91411 & 0.000124308 \\
0.025 & $1.36769 e-006$ & 1.9327 & $1.23322 e-005$ & 1.93498 & $6.35925 e-005$ \\
0.0125 & $7.07654 e-007$ & & $6.37328 e-006$ & & $3.16876 e-005$ \\
\hline$\Delta t$ & $\left\|\theta^{d, n, \Delta t}-\theta^{d, n, \Delta t / 2}\right\|_{0}$ & $\rho_{\theta, \Delta t, 0}$ & $\left\|\theta^{d, n, \Delta t}-\theta^{d, n, \Delta t / 2}\right\|_{1}$ & $\rho_{\theta, \Delta t, 1}$ & 2.00686 \\
\hline 0.1 & $1.14672 e-005$ & 2.06806 & $8.10194 e-005$ & 2.06807 & \\
0.05 & $5.54493 e-006$ & 2.03505 & $3.91763 e-005$ & 2.03508 & \\
0.025 & $2.72472 e-006$ & 2.01706 & $1.92505 e-005$ & 2.01679 & \\
0.0125 & $1.35084 e-006$ & & $9.54514 e-006$ & & \\
\hline
\end{tabular}

thus we have

$$
\rho_{v, \Delta t, j}=\frac{\left\|v^{\Delta t}\left(x, t_{n}\right)-v^{\Delta t / 2}\left(x, t_{n}\right)\right\|_{j}}{\left\|v^{\Delta t / 2}\left(x, t_{n}\right)-v^{\Delta t / 4}\left(x, t_{n}\right)\right\|_{j}} \approx \frac{4^{\gamma}-2^{\gamma}}{2^{\gamma}-1} .
$$

Here, $v$ can take $u, p, \theta$ and $j$ can be 0 or 1 . While $\rho_{v, \Delta t, j}$ approach 4.0 or 2.0 , the convergence order will be 2.0 or 1.0 , respectively.

In Tables 3 and 4, we present the convergence orders with the fixed spacing $h=1 / 32$ and varying time steps $\Delta t=$ $0.1,0.05,0.025,0.0125$. From these results, we can see that the decoupled scheme almost gets the same accuracy with the coupled scheme. For the numerical solutions $u^{n}$ and $\theta^{n}$ of the coupled scheme (28), we can get the optimal orders of convergence; for the pressure $p^{n}$, the results are undesired. In contrast, the results in Table 4 strongly suggest that the orders of convergence in time are $\mathcal{O}(\Delta t)$, which implies that the error estimates for the $L^{2}$-norm and $H^{1}$-norm of $u^{d, n}, p^{d, n}$, and $\theta^{d, n}$ in the decoupled algorithm (31) are optimal. Our numerical results confirm the established theoretical analysis very well.

\section{Conflict of Interests}

The authors declare that there is no conflict of interests regarding the publication of this paper.

\section{Acknowledgments}

This work was supported by the NSF of China (no. 11301157), the Doctor Fund of Henan Polytechnic University (B2012098), the Natural Science Foundation of Education Department of Henan Province (no. 14A110008), and the Foundation of Distinguished Young's Scientists of Henan Polytechnic University (J2015-05).

\section{References}

[1] F. Brezzi and M. Fortin, Mixed and Hybrid Finite Element Methods, vol. 15 of Springer Series in Computational Mathematics, Springer, New York, NY, USA, 1991.

[2] S. M. Shen, "The finite element analysis of conduction-convection problem," Journal of Computational Mathematics, vol. 16, pp. 170-182, 1994 (Chinese).

[3] M. A. Christon, P. M. Gresho, and S. B. Sutton, "Computational predictability of time-dependent natural convection flows in enclosures (including a benchmark solution)," International Journal for Numerical Methods in Fluids, vol. 40, pp. 953-980, 2002, MIT Special Issue on Thermal Convection.

[4] A. Çıbık and S. Kaya, "A projection-based stabilized finite element method for steady-state natural convection problem," Journal of Mathematical Analysis and Applications, vol. 381, no. 2, pp. 469-484, 2011.

[5] M. Mu and J. Xu, "A two-grid method of a mixed Stokes-Darcy model for coupling fluid flow with porous media flow," SIAM Journal on Numerical Analysis, vol. 45, no. 5, pp. 1801-1813, 2007. 
[6] M. Mu and X. Zhu, "Decoupled schemes for a non-stationary mixed Stokes-Darcy model," Mathematics of Computation, vol. 79, no. 270, pp. 707-731, 2010.

[7] W. J. Layton, F. Schieweck, and I. Yotov, "Coupling fluid flow with porous media flow," SIAM Journal on Numerical Analysis, vol. 40, no. 6, pp. 2195-2218 (2003), 2002.

[8] W. Layton, H. Tran, and C. Trenchea, "Analysis of long time stability and errors of two partitioned methods for uncoupling evolutionary groundwater-surface water flows," SIAM Journal on Numerical Analysis, vol. 51, no. 1, pp. 248-272, 2013.

[9] T. Zhang and J. Y. Yuan, "Two novel decoupling finite element algorithms for the steady Stokes-Darcy model based on two grid discretization," Discrete and Continuous Dynamical Systems, Series B, vol. 19, pp. 849-865, 2014.

[10] T. Zhang, X. Zhao, and P. Z. Huang, "Decoupled two level finite element methods for the steady natural convection problem," Numerical Algorithms, 2014.

[11] Y. He, "Two-level method based on finite element and CrankNicolson extrapolation for the time-dependent NAVier-Stokes equations," SIAM Journal on Numerical Analysis, vol. 41, no. 4, pp. 1263-1285, 2003.

[12] J. Heywood and R. Rannacher, "Finite element approximation of the nonstationary Navier-Stokes problem I; regularity of solutions and second-order error estimates for spatial discretization," SIAM Journal on Numerical Analysis, vol. 19, pp. 275-311, 1982.

[13] P. G. Ciarlet, The Finite Element Method for Elliptic Problems, North-Holland, Amsterdam, The Netherlands, 1978.

[14] R. Temam, Navier-Stokes Equation: Theory and Numerical Analysis, North-Holland, Amsterdam, The Netherlands, 3rd edition, 1984.

[15] J. Blasco and R. Codina, "Error estimates for an operatorsplitting method for incompressible flows," Applied Numerical Mathematics, vol. 51, no. 1, pp. 1-17, 2004.

[16] J. Blasco, R. Codina, and A. Huerta, "A fractional-step method for the incompressible Navier-Stokes equations related to a predictor-multicorrector algorithm," International Journal for Numerical Methods in Fluids, vol. 28, no. 10, pp. 1391-1419, 1998.

[17] F. Guillén-González and M. V. Redondo-Neble, "New error estimates for a viscosity-splitting scheme in time for the threedimensional Navier-Stokes equations," IMA Journal of Numerical Analysis, vol. 31, no. 2, pp. 556-579, 2011.

[18] J. Shen, "On error estimates of projection methods for NavierStokes equations: first-order schemes," SIAM Journal on Numerical Analysis, vol. 29, no. 1, pp. 57-77, 1992.

[19] J. Shen, "Remarks on the pressure error estimates for the projection methods," Numerische Mathematik, vol. 67, no. 4, pp. 513-520, 1994.

[20] Y. He, "The Euler implicit/explicit scheme for the 2D timedependent Navier-Stokes equations with smooth or nonsmooth initial data," Mathematics of Computation, vol. 77, no. 264, pp. 2097-2124, 2008.

[21] Z. D. Luo, The Bases and Applications of Mixed Finite Element Methods, Chinese Science Press, Beijing, China, 2006.

[22] Y. He, "Stability and error analysis for a spectral Galerkin method for the Navier-Stokes equations with $H^{2}$ or $H^{1}$ initial data," Numerical Methods for Partial Differential Equations, vol. 21, no. 5, pp. 875-904, 2005.

[23] Y. He and J. Li, "A penalty finite element method based on the Euler implicit/explicit scheme for the time-dependent Navier-Stokes equations," Journal of Computational and Applied Mathematics, vol. 235, no. 3, pp. 708-725, 2010.
[24] T. Zhang, D. Pedro, and J. Y. Yuan, "A large time stepping viscosity-splitting finite element method for the viscoelastic flow problem," Advances in Computational Mathematics, 2014. 


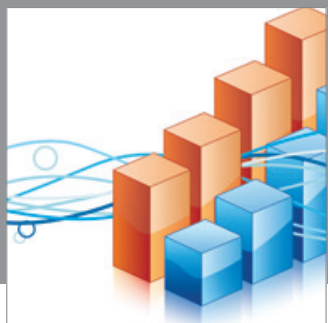

Advances in

Operations Research

mansans

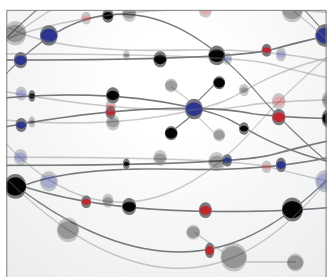

The Scientific World Journal
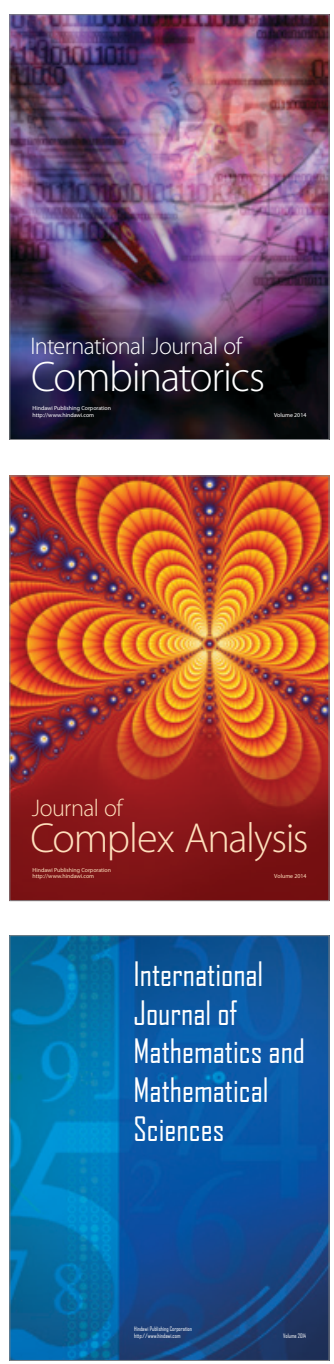
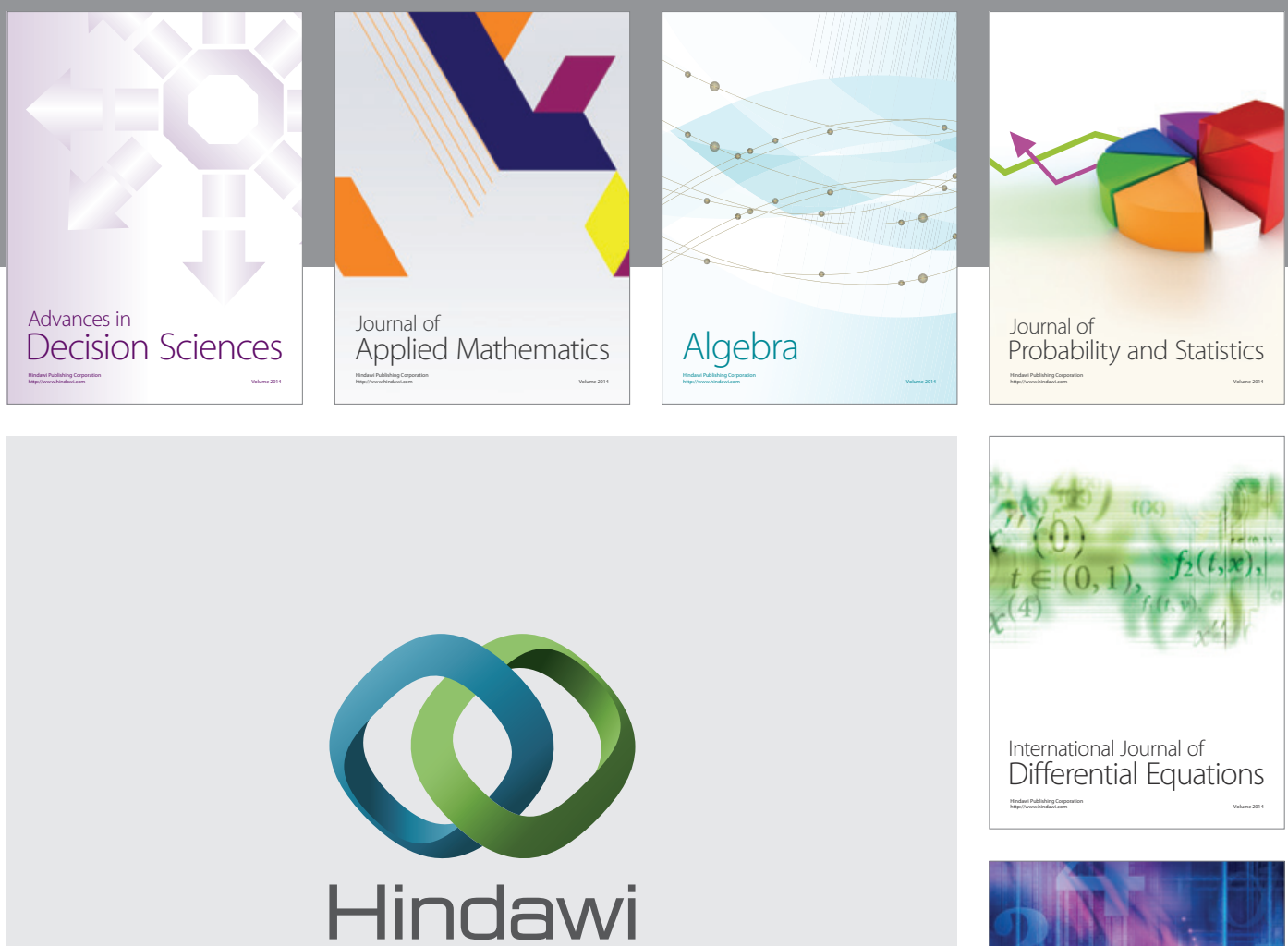

Submit your manuscripts at http://www.hindawi.com
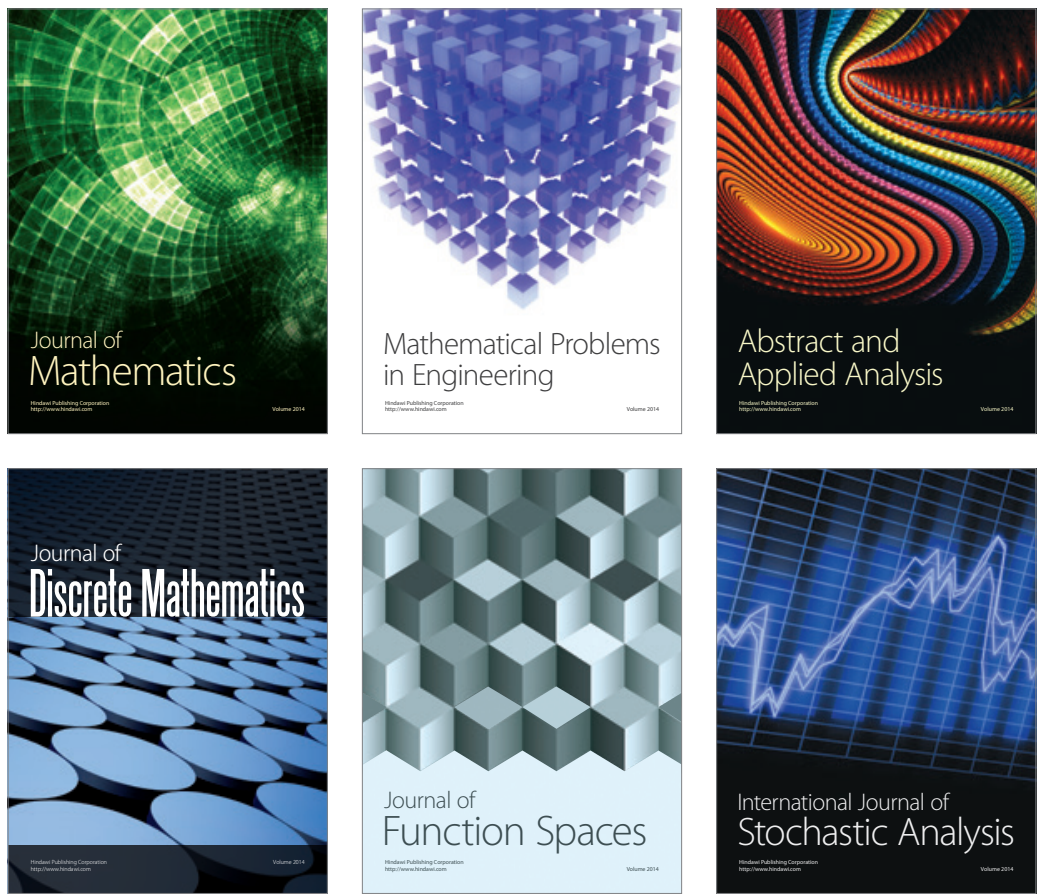

Journal of

Function Spaces

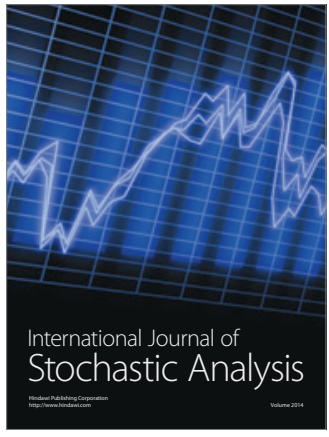

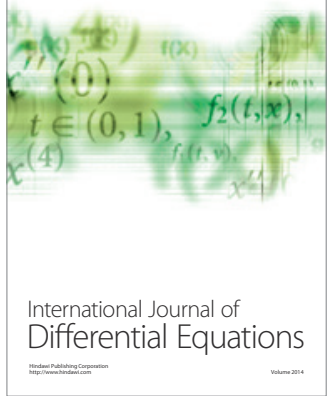
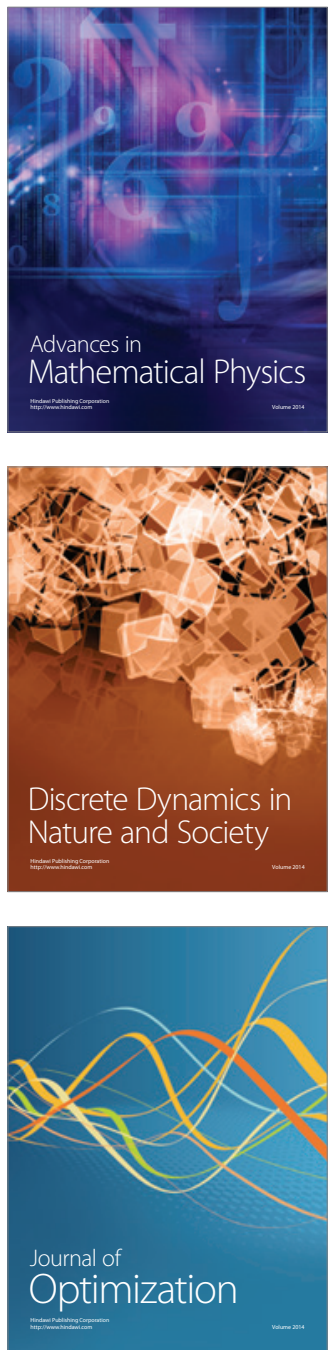